\title{
A Rapid Method of Evaluating the Regulator and Class Number of a Pure Cubic Field
}

\author{
By H. C. Williams*, G. W. Dueck and B. K. Schmid
}

\begin{abstract}
Let $\mathcal{K}=\mathscr{2}(\theta)$ be the algebraic number field formed by adjoining $\theta$ to the rationals 2. Let $R$ and $h$ be, respectively, the regulator and class number of $\mathscr{K}$. Shanks has described a method of evaluating $R$ for $\mathcal{2}(\sqrt{D})$, where $D$ is a positive integer. His technique improved the speed of the usual continued fraction algorithm for finding $R$ by allowing one to proceed almost directly from the $n$th to the $m$ th step, where $m$ is approximately $2 n$, in the continued fraction expansion of $\sqrt{D}$. This paper shows how Shanks' idea can be extended to the Voronoi algorithm, which is used to find $R$ in cubic fields of negative discriminant. It also discusses at length an algorithm for finding $R$ and $h$ for pure cubic fields $2(\sqrt[3]{D}), D$ an integer. Under a certain generalized Riemann Hypothesis the ideas developed here will provide a new method which will find $R$ and $h$ in $O\left(D^{2 / 5+\varepsilon}\right)$ operations. When $h$ is small, this is an improvement over the $O(D / h)$ operations required by Voronoi's algorithm to find $R$. For example, with $D=200171999$, it required only 5 minutes for an AMDAHL $470 / V 7$ computer to find that $R=518594546.969083$ and $h=1$. This same calculation would require about 8 days of computer time if it used only the standard Voronoi algorithm.
\end{abstract}

1. Introduction. Let $\mathcal{Z}$ be the set of rational integers, and let $\delta$ be the real root of the irreducible cubic equation

$$
x^{3}-B x^{2}+C x-D=0
$$

where $B, C, D \in \mathscr{Z}$ and the discriminant $\Delta$ of $(1.1)$ is negative. Let $\mathcal{K}=2(\delta)$ be the cubic field formed by adjoining $\delta$ to the rationals 2 , and let $2[\delta]$ be the ring of algebraic integers in $\mathcal{K}$. It is well known (see, for example, Delone and Faddeev $[6$, pp. 111-112]) that $2(\delta)$ has an integral basis $\{1, \mu, \nu\}$, where

$$
\begin{gathered}
\mu=\left(m_{1}+m_{2} \delta+m_{3} \delta^{2}\right) / \sigma, \quad \nu=\left(n_{1}+n_{2} \delta+n_{3} \delta^{2}\right) / \sigma, \\
\sigma, m_{1}, m_{2}, m_{3}, n_{1}, n_{2}, n_{3} \in \mathscr{Z}, \quad \sigma>0, \quad \text { and } \\
\operatorname{gcd}\left(m_{1}, m_{2}, m_{3}, n_{1}, n_{2}, n_{3}, \sigma\right)=1 .
\end{gathered}
$$

Also, if $e=m_{2} n_{3}-m_{3} n_{2}$, then $e \mid \sigma$.

If $\omega \in \mathcal{K}$, we denote its conjugates by $\omega^{\prime}$ and $\omega^{\prime \prime}$. We also write the norm of $\omega$ as $N(\omega)$ and define it as $N(\omega)=\omega \omega^{\prime} \omega^{\prime \prime}$. From the simple fact that

$$
N(\omega)\left|\begin{array}{ccc}
1 & 1 & 1 \\
\delta & \delta^{\prime} & \delta^{\prime \prime} \\
\delta^{2} & \delta^{\prime 2} & \delta^{\prime \prime 2}
\end{array}\right|=\left|\begin{array}{ccc}
\omega & \omega^{\prime} & \omega^{\prime \prime} \\
\delta \omega & \delta^{\prime} \omega^{\prime} & \delta^{\prime \prime} \omega^{\prime \prime} \\
\delta^{2} \omega & \delta^{\prime 2} \omega^{\prime} & \delta^{\prime \prime 2} \omega^{\prime \prime}
\end{array}\right|
$$

Received November 3, 1982.

1980 Mathematics Subject Classification. Primary 12A35, 12A45, 12 A50.

*Research supported by NSERC grant number A7649. 
we can deduce that if $\omega=\left(q_{1}+q_{2} \delta+q_{3} \delta^{2}\right) / q_{4}, q_{i} \in \stackrel{(}{=}(i=1,2,3,4)$, then

$$
q_{4}^{3} N(\omega)=\left|\begin{array}{ccc}
q_{1} & q_{2} & q_{3} \\
D q_{3} & q_{1}-q_{3} C & q_{2}+B q_{3} \\
D q_{2}+D B q_{3} & (D-B C) q_{3}-q_{2} C & q_{1}+B q_{2}+\left(B^{2}-C\right) q_{3}
\end{array}\right|
$$

If $\varepsilon \in \stackrel{Q}{\sim}[\delta]$ and $|N(\varepsilon)|=1$, we say that $\varepsilon$ is a unit of $h$. In the case of the complex cubic fields which we are discussing, there exists $\varepsilon_{0} \in{ }^{()}[\delta]$ such that $\varepsilon_{0}>1$ and, if $\varepsilon$ is any unit of $t h$, there exists some $n \in \stackrel{\sim}{=}$ for which $\varepsilon= \pm \varepsilon_{0}^{n}$. We call $\varepsilon_{0}$ the fundamental unit of $h$, and we call $R=\log \varepsilon_{0}$ the regulator of $h$. We denote the class number of th by $h$.

When $B=C=0$ we say that $h=\sqrt{2}(\sqrt[3]{D})$ is a pure cubic field with radicand $D$. We may assume with no loss of generality that $D=g_{1} g_{2}^{2}$ and $g_{1} g_{2}$ is square free. When $D Z \pm 1(\bmod 9)$, put $\iota=0$; in this case values of $m_{i}, n_{i}, \sigma$ in $(1.2)$ are $\sigma=g_{2}$, $m_{1}=0, m_{2}=g_{2}, m_{3}=0, n_{1}=0, n_{2}=0, n_{3}=1$. When $D \equiv \pm 1(\bmod 9)$, put $\iota=1$; in this case values of $m_{i}, n_{i}, \sigma$ in (1.2) are $\sigma=3 g_{2}, m_{1}=0, m_{2}=3 g_{2}$, $m_{3}=0, n_{1}=g_{2}^{2}, n_{2}= \pm g_{2}^{2}, n_{3}=1$. Put $J=3^{1-\imath} g_{1} g_{2}$.

Several previous papers (see Williams [16] for references) have dealt empirically with the problem of the distribution of those values of $D$ for which $h$ of $2(\sqrt[3]{D})$ has value 1 . It was very difficult to continue the computations for $D>2 \times 10^{5}$, because the values of the regulators, which were needed in the determination of $h$, were taking so long to compute. Thus, in order to continue these calculations, a faster method of evaluating $R$ is needed. In [12] Shanks discussed a rapid technique for finding the regulator of a real quadratic field $\mathcal{Q}(\sqrt{D})$ when $D$ is large. His technique improves the speed of the continued fraction scheme by allowing one to proceed almost directly from the $n$th step to the $m$ th step in the continued fraction, where $m \simeq 2 n .^{* *}$ Recently Lenstra [9] and Schoof [11] have given another version of the ideas in [12]. Both Shanks (in [19] and [13]) and Lenstra (in [9]) pointed out that it should be possible to extend Shanks' ideas to the cubic case $Q(\delta)$. In this paper we describe a means by which this can be done in the case of a pure cubic field. Instead of using the usual continued fraction that Shanks discussed, we will centre our discussion around the continued fraction algorithm of Voronoi [15]. While we have tended to restrict our discussion to the pure cubic case with $D>10^{5}$, the main ideas presented here can be extended to any cubic field with negative discriminant. Indeed, Section 2 and much of Sections 3, 4 and 7 are quite general in this respect.

In Table 1 below, we summarize some of the notation used in this paper.

TABLE 1

\begin{tabular}{ll}
\hline Symbol & Description \\
\hline$Z$ & The set of rational integers. \\
\hline$B, C, D, \delta$ & $\delta$ is the real zero of an irreducible polynomial \\
& $x^{3}-B x^{2}+C x-D$ with integer coefficients and \\
& negative discriminant.
\end{tabular}

** We use the symbol $\simeq$ to denote "approximately equal to". 


\section{TABLE 1 (continued)}

\begin{tabular}{|c|c|}
\hline$h=\stackrel{(0)}{-}(\delta)$, & $\begin{array}{l}\text { The cubic field formed by adjoining } \delta \text { to the rationals } \\
\text { (2). }\end{array}$ \\
\hline$\because[\delta]$ & The ring of algebraic integers in $\mathcal{2}(\delta)$ \\
\hline$e, \sigma$ & $\begin{array}{l}\text { For any basis }\{1, \mu, \nu\} \text { of } \mathcal{Q}[\delta], \text { we have } \\
\mu=\left(m_{1}+m_{2} \delta+m_{3} \delta^{2}\right) / \sigma, \nu=\left(n_{1}+n_{2} \delta+n_{3} \delta^{2}\right) / \sigma, \\
\text { where } m_{1}, m_{2}, m_{3}, n_{1}, n_{2}, n_{3}, \sigma \in \stackrel{\sigma g}{2}, \sigma>0, \\
\operatorname{gcd}\left(m_{1}, m_{2}, m_{3}, n_{1}, n_{2}, n_{3}, \sigma\right)=1 \text {. The values of } \sigma \\
\text { and } e=m_{2} n_{3}-n_{2} m_{3} \text { are independent of the choice of } \\
\text { basis. }\end{array}$ \\
\hline$\varepsilon_{0}$ & The fundamental unit of $\gamma$. \\
\hline$R=\log \varepsilon_{0}$ & The regulator of $h$. \\
\hline$h$ & The class number of $h$. \\
\hline$g_{1}, g_{2}, \iota, J$ & 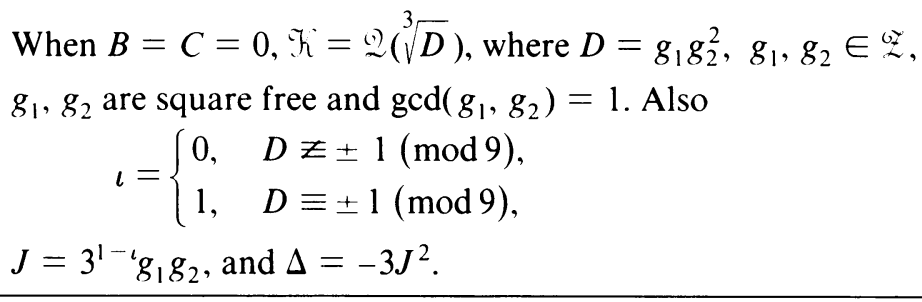 \\
\hline$G L_{n}(\stackrel{q Z}{=})$ & $\begin{array}{l}\text { The group of all } n \times n \text { matrices with rational integer } \\
\text { entries and determinant equal to } \pm 1 \text {. }\end{array}$ \\
\hline $\begin{array}{l}\omega, \omega^{\prime}, \omega^{\prime \prime} \\
N(\omega)\end{array}$ & $\begin{array}{l}\text { If } \omega \notin \mathcal{Q} \text {, we say that } \omega^{\prime}, \omega^{\prime \prime} \text { are the two conjugates } \\
\text { of } \omega . N(\omega)=\omega \omega^{\prime} \omega^{\prime \prime} . \text { If } \omega \in \mathcal{Q} \text {, then } N(\omega)=\omega^{3} \text {. }\end{array}$ \\
\hline$\Omega$ & $\begin{array}{l}\Omega=\left(\omega,\left(\omega^{\prime}-\omega^{\prime \prime}\right) / 2 i,\left(\omega^{\prime}+\omega^{\prime \prime}\right) / 2\right) \text {, where } i^{2}+1=0 \text {. This } \\
\text { is equivalent to writing } \Omega \approx \omega \text { or } \omega \approx \Omega \text {. }\end{array}$ \\
\hline $\begin{array}{l}\Re=\left\langle\lambda_{1}, \lambda_{2}, \lambda_{3}\right\rangle \\
\gamma(\gamma \in \mathcal{R})\end{array}$ & $\begin{array}{l}\text { For } \lambda_{1}, \lambda_{2}, \lambda_{3} \in \mathscr{K} \text {, we have } \\
\Re=\left\langle\lambda_{1}, \lambda_{2}, \lambda_{3}\right\rangle=\left\{\Omega \mid \Omega \approx \omega=l_{1} \lambda_{1}+l_{2} \lambda_{2}+l_{3} \lambda_{3},\right. \\
\left.l_{1}, l_{2}, l_{3} \in \mathscr{Z}\right\} . \mathscr{R} \text { has a basis }\left\{\lambda_{1}, \lambda_{2}, \lambda_{3}\right\} . \\
\gamma \mathscr{R}=\left\langle\gamma \lambda_{1}, \gamma \lambda_{2}, \gamma \lambda_{3}\right\rangle . \text { If } \Re=\langle 1, \mu, \nu\rangle, \\
\mu, \nu \in \mathscr{K}, \text { we say that } \mathscr{R} \text { is a 1-lattice }\end{array}$ \\
\hline$f, \rho$ & $\begin{array}{l}\text { If } \mathcal{R} \text { is a 1-lattice }\langle 1, \mu, \nu\rangle \text { with } \\
\mu=\left(m_{1}+m_{2} \delta+m_{3} \delta^{2}\right) / \rho, \nu=\left(n_{1}+n_{2} \delta+n_{3} \delta^{2}\right) / \rho, \\
\text { where } m_{1}, m_{2}, m_{3}, n_{1}, n_{2}, n_{3}, \rho \in \mathscr{Z}, \rho>0 \text {, } \\
\operatorname{gcd}\left(m_{1}, m_{2}, m_{3}, n_{1}, n_{2}, n_{3}, \rho\right)=1, \text { then the } \\
\text { values of } \rho \text { and } f=m_{2} n_{3}-m_{3} n_{2} \text { are independent } \\
\text { of the basis selected for } \mathcal{R} \text {. }\end{array}$ \\
\hline$e, e_{1}$ & $\begin{array}{l}\varrho=\left\{(x, y, z) \mid y^{2}+z^{2} \leqslant 1,(x, y, z) \in \mathcal{E}_{3}\right\} \\
\bigodot_{1}=\left\{(x, y, z)|| x \mid \leqslant 1, y^{2}+z^{2} \leqslant 1,(x, y, z) \in \mathcal{E}_{3}\right\} \\
\text { here } \varepsilon_{3} \text { is Euclidean 3-space. }\end{array}$ \\
\hline$\theta_{g}, \Theta_{g}$ & $\begin{array}{l}\Theta_{g}\left(\approx \theta_{g}\right) \text { is the relative minimum adjacent to }(1,0,1) \\
\text { in a reduced lattice } R .\end{array}$ \\
\hline
\end{tabular}


TABLE 1 (continued)

\begin{tabular}{|c|c|}
\hline$Q_{n}, e_{n}, \sigma_{n}, \theta_{g}^{(n)}$ & $\begin{array}{l}\mathscr{R}_{1}=\langle 1, \bar{\mu}, \bar{\nu}\rangle \text {, where }\{1, \bar{\mu}, \bar{\nu}\} \text { is any basis of } \\
\mathcal{Q}[\delta] . \theta_{g}^{(n)} \text { is } \theta_{g}, \sigma_{n} \text { is } \rho \text { and } e_{n} \text { is } f \text { for } \mathscr{R}_{n} \text {, } \\
\text { where } \\
\qquad \mathscr{Q}_{j+1}=\left(1 / \theta_{g}^{(j)}\right) \mathscr{G}_{j}(j=1,2,3, \ldots) .\end{array}$ \\
\hline$R_{n}, \theta_{n}$ & $\theta_{n}=\prod_{i=1}^{n-1} \theta_{g}^{(i)}, R_{n}=\log \theta_{n}$ \\
\hline$G R \sim \delta$ & $\mathscr{R} \sim \delta$ if $R$ and $\delta$ are 1-lattices and $\mathscr{R}=\gamma \delta, \gamma \in \mathcal{h}$. \\
\hline$F(Q), E$ & $\begin{array}{l}F(Q)=\prod_{q \leqslant Q} f(q), \text { where the product is taken over all } \\
\text { primes } q<Q \text { where } q \equiv 1(\bmod 3)(\text { see Section } 3) \text {. } \\
E=\sqrt{3} c_{2} J F(Q) / 2 \pi .\end{array}$ \\
\hline
\end{tabular}

$c, d_{i}, d_{i}^{\prime}, d_{i}^{\prime \prime} \quad c$ is a solution of

$$
\left\{\begin{array}{l}
x^{2} \equiv D\left(\bmod \sigma^{2}\right) \\
3 x^{2} \equiv 0(\bmod \sigma)
\end{array}\right.
$$

If $p$ is a prime, $d_{i}\left(d_{i}^{\prime}, d_{i}^{\prime \prime}\right)$ is (are) solution(s) of

$$
\left\{\begin{array}{l}
x^{3} \equiv D\left(\bmod \sigma^{2} p^{i}\right) \\
x \equiv c(\bmod \sigma)
\end{array}\right.
$$

such that none of $d_{i}, d_{i}^{\prime}, d_{i}^{\prime \prime}$ satisfies

\begin{tabular}{|c|c|}
\hline $\begin{array}{l}P, P^{\prime}, P^{\prime \prime} \\
r, s, s^{\prime}, L(a)\end{array}$ & $\begin{array}{l}\text { If a is any ideal of } \mathcal{2}[\delta](\delta=\sqrt[3]{D}) \text {, and a has no } \\
\text { rational integer divisor, then a has a basis of the form } \\
\left\{P, P^{\prime}(r+\delta), P^{\prime \prime}\left(s+s^{\prime} \delta+\delta^{2}\right) / \sigma\right\} \text {, where } P, P^{\prime}, P^{\prime \prime} \\
r, s, s^{\prime} \in \mathcal{Z}, P^{\prime} P^{\prime \prime}\left|P, P^{\prime \prime}\right| \sigma \text { and }(5.5) \text { holds. } L(a)=P \text {. }\end{array}$ \\
\hline$m, n, n^{\prime}$ & $\begin{array}{l}\text { A canonical basis of } \mathscr{R}\left(\sim \mathcal{G}_{1}\right) \text { has the form } \\
\left\{1,|f|(m+\delta) / \rho,\left(n+n^{\prime} \delta+\delta^{2}\right) / \rho\right\}, \text { where } \\
m, n, n^{\prime} \in Z \text {. Also, if a above is the ideal to } \\
\text { to which } \Re \text { corresponds, then } \rho=\sigma P / P^{\prime \prime}, \\
f=\sigma P^{\prime} / P^{\prime \prime}, P^{\prime \prime}=\sigma / \operatorname{gcd}(\sigma, f), m \equiv r(\bmod \rho / f), \\
n^{\prime} \equiv s(\bmod f), n \equiv s+r\left(n^{\prime}-s\right)(\bmod \rho) .\end{array}$ \\
\hline $\begin{array}{l}\omega_{p} \\
\zeta_{\omega}\end{array}$ & $\begin{array}{l}\text { If } \Omega \in \mathscr{R} \text { and } \Omega \approx \omega, \text { then } \xi_{\omega}=\left(2 \omega-\omega^{\prime}-\omega^{\prime \prime}\right) / 2, \\
\eta_{\omega}=\left(\omega^{\prime}-\omega^{\prime \prime}\right) / 2 i, \zeta_{\omega}=\left(\omega^{\prime}+\omega^{\prime \prime}\right) / 2 \text {. When } \\
\mathcal{K}=2(\sqrt[3]{D}) \text { and } \omega=\left(q_{1}+q_{2} \delta+q_{3} \delta^{2}\right) / \rho \\
q_{1}, q_{2}, q_{3} \in \mathcal{Z} \text {, then } \xi_{\omega}=3 \delta\left(q_{2}+q_{3} \delta\right) / 2 \rho, \\
\eta_{\omega}=\sqrt{3} \delta\left(q_{2}-q_{3} \delta\right) / 2 \rho, \zeta_{\omega}=\left(2 q_{1}-\delta q_{2}-\delta^{2} q_{3}\right) / 2 \rho\end{array}$ \\
\hline$\Gamma_{g}\left(\approx \gamma_{g}\right), \gamma_{h}$ & $\begin{array}{l}\Gamma_{g} \text { is a point of a 1-lattice } R\left(\sim \mathscr{R}_{1}\right) \text { such that } \\
\Gamma_{g} \in \mathcal{C}_{1} \text { and }\left|\gamma_{g}\right| \neq 1 \text {. Further, } \gamma_{h} \text { is any element } \\
\text { of } \Re \text { such that } R=\left\langle 1, \gamma_{g}, \gamma_{h}\right\rangle \text {. }\end{array}$ \\
\hline$\beta$ & $\beta=3.002 \delta^{2}$ \\
\hline
\end{tabular}

$x^{3} \equiv D\left(\bmod \sigma^{2} p^{i+1}\right)$. 


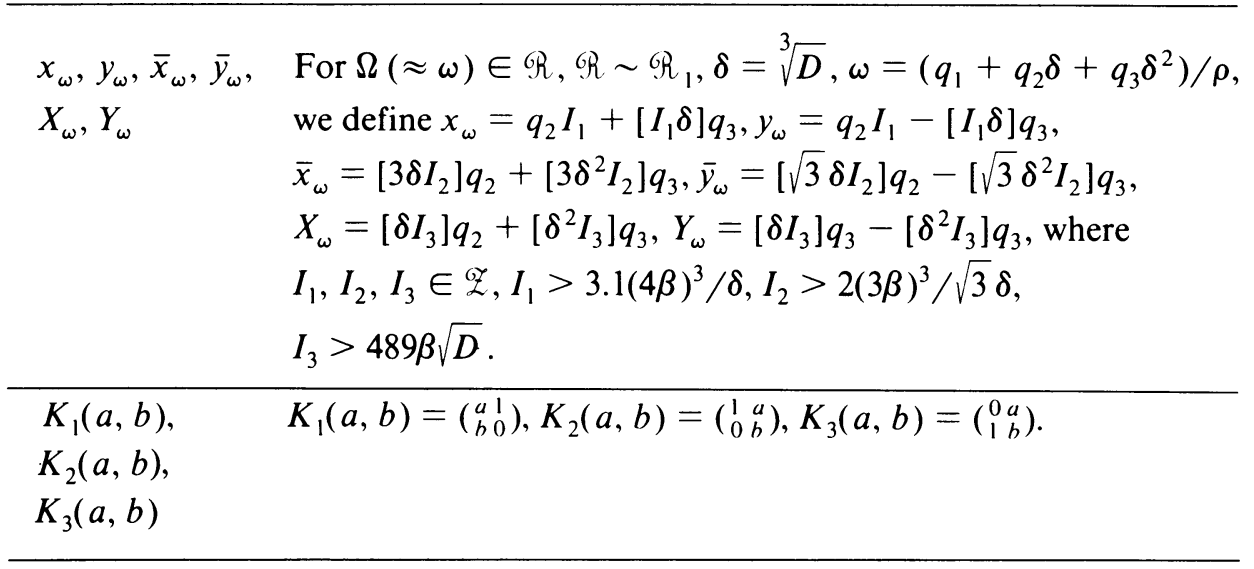

2. Ideals and Lattices. In this section, we give a brief discussion of some of the elementary properties of the ideals of $\mathcal{2}[\delta]$ and the lattices over $\mathcal{Z}(\delta)$. For a more detailed description of these ideas we refer the reader to [6].

Let $\lambda_{1}, \lambda_{2}, \lambda_{3} \in \mathcal{2}(\delta)$ such that

$$
\sum_{i=1}^{3} l_{i} \lambda_{i}=0 \quad\left(l_{1}, l_{2}, l_{3} \in Z\right)
$$

if and only if $l_{1}=l_{2}=l_{3}=0$. We say that $\mathcal{C}=\left\{\left(\omega, \omega^{\prime}, \omega^{\prime \prime}\right) \mid \omega=l_{1} \lambda_{1}+l_{2} \lambda_{2}+\right.$ $\left.l_{3} \lambda_{3} ; l_{1}, l_{2}, l_{3} \in \mathcal{Z}\right\}$ is a lattice over $\mathcal{Q}(\delta)$ with basis $\left\{\lambda_{1}, \lambda_{2}, \lambda_{3}\right\}$. Since we are dealing here with cubic fields with negative discriminant, we see that $\omega^{\prime}$ and $\omega^{\prime \prime}$ are complex. In order to work in real lattices, we use

$$
\begin{aligned}
\mathscr{R}=\left\{\Omega=\left(\omega,\left(\omega^{\prime}-\omega^{\prime \prime}\right) / 2 i,\left(\omega^{\prime}+\omega^{\prime \prime}\right) / 2\right) \mid\right. \\
\left.\omega=l_{1} \lambda_{1}+l_{2} \lambda_{2}+l_{3} \lambda_{3} ; l_{1}, l_{2}, l_{3} \in Z\right\},
\end{aligned}
$$

where $i$ is a fixed zero of $x^{2}+1$. Since $\Omega$ is uniquely determined by $\omega$, we often identify $\Omega$ with $\omega$ and write $\Omega \approx \omega$ or $\omega \approx \Omega$. $R$ is completely characterized by $\lambda_{1}, \lambda_{2}, \lambda_{3}$. We say that $\left\{\lambda_{1}, \lambda_{2}, \lambda_{3}\right\}$ is a basis of $R$, and we write $R=\left\langle\lambda_{1}, \lambda_{2}, \lambda_{3}\right\rangle$. Throughout this paper, we shall be restricting our attention to lattices of type $\Re$ over $\mathcal{Q}(\delta)$ which have a basis of the form $\left\{1, \lambda_{2}, \lambda_{3}\right\}$. We will call such a lattice a 1-lattice. We also note [6, p. 274] that if $\Omega(\approx \omega), \Phi(\approx \phi) \in \Re$ such that $\omega^{\prime} \omega^{\prime \prime}=\phi^{\prime} \phi^{\prime \prime}$, then $\omega= \pm \phi$. Further,

$$
\left|\omega^{\prime}\right|^{2}=\left|\omega^{\prime \prime}\right|^{2}=\omega^{\prime} \omega^{\prime \prime}=\left(\frac{\omega^{\prime}-\omega^{\prime \prime}}{2 i}\right)^{2}+\left(\frac{\omega^{\prime}+\omega^{\prime \prime}}{2}\right)^{2}
$$

Let $G L_{n}(\mathscr{Z})$ be the group of all $n \times n$ matrices $T$ such that $T$ has entries from $\mathscr{Z}$ only and $|T|= \pm 1$. If $\Re=\left\langle\lambda_{1}, \lambda_{2}, \lambda_{3}\right\rangle$ and $\Re^{*}=\left\langle\mu_{1}, \mu_{2}, \mu_{3}\right\rangle$, then $\Re=\Re^{*}$ if and only if

$$
\left(\begin{array}{l}
\mu_{1} \\
\mu_{2} \\
\mu_{3}
\end{array}\right)=T\left(\begin{array}{l}
\lambda_{1} \\
\lambda_{2} \\
\lambda_{3}
\end{array}\right)
$$




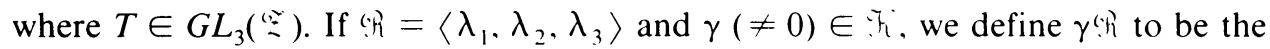
lattice $\left\langle\gamma \lambda_{1}, \gamma \lambda_{2}, \gamma \lambda_{3}\right\rangle$. If

$$
\gamma\left(\begin{array}{l}
\lambda_{1} \\
\lambda_{2} \\
\lambda_{3}
\end{array}\right)=T\left(\begin{array}{c}
1 \\
\mu_{1} \\
\mu_{2}
\end{array}\right)
$$

where $T \in G L_{3}(\stackrel{*}{=})$, then $\Re^{*}=\left\langle\gamma \lambda_{1}, \gamma \lambda_{2}, \gamma \lambda_{3}\right\rangle$ is a l-lattice. If $\left(R\right.$ and $R^{*}$ are both 1-lattices and $R^{*}=\gamma\left(R\right.$, we say that $h_{\text {nd }} h^{*}$ are similar and write this as $\left(h \sim \Re^{*}\right.$. Since both $h$ and $h^{*}$ are 1-lattices, this relationship is clearly an equivalence relationship.

Let $a \neq 0$ be any ideal of $\stackrel{0}{\sim}[\delta]$; then a can be written as

$$
\begin{aligned}
a & =\left[\kappa_{1}, \kappa_{2}, \kappa_{3}, \ldots, \kappa_{n}\right] \\
& =\left\{\sum_{i=1}^{n} \eta_{i} \kappa_{i} \mid \eta_{i} \in \stackrel{0}{-}[\delta], i=1,2,3, \ldots, n\right\},
\end{aligned}
$$

where the $\kappa_{i}$ 's are generators of a and $\kappa_{i} \in \mathcal{V}[\delta](i=1,2,3, \ldots, n)$. Also there exist $\nu_{1}, \nu_{2}, \nu_{3} \in \stackrel{?}{-}[\delta]$ such that

$$
a=\left\{\sum_{i=1}^{3} l_{i} \nu_{i} \mid l_{1}, l_{2}, l_{3} \in \stackrel{\Xi}{=}\right\} .
$$

This set $\left\{\nu_{1}, \nu_{2}, \nu_{3}\right\}$ is said to be a basis of a. If a has another basis $\left\{\tau_{1}, \tau_{2}, \tau_{3}\right\}$, then

$$
\left(\begin{array}{l}
\tau_{1} \\
\tau_{2} \\
\tau_{3}
\end{array}\right)=T\left(\begin{array}{l}
\nu_{1} \\
\nu_{2} \\
\nu_{3}
\end{array}\right)
$$

where $T \in G L_{3}(\stackrel{\Xi}{\mp})$. Since $\nu_{i}, \tau_{i} \in \mathcal{Q}[\delta](i=1,2,3)$, by (1.2) we must have

$$
\sigma\left(\begin{array}{l}
\nu_{1} \\
\nu_{2} \\
\nu_{3}
\end{array}\right)=M_{1}\left(\begin{array}{c}
1 \\
\delta \\
\delta^{2}
\end{array}\right), \quad \sigma\left(\begin{array}{c}
\tau_{1} \\
\tau_{2} \\
\tau_{3}
\end{array}\right)=M_{2}\left(\begin{array}{c}
1 \\
\delta \\
\delta^{2}
\end{array}\right),
$$

where $M_{1}$ and $M_{2}$ are $3 \times 3$ matrices with rational integer entries. Since $1, \delta, \delta^{2}$ are independent over 2 , we see that

$$
M_{2}=T M_{1} \text {. }
$$

We also point out that, if the ideal a has the basis $\left\{\nu_{1}, \nu_{2}, \nu_{3}\right\}$ and

$$
\phi\left(\begin{array}{l}
\nu_{1} \\
\nu_{2} \\
\nu_{3}
\end{array}\right)=\psi T\left(\begin{array}{l}
\tau_{1} \\
\tau_{2} \\
\tau_{3}
\end{array}\right)
$$

where $\phi \in\left[\tau_{1}, \tau_{2}, \tau_{3}\right]=\mathfrak{b}, \psi \in \mathfrak{a}$, and $T \in G L_{3}(\mathscr{Z})$, then $\left\{\tau_{1}, \tau_{2}, \tau_{3}\right\}$ is a basis of $\mathfrak{b}$. We say that two ideals $\mathfrak{a}$ and $\mathfrak{b}$ are equivalent, written $\mathfrak{a} \sim \mathfrak{b}$, when there exists two nonzero principal ideals $[\alpha],[\beta]$ such that $[\alpha] \mathfrak{a}=[\beta] \mathfrak{b}$. Thus, if $(2.7)$ is true, we see that $\mathfrak{a} \sim \mathfrak{b}$.

If $\left\{\omega_{1}, \omega_{2}, \omega_{3}\right\}$ is any basis of $\mathcal{2}[\delta]$, we know (see, for example, Landau [8, p. 117]) that any ideal $a$ of $2[\delta]$ has a basis $\left\{\alpha_{1}, \alpha_{2}, \alpha_{3}\right\}$, where

$$
\alpha_{1}=a_{11} \omega_{1}, \quad \alpha_{2}=a_{21} \omega_{1}+a_{22} \omega_{2}, \quad \alpha_{3}=a_{31} \omega_{1}+a_{32} \omega_{2}+a_{33} \omega_{3} .
$$


Here all $a_{i j} \in \stackrel{:}{=}$, and $a_{11}, a_{22}, a_{33}>0$. Since $\stackrel{()}{=}[\delta]$ has a basis with $\omega_{1}=1$, we can assume that a has a basis $\left\{\alpha_{1}, \alpha_{2}, \alpha_{3}\right\}$, where $\alpha_{1}>0$ and $\alpha_{1} \in \cong$. We define $L($ a ) to

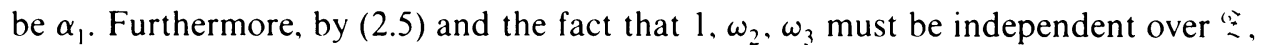
we see that there is only one possible value of such an $\alpha_{1}$ for any ideal $\mathfrak{a}$. In fact it is the least positive rational integer in $a$. If we let $N(a)$ denote the norm of $a$, then $N(a)=a_{11} a_{22} a_{33}$, and we see that $L(a) \mid N(a)$.

If we put $\quad h=\left\langle 1, \alpha_{2} / \alpha_{1}, \alpha_{3} / \alpha_{1}\right\rangle$, we say that $h$ is the l-lattice which corresponds to the ideal $a$. In view of the above remarks there can only be one such 1-lattice. Notice that if $s$ is a l-lattice which corresponds to a and $a=[m] \mathfrak{b}$, where $m \in$ and $\mathfrak{b}$ is an ideal of $\stackrel{(?)}{\delta} \delta$, then ( $h$ also corresponds to $\mathfrak{b}$.

From the above definitions and results, it is clear that if 6 is a 1-lattice which corresponds to an ideal $a$ and $h^{*}$ is a 1-lattice which corresponds to an ideal $b$, then $a \sim b$ if and only if $\mathfrak{h}_{\mathcal{H}} \sim \boldsymbol{H}^{*}$. We can be more specific about this in

Lemma 2.1. Let (hi be a l-lattice which corresponds to the ideal a and let $\mathrm{R} *$ be a

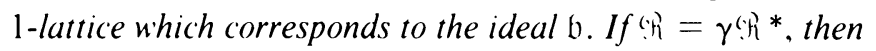

$$
[L(\mathfrak{b})] \mathfrak{a}=[L(\mathfrak{a}) \gamma] \mathfrak{b}
$$

If $\mathfrak{a} \sim \mathfrak{b}$ and $[\phi] \mathfrak{a}=[\psi] \mathfrak{b}$, where $\phi, \psi \in \stackrel{\mathfrak{l}}{\sim}[\delta]$, then

$$
\left(h=\gamma^{*}\right)^{*} \text {, }
$$

where $\gamma=L(\mathfrak{b}) \psi / L(\mathfrak{a}) \phi$.

Proof. Follows easily from (2.3), (2.4), and (2.5).

We also have

Lemma 2.2. Let $h^{2}$ and $h^{*}$ be 1-lattices. If $h$ corresponds to the ideal a and $\mathrm{G}_{\mathrm{h}} \sim \mathrm{G}^{*}$, then there exists an ideal buch that $\mathfrak{h}^{*}$ corresponds to $\mathfrak{b}$.

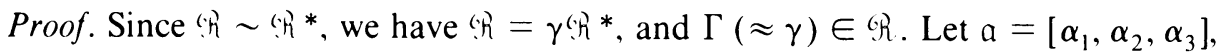
where $\left\{\alpha_{1}, \alpha_{2}, \alpha_{3}\right\}$ is a basis of $a$ and $\alpha_{1}=L(a)$. Then $R=\left\langle 1, \alpha_{2} / \alpha_{1}, \alpha_{3} / \alpha_{1}\right\rangle$, and ( $^{*}=\left\langle\gamma^{-1}, \gamma^{-1} \alpha_{2} / \alpha_{1}, \gamma^{-1} \alpha_{3} / \alpha_{1}\right\rangle$. Now $\ell^{*}=\left\langle 1, \kappa_{1}, \kappa_{2}\right\rangle, \kappa_{1}, \kappa_{2} \in \mathcal{Y}$; thus, if we let

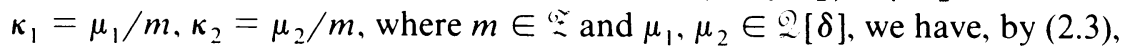

$$
m\left(\begin{array}{l}
\alpha_{1} \\
\alpha_{2} \\
\alpha_{3}
\end{array}\right)=\alpha_{1} \gamma T\left(\begin{array}{l}
m \\
\mu_{1} \\
\mu_{2}
\end{array}\right),
$$

where $T \in G L_{3}\left(\stackrel{(\because)}{=}\right.$. Since $\Gamma \in \mathfrak{R}$, it follows that $\alpha_{1} \gamma \in \mathfrak{a}$; hence, $\mathfrak{b}=\left\{m l_{1}+\mu_{1} l_{2}\right.$ $\left.+\mu_{2} l_{3} \mid l_{1}, l_{2}, l_{3} \in \stackrel{2}{=}\right\}$ is an ideal of $\mathcal{Q}[\delta]$, and $\mathfrak{R}^{*}$ corresponds to $\mathfrak{b}$.

We conclude this section with a consequence of the following important definition.

Definition. Let $\varsigma_{1}$ and $\delta_{2}$ be 1-lattices, and suppose that $\delta_{1}$ corresponds to the ideal $a_{1}$ and $\varsigma_{2}$ corresponds to the ideal $a_{2}$. We define the lattice $\delta_{1} \delta_{2}$ to be the 1-lattice which corresponds to the ideal $a_{1} a_{2}$.

From this definition and Lemmas 2.1 and 2.2, it follows that, if $\varsigma_{i}(i=1,2,3,4)$ are 1-lattices, $\varsigma_{i}$ corresponds to the ideal $a_{i}(i=1,2)$ and $\varsigma_{1} \sim \delta_{3}, \varsigma_{2} \sim \delta_{4}$, then $\varsigma_{1} \varsigma_{2} \sim \delta_{3} \varsigma_{4}$. 
3. Outline of the Method. In this section, we will sketch the overall method which we will use to determine $R$ and $h$ for $2(\delta)$. We first require some further results concerning 1-lattices.

Let $R$ be a lattice as given by $(2.1)$, and let $\Omega(\approx \omega) \in R$. We define the normed body of $\Omega$, written $)(\Omega)$, to be

$$
\Re(\Omega)=\left\{(x, y, z)\left|(x, y, z) \in \mathcal{E}_{3},\right| x|\leqslant| \omega \mid, y^{2}+z^{2} \leqslant \omega^{\prime} \omega^{\prime \prime}\right\},
$$

where $\varepsilon_{3}$ is Euclidean 3 -space. We say that $\Omega(\neq(0,0,0))$ is a relative minimum of $R$ if

$$
\Re(\Omega) \cap \leftrightarrow R=\{(0,0,0), \Omega,-\Omega\} .
$$

If $\Omega(\approx \omega)$ and $\Phi(\approx \phi)$ are relative minima of $R$ such that

$$
0<\phi<\omega, \quad \phi^{\prime} \phi^{\prime \prime}>\omega^{\prime} \omega^{\prime \prime},
$$

and there does not exist a $\Psi(\approx \psi) \in \mathcal{R}$ such that

$$
\phi<\psi<\omega, \quad \psi^{\prime} \psi^{\prime \prime}<\phi^{\prime} \phi^{\prime \prime},
$$

we call $\Omega$ the relative minimum adjacent to $\Phi$. (Actually Voronoi [15] used the term relative minimum of the second kind adjacent to $\Phi$.)

LemMa 3.1 (VORONOI [15, Sections 21, 22]). Let $\mathrm{R}$ be a l-lattice in which the point $(1,0,1)$ is a relative minimum, and let $\Theta(\approx \theta)$ be the relative minimum adjacent to $(1,0,1)$ in $R$. Then there exists $\Psi(\approx \psi) \in \Re$ such that $\Re=\langle 1, \theta, \psi\rangle$.

Proof. Let $R=\left\langle 1, \lambda_{2}, \lambda_{3}\right\rangle, \theta=a+b \lambda_{2}+c \lambda_{3}(a, b, c \in \mathscr{Z}), d=\operatorname{gcd}(b, c)$. If $d>1$, find $r$ such that $r \equiv a(\bmod d)$ and $|r| \leqslant d / 2$. Put $\phi=|(r-\theta) / d|$. Clearly, there exists $\Phi \in \mathcal{R}$ such that $\Phi \approx \phi$. Also, $0<\phi<|r / d|+|\theta / d| \leqslant 1 / 2+\theta / 2<\theta$ and $\left|\phi^{\prime}\right| \leqslant|r / d|+\left|\theta^{\prime} / d\right| \leqslant 1 / 2+\left|\theta^{\prime} / 2\right|<1$. As this contradicts the definition of $\theta$, we must have $d=1$. Since $\operatorname{gcd}(b, c)=1$, there exist $y, z \in \mathscr{Z}$ such that $b z-y c=1$. Putting $\psi=x+y \lambda_{2}+z \lambda_{3}$ for any $x \in \mathscr{Z}$, we see that

$$
\left(\begin{array}{l}
1 \\
\theta \\
\psi
\end{array}\right)=T\left(\begin{array}{c}
1 \\
\lambda_{2} \\
\lambda_{3}
\end{array}\right)
$$

where

$$
T=\left(\begin{array}{lll}
1 & 0 & 0 \\
a & b & c \\
x & y & z
\end{array}\right) \quad \text { and } \quad T \in G L_{3}(\mathscr{Z})
$$

Hence $\{1, \theta, \psi\}$ is a basis of $R$.

We call a 1-lattice in which $(1,0,1)$ is a relative minimum a reduced lattice, and we call a basis of the type $\{1, \theta, \psi\}$ in Lemma 3.1 a reduced basis.

Let $a$ be a primitive ideal (an ideal with no rational integer divisors). We say that $a$ is a reduced ideal if and only if there does not exist $\alpha \in \mathfrak{a}$ such that both $|\alpha|<L(a)$ and $\left|\alpha^{\prime}\right|<L(a)$ hold. (This is essentially the idea of Berwick [2, pp. $418-419$ ] and [3].) It can be easily deduced from the results in [15, Section 39] that

$$
L(\mathfrak{a})<\sqrt{|\Delta| / 3}
$$

when $a$ is reduced. Note that $a$ is reduced if and only if its corresponding lattice is reduced. 
If $R_{1}$ is the lattice which has as a basis an integral basis of $\mathscr{2}[\delta]$, then $R_{1}$ is a reduced lattice, because $(1,0,1)$ must be a relative minimum in such a lattice. Let $R$ be any reduced lattice, and consider the sequence

$$
\Theta_{1}=(1,0,1), \Theta_{2}, \Theta_{3}, \ldots, \Theta_{n}, \ldots,
$$

where $\Theta_{i+1}$ is the relative minimum adjacent to $\Theta_{i}$ for $i=1,2,3, \ldots, n, \ldots$ We call this sequence the chain of relative minima of $R$. We know (see [6] or [18]) that such a chain will be of infinite extent. Also, if $\theta_{n} \approx \Theta_{n}$, then $\theta_{1}<\theta_{2}<\theta_{3}<\cdots<\theta_{n}<\cdots$. Hence, if $\Phi(\approx \phi)$ is a relative minimum of $Q R$ with $\phi>1$, then $\Phi=\Theta_{k}$ for some $k \geqslant 2$.

If $\varepsilon(>1)$ is any unit of $\Re_{1}$, we see that, since $N(\varepsilon)=1$, we must have $E(\approx \varepsilon)$ a relative minimum of $\Re_{1}$, and therefore $E=\Theta_{k}$ in the chain (3.1) in $\Re_{1}$. Thus, if we can find the chain (3.1) in $\mathscr{G}_{1}$, we should be able to find $\varepsilon_{0}$. In [15] Voronoi described how to find the relative minimum adjacent to $(1,0,1)$ in a reduced lattice. A version of this algorithm suitable for computers is described at some length in Williams, Cormack and Seah [18]. This solves the problem of finding the elements of (3.1). For, we find $\theta_{g}^{(1)}=\theta_{2}$ by using Voronoi's algorithm and embed $1, \theta_{g}^{(1)}$ in a reduced basis $\left\{1, \theta_{g}^{(1)}, \theta_{h}^{(1)}\right\}$ of $\Omega_{1}$. Let $\Re_{2}=\left\langle 1,1 / \theta_{g}^{(1)}, \theta_{h}^{(1)} / \theta_{g}^{(1)}\right\rangle$. Clearly $\mathscr{R}_{2}$ is a reduced lattice, and we find $\theta_{g}^{(2)}$, the relative minimum adjacent to 1 in $\Re_{2}$. We have $\theta_{3}=\theta_{g}^{(1)} \theta_{g}^{(2)}$. If $\left\{1, \theta_{g}^{(n)}, \theta_{h}^{(n)}\right\}$ is a reduced basis of $\Re_{n}$, then define $\Re_{n+1}$ by

$$
\stackrel{o R}{n+1}_{n}=\left\langle 1,1 / \theta_{g}^{(n)}, \theta_{h}^{(n)} / \theta_{g}^{(n)}\right\rangle
$$

and continue the process by finding $\theta_{g}^{(n+1)}$, the relative minimum adjacent to $(1,0,1)$ in the reduced lattice ${ }^{2} R_{n+1}$. We have

$$
\theta_{n+2}=\theta_{g}^{(1)} \theta_{g}^{(2)} \cdots \theta_{g}^{(n+1)}
$$

Hence

$$
\varepsilon_{0}=\theta_{k}=\prod_{i=1}^{k-1} \theta_{g}^{(i)}
$$

and

$$
R=\log \varepsilon_{0}=\sum_{i=1}^{k-1} \log \theta_{g}^{(i)}
$$

Also, $\Re_{1}=\theta_{n} \Re_{n}$; hence, $\Re_{n} \sim \Re_{1}$. We further point out that if $\varepsilon_{0}=\theta_{k}$, then $\Re_{k-1+m}=\Re_{m}, \Re_{s(k-1)+m}=\Re_{m}$ and $\theta_{(k-1) s+m}=\varepsilon_{0}^{s} \theta_{m}$.

What we now wish to do is to find, as Shanks did in the case of $2(\sqrt{D})$, a method of getting $\theta_{m}$, where $\theta_{m}$ is close to $\theta_{n}^{2}$, when we know $\theta_{n}$. In order to do this, we first require two results which are due essentially to Voronoi [15, Section 39]. As we will often have need of $\Re(1,0,1)$, we will denote it by $\bigodot_{1}$. We also denote the set of points $\left\{(x, y, z) \mid(x, y, z) \in \mathcal{E}_{3}, y^{2}+z^{2} \leqslant 1\right\}$ by $\circlearrowright$.

THEOREM 3.2. Let $R$ be any 1-lattice. There exists a reduced lattice $\Re *$ such that $\Re^{*} \sim \Re$. Further, there exists $\Gamma(\approx \gamma) \in \Re$ such that $\gamma^{*}=\mathscr{R}$ and $0<\gamma \leqslant 1$, $\left|\gamma^{\prime}\right| \leqslant 1$. 
Proof. Let $h=\left\langle 1, \lambda_{2}, \lambda_{3}\right\rangle$. If $(1,0,1)$ is a relative minimum of $h$, we are finished. If $(1,0,1)$ is not a relative minimum of $R$, then there must be a $P$ $(\approx \rho) \in \mathscr{R}$ such that

$$
\rho \neq 0, \quad|\rho|<1, \quad\left|\rho^{\prime}\right|<1 .
$$

Since any such $\rho$ must be of the form $\rho=a+b \lambda_{2}+c \lambda_{3}$, where $a, b, c \in$ \& , there can only be a finite number of points $P(\approx \rho) \in(h$ which satisfy (3.2). Let this number be $n$.

If $d=\operatorname{gcd}(b, c)$ and $d>1$, let $\theta=(\rho-r) / d$, where $|r| \leqslant d / 2$ and $r \equiv a(\bmod d)$. We have $\theta \neq 0,|\theta| \leqslant|\rho / d|+|r / d| \leqslant 1,\left|\theta^{\prime}\right| \leqslant\left|\rho^{\prime} / d\right|+|r / d|<1$, and $\theta=(a-r) / d$ $+(b / d) \lambda_{1}+(c / d) \lambda_{2}$. Thus we may assume with no loss of generality that we can select a fixed $\Theta(\approx \theta)$ of $i h$ such that

$$
\theta \neq 0, \quad|\theta|<1, \quad\left|\theta^{\prime}\right|<1,
$$

and

$$
\boldsymbol{\theta}=a+b \lambda_{1}+c \lambda_{2} \text { with } \operatorname{gcd}(b, c)=1 .
$$

By using the argument of Lemma 3.1, we see that we can embed $\theta$ in a basis of $h$.

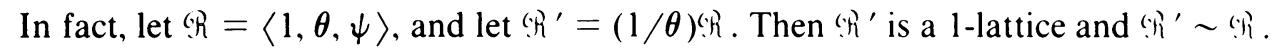
If $\left(R{ }^{\prime}\right.$ is a reduced lattice, $\gamma=|\theta|$ and we are finished; if $(R$ ' is not a reduced lattice. there must exist $\Phi(\approx \phi) \in R^{\prime}$ such that

$$
\phi \neq 0, \quad|\phi|<1, \quad\left|\phi^{\prime}\right|<1 .
$$

Again, there can only be a finite number of such elements in $\left(R^{\prime}\right.$, and we denote this number by $m$. If $\Phi$ is any such element, then

$$
\phi=l_{1} / \theta+l_{2}+l_{3} \psi / \theta, \text { where } l_{1}, l_{2}, l_{3} \in
$$

hence, $X\left(\approx \chi=\theta \phi=l_{1}+l_{2} \theta+l_{3} \psi\right)$ is an element of $(h$ and $\chi \neq 0,|\chi|=|\theta \phi|<1$. $\left|\chi^{\prime}\right|=\left|\theta^{\prime} \phi^{\prime}\right|<1$ by (3.3) and (3.4). It follows that $m \leqslant n$. Since, however, $\phi \theta \neq \theta$, we see that $m \neq n$; thus, $m<n$. We can then repeat the above argument on ${ }^{\prime} h$ ' instead of $\left(R\right.$. Since, in each new 1-lattice ${ }^{(j)}\left(\sim R^{(j-1)}\right)$ that we develop, we have fewer and fewer elements $P(\approx \rho)$ such that (3.2) holds, we must eventually reach the point where we have a $R^{(k)}$ such that $R_{R^{(k)}}$ is reduced. The result of the theorem follows on putting $\mathscr{R}^{*}=G_{R}^{(k)}$ and noting that $Q_{R}^{(k)} \sim \mathscr{R}$.

COROLlaRY. If a is any primitive ideal of $\mathcal{2}[\delta]$, there exists $\lambda \in$ a and a reduced ideal $\mathfrak{b}$ of $\mathcal{Q}[\delta]$ such that

$$
[\lambda] \mathfrak{b}=[L(\mathfrak{b})] \mathfrak{a}
$$

and

$$
L(\mathfrak{a})^{-1} \leqslant \lambda \leqslant L(\mathfrak{a}), \quad 1 \leqslant\left|\lambda^{\prime}\right| \leqslant L(\mathfrak{a}) .
$$

Proof. By the theorem there exists $\Gamma(\approx \gamma) \in \mathscr{R}$ and a reduced lattice $R^{*}$ such that $\gamma^{\mathscr{R}} *=\mathscr{R}, 0<\gamma \leqslant 1,\left|\gamma^{\prime}\right| \leqslant 1$. By Lemma 2.2 there exists an ideal $\mathfrak{b}$ of $\mathcal{Q}[\delta]$ such that $\Re *$ corresponds to $\mathfrak{b}$. By Lemma 2.1

$$
[L(\mathfrak{b})] \mathfrak{a}=[L(\mathfrak{a}) \gamma] \mathfrak{b} .
$$


Put $\lambda=L(\mathfrak{a}) \gamma \in \mathfrak{a}$. We must have $0<\lambda \leqslant L(\mathfrak{a}),\left|\lambda^{\prime}\right| \leqslant L(\mathfrak{a})$. Since $\lambda \in \mathfrak{a}$, we have $\lambda \equiv 0(\bmod a)$ and $N(a) \mid N(\lambda)$. Thus $N(\lambda)=\lambda\left|\lambda^{\prime}\right|^{2} \geqslant N(a)$, and

$$
\lambda=N(\lambda) /\left|\lambda^{\prime}\right|^{2} \geqslant N(a) / L(a)^{2} \geqslant L(a)^{-1} .
$$

THEOREM 3.3. Let $\%$ and $\varphi^{*}$ be two reduced lattices; then $\% \sim R *$ if and only if $\Leftrightarrow=\theta^{*}$, where $\Theta(\approx \theta)$ is a relative minimum of $h$.

Proof. Let ( h have $\{1, \mu, \nu\}$ as a reduced basis, and let $h^{*}$ have $\{1, \phi, \psi\}$ as a

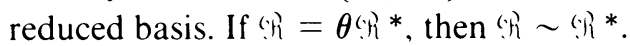

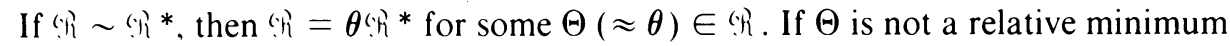
of $(h$, then there must exist $\Omega(\approx \omega) \in(R$ such that $\Omega \in \Re(\Theta)$, and $\Omega \neq(0,0,0)$,

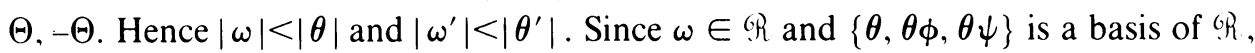
we must have $a, b, c \in$ such that $\omega=a \theta+b \theta \phi+c \phi \psi$. If $\rho=\omega / \theta=a+b \phi+$ c $\psi$ and $P \approx \rho$, then $P \in$ : $^{*}$. Since $|\rho|<|\omega / \theta|<1$ and $\left|\rho^{\prime}\right|=\left|\omega^{\prime}\right|<1$, we see that $P \in \mathcal{E}_{1}$. Since $P \neq(0,0,0)$, this is impossible; thus, $\Theta$ is a relative minimum of $\Omega$.

Let $a$ be any nonzero ideal, and let $(R$ be the lattice which corresponds to $a$. By Theorem 3.3 we see that Voronoi's algorithm can be used to find all the reduced ideals which are in the same ideal class as $a$. In fact, if we put $\mathscr{C}_{R}=\mathscr{R}_{1}, a_{1}=a=0$, then with Voronoi's algorithm we can find $a_{n}, \Re_{n}, \theta_{n} \in \mathcal{Z}[\delta]$ such that $\mathscr{G}_{n}$ is the lattice which corresponds to $a_{n}$ and

$$
\left[\theta_{n}\right] a_{n}=\left[L\left(a_{n}\right)\right] \text {. }
$$

Since $h$, above corresponds to the ideal $\mathfrak{o}=2[\delta]$, we must have $\mathfrak{o}^{2}=\mathfrak{o}$ and $\leftrightarrow_{1}^{2}=R_{1}$. Also, since $R_{1} \sim R_{1}$ and $R_{k} \sim R_{1}$, we have

$$
R_{j} \cdot R_{k} \sim R_{1}^{2} \sim R_{1}
$$

by the remark at the end of Section 2. By Theorem 3.2, there exists a reduced lattice $S$ such that $S \sim \Re_{j} \Re_{k} \sim \Re_{1}$. Let $\Gamma(\approx \gamma) \in \Re_{j} \Re_{k}$ such that $\gamma \delta=\Re_{j} \Re_{k}$. Then by Lemma 2.2 we get

$$
\Re_{1}=t \theta_{j} \theta_{k} \Re_{j}{ }^{Q} R_{k},
$$

where $t=L\left(a_{j} a_{k}\right) / L\left(a_{j}\right) L\left(a_{k}\right)$; thus,

$$
R_{1}=\phi \varsigma,
$$

where

$$
\phi=\gamma t \theta_{j} \theta_{k}
$$

Also, since $\left|\gamma^{\prime}\right| \leqslant 1,0<\gamma \leqslant 1, L\left(a_{j}\right) L\left(a_{k}\right) \geqslant L\left(a_{j} a_{k}\right)>0,\left|\theta^{\prime}{ }_{j}\right|<1,\left|\theta^{\prime}{ }_{k}\right|<1$, we get $\left|\phi^{\prime}\right|<1$. Thus $\phi>1$ and $\phi \leqslant \theta_{j} \theta_{k}$.

Since $\varsigma$ and $\Omega_{1}$ are both reduced lattices, we see by Theorem 3.3 that $\Phi(\approx \phi)$ is a relative minimum of $\Re_{1}$. Hence $\phi=\theta_{m}$ for some $m$ and $\delta=\Re_{m}$. If $a_{j} a_{k}=[s] a$, where $a$ is a primitive ideal, we see that $s L(a)=L\left(a_{j} a_{k}\right)$. Also, since $L(a)^{-1} \leqslant$ $\gamma L(a) \leqslant L(a)$ and $L\left(a_{i}\right), L\left(a_{j}\right)<\sqrt{|\Delta| / 3}$, we have $t \gamma=s L(a) \gamma / L\left(a_{j}\right) L\left(a_{k}\right)>$ $(3 /|\Delta|)^{2}$. Thus, if $j$ and $k$ are such that $\theta_{j}$ and $\theta_{k}$ are large, then we have $\theta_{m} \simeq \theta_{j} \theta_{k}$ 
and $m \simeq j+k$. In fact, when $\delta^{3}=D \in \stackrel{\sim}{*}$, and $j=k=n$, we will see in Section 6 that

$$
\theta_{m}=\theta_{n}^{2} \gamma / w
$$

where $w \in$ and $w \mid 3 g_{1} g_{2}$.

In the sections that follow, we will show how to determine $R_{n}^{2}, \gamma$ and $S$, given $R_{n}$. We call our algorithm for finding $\gamma$ and $s$ from $R_{n}$ the doubling procedure, as it allows us to find

$$
\log \phi=2 \log \theta_{n}+\log \gamma-\log w,
$$

when we have $Q_{R}$ and $\log \theta_{n}$. The difficulty in using the doubling idea is that, while we can now skip over many of the steps of Voronoi's algorithm, we need to know where we are going in order not to skip an important step such as the $k$ th step when $\theta_{k}=\varepsilon_{0}$. Thus, we need to have some estimate of $R$, which is what we are trying to find. We can, however, estimate $h R$ by using the Euler product method. For the pure cubic case (see, for example, Barrucand, Williams and Baniuk [1]) we have

$$
h R=J \sqrt{3} \Phi(1) / 2 \pi
$$

where $\Phi(1)=\zeta_{\mathfrak{K}}(1) / \zeta(1)$ is given by the Euler product

$$
\Phi(1)=\prod_{q} f(q) .
$$

Here, the product is taken over all the rational primes $q$ and $f(q)$ is given by the following:

(i) if $q \mid J$, then $f(q)=1$;

(ii) if $3 \nmid J$, then $f(3)=3 / 2$;

(iii) if $q \equiv-1(\bmod 3)$ and $q \nmid J$, then $f(q)=q^{2} /\left(q^{2}-1\right)$;

(iv) if $q \equiv 1(\bmod 3)$ and $q \nmid J$, then

$$
f(q)= \begin{cases}q^{2} /(q-1)^{2} & \text { when }(D / q)_{3}=1 \\ q^{2} / q^{2}+q+1 & \text { when }(D / q)_{3} \neq 1\end{cases}
$$

Since

$$
c_{1}=\prod_{q \equiv-1(\bmod 3)} q^{2} /\left(q^{2}-1\right)
$$

converges (approximate value 1.414064387), we can approximate $\Phi(1)$ by evaluating the product over the primes $q \equiv 1(\bmod 3)$ only. The real difficulty lies in knowing how many primes to use. Put $Q>3$,

$$
F(Q)=\prod_{\substack{q \leqslant Q \\ q \equiv 1(\bmod 3)}} f(q),
$$

and

$$
c_{2}=f(3) c_{1} \prod_{\substack{q J \\ q \equiv-1(\bmod 3)}}\left(q^{2}-1\right) / q^{2} \leqslant 3 / 2 c_{1} \text {. }
$$


We used $Q=10^{6}$ for $D \lesssim 2 \times 10^{7}, Q=10^{7}$ for $D \simeq 2 \times 10^{8}$, and $Q=10^{8}$ for $D>10^{9}$ and evaluated our estimate $E$ of $h R$ from

$$
E=\sqrt{3} c_{2} J F(Q) / 2 \pi
$$

We discuss in Section 10 how good an approximation we expect $E$ to be of $h R$.

If we divide $E$ by $2^{\kappa}$, we get

$$
E=2^{\kappa} U \text {. }
$$

Voronoi's algorithm can be used to find $\Re_{n}$ and

$$
R_{n}=\log \theta_{n}=\sum_{i=1}^{n-1} \log \theta_{g}^{(i)},
$$

where $R_{n}<U$ and $R_{n+1}>U$. The doubling process can then be used $\kappa$ times to find $Q_{R}$, and

$$
R_{t} \simeq 2^{\kappa} R_{n} \text {. }
$$

We can then resume using Voronoi's algorithm, starting at $\Re_{t}$, to find $\Re_{t+1}$, $Q_{R} R_{t+2}, \ldots$ until we find $R_{k}$ such that $N\left(\theta_{k}\right)=1$. Then probably $R_{k}=\log \theta_{k}=h R$. What we do not know is $h$ or $R$. The next step, therefore, is to find $h$. Since $R=\log \varepsilon_{0}>R_{n}$ and $R \leqslant R_{k}$, we have $h<R_{k} / R_{n} \simeq 2^{\kappa}$. We now attempt to find all the primes less than $R_{k} / R_{n}$ which divide $h$. If $p$ is such a prime, then $R_{s}=h R / p$ for some $s$. If we let

$$
h R / p=2^{\kappa} U_{p},
$$

we can repeat the procedure described above to find $R_{u}$ and $R_{u}$ such that $R_{u}<h R / p$ and $h R / p$ is close in value to $R_{u}$. We can then apply Voronoi's algorithm to $\Re_{u}$ to find $\Re_{u+1}, \Re_{u+2}, \ldots$ until we either find $\Re_{v}$ such that $N\left(\theta_{v}\right)=1$, in which case $p \mid h$, or we find $R_{v}$ such that $R_{v}>h R / p$, in which case $p \nmid h$. If we find a $p$ which does divide $h$, we must replace $\Re_{k}$ by $R_{v}, R_{k}$ by $R_{v}$, and repeat this procedure to determine the precise power of $p$ that divides $h$. When this process has been completed for all primes less than $R_{k} / R_{n}$, we have the value of $h$ and also that of $R=R h / h$.

This has been only a brief account of the algorithm for finding $h$ and $R$ from an estimate $E$ of $h R$. We give a much more detailed algorithm in Section 10.

4. Some Simple Properties of 1-Lattices Equivalent to $\Omega_{1}$. Let $R$ be any 1-lattice such that $R \sim \Re_{1}$, and let $\Re_{1}$ have basis $\{1, \bar{\mu}, \bar{\nu}\}$, where

$$
\bar{\mu}=\left(\bar{m}_{1}+\bar{m}_{2} \delta+\bar{m}_{3} \delta^{2}\right) / \sigma, \quad \bar{\nu}=\left(\bar{n}_{1}+\bar{n}_{2} \delta+\bar{n}_{3} \delta^{2}\right) / \sigma, \quad \sigma>0,
$$

$\sigma, \bar{m}_{i}, \bar{n}_{i} \in \mathscr{Z}(i=1,2,3)$ and $\operatorname{gcd}\left(\bar{m}_{1}, \bar{m}_{2}, \bar{m}_{3}, \bar{n}_{1}, \bar{n}_{2}, \bar{n}_{3}, \sigma\right)=1$. When $\mathcal{K}$ is a pure cubic field, values for the integers $m_{i}, n_{i}$, and $\sigma$ here are given in Section 1. Put $e=\bar{m}_{2} \bar{n}_{3}-\bar{n}_{2} \bar{m}_{3}$. Since $\Re \sim \Re_{1}$, there must exist $\psi \in \mathcal{L}[\delta]$ such that $\Re_{1}=\psi \Re$ and

$$
\left(\begin{array}{l}
1 \\
\mu \\
\nu
\end{array}\right)=\frac{1}{\psi} T\left(\begin{array}{c}
1 \\
\bar{\mu} \\
\bar{\nu}
\end{array}\right)
$$


where $\stackrel{R}{R}=\langle 1, \mu, \nu\rangle$ and $T \in G L_{3}(\stackrel{s}{=})$. Let

$$
\mu=\left(m_{1}+m_{2} \delta+m_{3} \delta^{2}\right) / \rho, \quad \nu=\left(n_{1}+n_{2} \delta+n_{3} \delta^{2}\right) / \rho,
$$

where $\rho>0, \rho, m_{i}, n_{i} \in(i=1,2,3)$ and $\operatorname{gcd}\left(m_{1}, m_{2}, m_{3}, n_{1}, n_{2}, n_{3}, \rho\right)=1$. Put $f=m_{2} n_{3}-n_{2} m_{3}$.

We point out that if

$$
M=\left(\begin{array}{ccc}
\rho & 0 & 0 \\
m_{1} & m_{2} & m_{3} \\
n_{1} & n_{2} & n_{3}
\end{array}\right),
$$

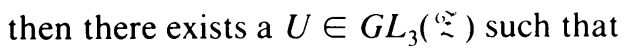

$$
U M=\left(\begin{array}{ccc}
\rho & 0 & 0 \\
m & b & 0 \\
n & n^{\prime} & n^{\prime \prime}
\end{array}\right),
$$

where $n^{\prime \prime}=\operatorname{gcd}\left(n_{3}, m_{3}\right)$ and $b=f / n^{\prime \prime} \in$. We call the corresponding basis

$$
\left\{1, \frac{m+b \delta}{\rho}, \frac{n+n^{\prime} \delta+n^{\prime \prime} \delta^{2}}{\rho}\right\}
$$

a canonical basis of $\left(R\right.$. Note that $\operatorname{gcd}\left(m, b, n, n^{\prime}, n^{\prime \prime}, \rho\right)=1$.

We can find $U$ by the following procedure. We may assume that $m_{3}>0$; for, if this is not so, we can replace $\mu$ by $-\mu$. If we use the Euclidean algorithm to find

$$
\begin{gathered}
n_{3}=q_{0} m_{3}+r_{0} \quad\left(0<r_{0}<m_{3}\right), \\
m_{3}=q_{1} r_{0}+r_{1} \quad\left(0<r_{1}<r_{0}\right), \\
\cdots \cdots q_{k} \ldots \ldots+r_{k} \quad\left(r_{k}=0\right), \\
r_{k}=q_{k} r_{k}+r_{k}
\end{gathered}
$$

then we see that $r_{h}=n^{\prime \prime}$ and $Q_{k} Q_{k}, \cdots Q_{0}$, where

$$
Q_{i}=\left(\begin{array}{ccc}
1 & 0 & 0 \\
0 & -q_{1} & 1 \\
0 & 1 & 0
\end{array}\right)
$$

is a suitable matrix for $U$.

We now prove a more general version of Theorem 3.1 of [18].

THEOREM 4.1. In the notation above, if $N=N(\psi)$, then $|N|=\rho^{2}|e| / \sigma^{2}|f|$ : also, $f \mid \rho$.

Proof. From (4.1) we get

$$
\begin{aligned}
\sigma \psi & =x_{11}+x_{12} \delta+x_{13} \delta^{2}, \\
\sigma \psi \mu & =x_{21}+x_{22} \delta+x_{23} \delta^{2}, \\
\sigma \psi \nu & =x_{31}+x_{32} \delta+x_{33} \delta^{2},
\end{aligned}
$$

where $x_{i j} \in \mathscr{Z}(i=1,2,3 ; j=1,2,3)$. If

$$
\bar{M}=\left(\begin{array}{ccc}
\sigma & 0 & 0 \\
\bar{m}_{1} & \bar{m}_{2} & \bar{m}_{3} \\
\bar{n}_{1} & \bar{n}_{2} & \bar{n}_{3}
\end{array}\right),
$$


then $X=\left(x_{i j}\right)_{3 \times 3}=T \bar{M}$ and $|X|= \pm e \sigma$. Since $\psi \in \stackrel{\mathcal{O}}{\sim}[\delta]$, we have $\lambda=\psi^{\prime} \psi^{\prime \prime} \in \mathcal{Q}[\delta]$, and therefore $\sigma \lambda=l_{1}+l_{2} \delta+l_{3} \delta^{2}$, where $l_{i} \in \stackrel{\Xi}{\approx}(i=1,2,3)$. Thus,

$$
\begin{aligned}
\sigma^{2} \lambda \psi & =\sigma^{2} N, \\
\sigma^{2} \lambda \psi \mu & =\sigma^{2} N \mu=u_{1}+u_{2} \delta+u_{3} \delta^{2}, \\
\sigma^{2} \lambda \psi \nu & =\sigma^{2} N \nu=v_{1}+v_{2} \delta+v_{3} \delta^{2},
\end{aligned}
$$

where $u_{i}, v_{i} \in \stackrel{(}{=}(i=1,2,3)$. Also, since $\lambda \psi \mu$ and $\lambda \psi \nu \in \stackrel{\vartheta}{\sim}[\delta]$, we must have $\operatorname{gcd}\left(u_{1}, u_{2}, u_{3}, v_{1}, v_{2}, v_{3}\right) \equiv 0 \quad(\bmod \sigma)$.

Now

$$
\begin{aligned}
\left(\begin{array}{ccc}
\sigma^{2} N & 0 & 0 \\
u_{1} & u_{2} & u_{3} \\
v_{1} & v_{2} & v_{3}
\end{array}\right) \\
\quad=X\left(\begin{array}{ccc}
l_{1} & l_{2} & l_{3} \\
D l_{3} & l_{1}-C l_{3} & l_{2}+B l_{3} \\
D l_{2}+B D l_{3} & (D-B C) l_{3}-l_{2} C & l_{1}+B l_{2}+\left(B^{2}-C\right) l_{3}
\end{array}\right)
\end{aligned}
$$

By taking determinants of both sides of this expression, we get

$$
\sigma^{2}\left(u_{2} v_{3}-v_{2} u_{3}\right) N= \pm e \sigma N(\sigma \lambda)
$$

by (1.3). Hence

$$
u_{2} v_{3}-v_{2} u_{3}= \pm e \sigma^{2} N
$$

If we put $d^{*}=\operatorname{gcd}\left(u_{1}, u_{2}, u_{3}, v_{1}, v_{2}, v_{3}, \sigma^{2} N\right)$, then $\rho=\sigma^{2}|N| / d^{*}$ and $|f|$ $=|e| \sigma^{2}|N| /\left(d^{*}\right)^{2}$ from (4.4). It follows that $|f|=|e| \rho / d^{*}$ and $|N|=$ $\rho^{2}|e| /|f| \sigma^{2}$. Since $\sigma\left|d^{*}, d^{*}=\rho\right| e|/| f \mid$ and $e \mid \sigma$, we must also have $f \mid \rho$.

THEOREM 4.2. If $\Omega(\approx \omega) \in \mathcal{R}$ and $\omega=\left(q_{1}+q_{2} \delta+q_{3} \delta^{2}\right) / \rho$, then $\sigma|f| \rho$ is a divisor of $N(\rho \omega)$ and $f \mid q_{i}^{\prime}(i=1,2,3)$, where $\rho^{2} \omega^{\prime} \omega^{\prime \prime}=q_{1}^{\prime}+q_{2}^{\prime} \delta+q_{3}^{\prime} \delta^{2}$.

Proof. Since $\Omega \in R$, we have $a, b, c \in \mathscr{Z}$ such that $\omega=a+b \mu+c \nu$. Also, since $\psi, \psi \mu, \psi \nu \in \mathcal{Q}[\delta]$, so is $\lambda=\psi \omega \in \mathcal{Q}[\delta]$. It follows from Theorem 4.1 that

$$
|N(\rho \omega)|=\left|\rho^{3} N(\omega)\right|=\rho^{3}|N(\lambda) / N|=\rho|f| \sigma^{2}|N(\lambda)| /|e| .
$$

Since $e \mid \sigma$ and $N(\lambda) \in \mathscr{Z}$, we have the first part of the theorem. Since

$$
\omega^{\prime} \omega^{\prime \prime} / N(\omega)=1 / \omega=\psi / \lambda=\lambda^{\prime} \lambda^{\prime \prime} \psi / N(\lambda),
$$

we have $\omega^{\prime} \omega^{\prime \prime}=\lambda^{\prime} \lambda^{\prime \prime} \psi / N$. Thus

$$
\left|\rho^{2} \omega^{\prime} \omega^{\prime \prime}\right|=\left|q_{1}^{\prime}+q_{2}^{\prime} \delta+q_{3}^{\prime} \delta^{2}\right|=\sigma^{2}|f|\left|\lambda^{\prime} \lambda^{\prime \prime} \psi\right| /|e| .
$$

Since $e \mid \sigma$ and $\lambda^{\prime} \lambda^{\prime \prime} \psi \in \mathcal{2}[\delta]$, we must have $f \mid q_{i}^{\prime}(i=1,2,3)$.

Now consider the sequence $\left\{\gamma_{i}\right\}(i=1,2,3, \ldots, s)$ where $\gamma_{1}=1$ and $\Gamma_{i}\left(\approx \gamma_{i}\right) \in \Re$ $(i=1,2,3, \ldots, s)$. Put $\mathcal{S}_{1}=\Re, \gamma_{g}^{(i-1)}=\gamma_{i} / \gamma_{i-1}(i=2,3,4, \ldots, s)$, and suppose that 1 and $\gamma_{g}^{(i-1)}$ can be embedded in a basis $\left\{1, \gamma_{g}^{(i-1)}, \gamma_{h}^{(i-1)}\right\}$ of $\delta_{i-1}$. If we define

$$
\delta_{i}=\left(1 / \gamma_{g}^{(i-1)}\right) \delta_{i-1}
$$


then $\mathcal{S}_{i}$ is a 1-lattice and $\mathcal{S}_{i} \sim \mathcal{S}_{i-1}$. Further, if $\left\{1, \mu_{i}, \nu_{i}\right\}$ is any basis of $\mathcal{S}_{i}$, then

$$
\left(\begin{array}{c}
1 \\
\mu_{i} \\
\nu_{i}
\end{array}\right)=\frac{1}{\gamma_{g}^{(i-1)}} T_{i-1}\left(\begin{array}{c}
1 \\
\mu_{i-1} \\
\nu_{i-1}
\end{array}\right)
$$

where $T_{i-1} \in G L_{3}(\mathscr{Z})$ and $\left\{1, \mu_{i-1}, \nu_{i-1}\right\}$ is any basis of $\Sigma_{i-1}$. Hence

$$
\left(\begin{array}{c}
1 \\
\mu_{i} \\
\nu_{i}
\end{array}\right)=\frac{1}{\gamma_{i}} J_{1}\left(\begin{array}{c}
1 \\
\mu \\
\nu
\end{array}\right)=\frac{1}{\gamma_{i} \psi} J_{2}\left(\begin{array}{c}
1 \\
\bar{\mu} \\
\bar{\nu}
\end{array}\right)
$$

where $J_{1}, J_{2} \in G L_{3}(\mathscr{Z})$.

For $\mu_{i}=\left(m_{1}+m_{2} \delta+m_{3} \delta^{2}\right) / \rho_{i}, \nu_{i}=\left(n_{1}+n_{2} \delta+n_{3} \delta^{2}\right) / \rho_{i}$, such that $m_{j}, n_{j}, \rho_{i}$ $\in \mathscr{Z}(j=1,2,3), \rho_{i}>0, \operatorname{gcd}\left(m_{1}, m_{2}, m_{3}, n_{1}, n_{2}, n_{3}, \rho_{i}\right)=1$, put $f_{i}=m_{2} n_{3}-$ $m_{3} n_{2}$. By Theorem 4.1 and (4.5), we see that $f_{i} \mid \rho_{i}$ and

$$
\left|N\left(\gamma_{i} \psi\right)\right|=\rho_{i}^{2}|e| / \sigma^{2}\left|f_{i}\right| \text {. }
$$

We now describe how to find a basis $\left\{1, \mu_{r}, \nu_{r}\right\}$ of $\S_{r}$ when we know the basis $\left\{1, \gamma_{g}^{(r-1)}, \gamma_{h}^{(r-1)}\right\}$ of $\mathcal{S}_{r-1}$.

Let

$$
\gamma_{g}^{(r-1)}=\left(m_{1}+m_{2} \delta+m_{3} \delta^{2}\right) / \rho_{r-1}, \quad \gamma_{h}^{(r-1)}=\left(n_{1}+n_{2} \delta+n_{3} \delta^{2}\right) / \rho_{r-1},
$$

where $\rho_{r-1}, m_{1}, m_{2}, m_{3}, n_{1}, n_{2}, n_{3} \in \stackrel{\mathcal{Z}}{ }, \rho_{r-1}>0$ and

$$
\operatorname{gcd}\left(m_{1}, m_{2}, m_{3}, n_{1}, n_{2}, n_{3}, \rho_{r-1}\right)=1 \text {. }
$$

Define $m_{1}^{\prime}, m_{2}^{\prime}, m_{3}^{\prime}$ by

$$
\rho_{r-1}^{2} \gamma_{g}^{(r-1)^{\prime}} \gamma_{g}^{(r-1)^{\prime \prime}}=m_{1}^{\prime}+m_{2}^{\prime} \delta+m_{3}^{\prime} \delta^{2}
$$

where $m_{1}^{\prime}, m_{2}^{\prime}, m_{3}^{\prime} \in \mathscr{Z}$. Put

$$
d_{1}=\operatorname{gcd}\left(m_{1}^{\prime}, m_{2}^{\prime}, m_{3}^{\prime}\right), \quad \bar{m}_{i}=m_{i}^{\prime} / d_{1}, \quad \bar{\rho}_{r}=\rho_{r-1}^{3} N\left(\gamma_{g}^{(r-1)}\right) / d_{1} .
$$

If $\bar{\rho}_{r}<0$, replace $m_{1}, m_{2}, m_{3}$ by $-m_{1},-m_{2},-m_{3}$ and $\bar{\rho}_{r}$ by $-\bar{\rho}_{r}$. This transformation does not change the values of $m_{1}^{\prime}, m_{2}^{\prime}, m_{3}^{\prime}$.

Define $\bar{n}_{1}, \bar{n}_{2}, \bar{n}_{3}$ by

$$
\bar{n}_{1}+\bar{n}_{2} \delta+\bar{n}_{3} \delta^{2}=\left(\bar{m}_{1}+\bar{m}_{2} \delta+\bar{m}_{3} \delta^{2}\right)\left(n_{1}+n_{2} \delta+n_{3} \delta^{2}\right)
$$

and put

$$
d_{2}=\operatorname{gcd}\left(\bar{n}_{1}, \bar{n}_{2}, \bar{n}_{3}\right), \quad n_{i}^{*}=\bar{n}_{i} / d_{2} \quad(i=1,2,3) .
$$

We have

$$
\begin{aligned}
1 / \gamma_{g}^{(r-1)} & =\rho_{r-1}\left(\bar{m}_{1}+\bar{m}_{2} \delta+\bar{m}_{3} \delta^{2}\right) / \bar{\rho}_{r} \\
\gamma_{h}^{(r-1)} / \gamma_{g}^{(r-1)} & =\left(\bar{n}_{1}+\bar{n}_{2} \delta+\bar{n}_{3} \delta^{2}\right) / \bar{\rho}_{r} .
\end{aligned}
$$

Since

$$
d_{1} d_{2}\left(n_{1}^{*}+n_{2}^{*} \delta+n_{3}^{*} \delta^{2}\right)=\bar{\rho}_{r} \frac{\gamma_{h}^{(r-1)}}{\gamma_{g}^{(r-1)}}=\bar{\rho}_{r} \frac{n_{1}+n_{2} \delta+n_{3} \delta^{2}}{m_{1}+m_{2} \delta+m_{3} \delta^{2}},
$$

it follows that $d_{1} d_{2} \mid \bar{\rho}_{r} n_{i}(i=1,2,3)$. Thus $d_{1} d_{2}$ is a divisor of the $\operatorname{gcd}\left(\bar{\rho}_{r} n_{2}, \bar{\rho}_{r} n_{3}\right)$ and $d_{1} d_{2} \mid \bar{\rho}_{r} f_{r-1}$. By Theorem (4.2), we have $f_{r-1} \rho_{r-1} \mid N\left(\rho_{r-1} \theta_{g}^{(r-1)}\right)$; thus, $f_{r-1} \rho_{r-1} \mid$ $d_{1} \bar{\rho}_{r}$. If $d=\operatorname{gcd}\left(d_{2}, \rho_{r-1}\right)$, then $d f_{r-1} \mid d_{1} \bar{\rho}_{r}$ and $d d_{1} \mid \bar{\rho}_{r} f_{r-1}$; therefore, $d \mid \bar{\rho}_{r}$. We have now proved the following theorem. 
THEOREM 4.3. If $\left\{1, \gamma_{g}^{(r-1)}, \gamma_{h}^{(r-1)}\right\}$ is a basis of $\varsigma_{r-1}$, then $\left\{1, \mu_{r}, \nu_{r}\right\}$ is a basis of $\S_{r}$, where

$$
\begin{aligned}
& \mu_{r}=1 /\left|\gamma_{g}^{(r-1)}\right|=\left(m_{1}^{*}+m_{2}^{*} \delta+m_{3}^{*} \delta^{2}\right) / \rho_{r}, \\
& \nu_{r}=\gamma_{h}^{(r-1)} /\left|\gamma_{g}^{(r-1)}\right|=\left(n_{1}^{*}+n_{2}^{*} \delta+n_{3}^{*} \delta^{2}\right) / \rho_{r},
\end{aligned}
$$

$\rho_{r}=\bar{\rho}_{r} / d>0, m_{i}^{*}=\rho_{r-1} m_{i}^{\prime} / d d_{1}$ and $\operatorname{gcd}\left(m_{1}^{*}, m_{2}^{*}, m_{3}^{*}, n_{1}^{*}, n_{2}^{*}, n_{3}^{*}, \rho_{r}\right)=1$.

By (4.6), and the fact that $\gamma_{g}^{(r-1)}=\gamma_{r} / \gamma_{r-1}$, we find that $d_{1}=N\left(\gamma_{g}^{(r-1)}\right) \rho_{r-1}^{3} / \bar{\rho}_{r}=1$ $f_{r-1}\left|\rho_{r} \rho_{r-1} /\right| f_{r} \mid d$, and therefore

$$
m_{1}^{\prime}=\left|f_{r-1}\right| \rho_{r} m_{i}^{*} /\left|f_{r}\right|
$$

We call the process of finding a basis of $s_{r}$ from a basis of $s_{r-1}$ the invert process. When $\delta^{3}=D$, we have $m_{1}^{\prime}=m_{1}^{2}-D m_{2} m_{3}, m_{2}^{\prime}=D m_{3}^{2}-m_{2} m_{1}, m_{3}^{\prime}=m_{2}^{2}-$ $m_{1} m_{3}, \quad \bar{\rho}_{r}=m_{1} \bar{m}_{1}+D\left(m_{2} \bar{m}_{3}+m_{3} \bar{m}_{2}\right), \quad \bar{n}_{1}=\bar{m}_{1} n_{1}+D\left(\bar{m}_{2} n_{3}+\bar{m}_{3} n_{2}\right), \quad \bar{n}_{2}=$ $\bar{m}_{2} n_{1}+\bar{m}_{1} n_{2}+D \bar{m}_{3} n_{3}, \bar{n}_{3}=\bar{m}_{3} n_{1}+\bar{m}_{2} n_{2}+\bar{m}_{1} n_{3}$. In this case, if $\left|\gamma_{r}^{(r-1)}\right|<1$ and $\left|\gamma_{g}^{(r-1)^{\prime}}\right|<1$, then by Lemma 7 of [15], we have $\delta^{i-1}\left|m_{i}\right|<\rho_{r-1}(i=1,2,3)$, and therefore $\delta^{i-1}\left|m_{i}\right|<2 \rho_{r-1}^{2} /\left|f_{r-1}\right|(i=1,2,3)$. From (4.10), we get $\delta^{i-1}\left|m_{i}^{*}\right|<$ $2 \rho_{r-1}^{2} /\left|f_{r-1}\right|(i=1,2,3)$.

We conclude this section by defining, as was done in [18], what we mean when we say that we transform a basis $\{1, \mu, \nu\}$ of $\mathscr{R}$ by $K$, where $K \in G L_{2}(\mathcal{Z})$. When we replace the basis $\{1, \mu, \nu\}$ by the basis $\{1, \bar{\mu}, \bar{\nu}\}$, where

$$
\begin{gathered}
\left(\begin{array}{c}
1 \\
\bar{\mu} \\
\bar{\nu}
\end{array}\right)=T\left(\begin{array}{l}
1 \\
\mu \\
\nu
\end{array}\right), \\
T=\left(\begin{array}{l|ll}
1 & 0 & 0 \\
\hline t_{21} & K^{T} \\
t_{31}
\end{array}\right),
\end{gathered}
$$

$K^{T}$ is the transpose of $K$ and $t_{21}, t_{31}$ are integers selected such that the new values $\bar{m}_{1}, \bar{n}_{1}$ of $m_{1}$ and $n_{1}$ satisfy $0 \leqslant \bar{m}_{1}, \bar{n}_{1}<\rho$, we say that we have transformed the basis $\{1, \mu, \nu\}$ by $K$. Note that since $|T|= \pm 1$, the new basis is in fact a basis of $R$. Also, if $\bar{\mu}=\left(\bar{m}_{1}+\bar{m}_{2} \delta+\bar{m}_{3} \delta^{2}\right) / \rho, \bar{\nu}=\left(\bar{n}_{1}+\bar{n}_{2} \delta+\bar{n}_{3} \delta^{2}\right) / \rho$, then

$$
\left(\begin{array}{ll}
m_{1}^{\prime} & n_{1}^{\prime} \\
\bar{m}_{2} & \bar{n}_{2} \\
\bar{m}_{3} & \bar{n}_{3}
\end{array}\right)=\left(\begin{array}{ll}
m_{1} & n_{1} \\
m_{2} & n_{2} \\
m_{3} & n_{3}
\end{array}\right) K
$$

and

$$
\bar{m}_{1} \equiv m_{1}^{\prime}(\bmod \rho), \quad \bar{n}_{1} \equiv n_{1}^{\prime}(\bmod \rho), \quad t_{21}=\left(m_{1}^{\prime}-\bar{m}_{1}\right) / \rho, \quad t_{31}=\left(n_{1}^{\prime}-\bar{n}_{1}\right) / \rho .
$$

5. The Bases of the Ideals of $2[\delta]$. In order to determine a method for finding a basis of $\Omega_{n}^{2}$ from a basis of $\Re_{n}$, it is first necessary to discuss the integral bases of the ideals of $2[\delta]$. The integral bases for the ideals in any cubic field were determined by Voronoi [14]. However, as this work is not easily accessible, we summarize some of his results here. There are a large number of cases, and this is one of the reasons that we will restrict our discussion, here and in the next section, to the case of the pure cubic field $2(\sqrt[3]{D})$, where $D=g_{1} g_{2}^{2}$ as in Section 1 . 
If $\mathfrak{p}$ is a prime ideal of $\stackrel{\mathcal{O}}{\sim}[\delta]$, then $\mathfrak{p}$ must divide $[p]$, where $p$ is some rational prime. It is well known (see, for example, Cassels [4]) that the principal ideals $[p]$ of (2) $[\delta]$ factor as follows. We have 7 cases.

(i) $p \mid g_{1}$. Here $[p]=\mathfrak{p}^{3}$, where $\mathfrak{p}=[p, \delta]$.

(ii) $p=3,3 \nmid D, D Z \pm 1(\bmod 9)$. Here $[3]=\mathfrak{p}^{3}$, where

$$
\mathfrak{p}=[3,1 \mp \delta] \text { when } D \equiv \pm 1(\bmod 3) \text {. }
$$

(iii) $p \mid g_{2}$. In this case $[p]=\mathfrak{p}^{3}$, where $\mathfrak{p}=\left[p, \delta^{2} / g_{2}\right]$.

(iv) $p=3, D \equiv \pm 1(\bmod 9)$. We have $[3]=r^{2} 5$, where

$$
\begin{aligned}
& r=\left[3,1 \mp \delta,\left(1 \pm \delta+\delta^{2}\right) / 3\right], \\
& \check{s}=\left[3,1 \mp \delta,\left(-2 \pm \delta+\delta^{2}\right) / 3\right],
\end{aligned}
$$

and the signs are determined from $D \equiv \pm 1(\bmod 9)$.

(v) $p \equiv-1(\bmod 3), p \nmid D$. Here $[p]=\mathfrak{p a}$, and

$$
\mathfrak{p}=[p, d-\delta], \quad \mathfrak{q}=\left[p, d^{2}+d \delta+\delta^{2}\right],
$$

where $d$ is the unique root of the congruence

$$
x^{3} \equiv D \quad(\bmod p) .
$$

(vi) $p \equiv 1(\bmod 3), p \nmid D,(D / p)_{3}=1$. In this case, $[p]=\mathfrak{p} \mathfrak{p}^{\prime} \mathfrak{p}^{\prime \prime}$, where

$$
\mathfrak{p}=[p, d-\delta], \quad \mathfrak{p}^{\prime}=\left[p, d^{\prime}-\delta\right], \quad \mathfrak{p}^{\prime \prime}=\left[p, d^{\prime \prime}-\delta\right],
$$

and $d, d^{\prime}, d^{\prime \prime}$ are the three distinct roots of the congruence (5.1).

(vii) $p \equiv 1(\bmod 3), p \nmid D,(D / p)_{3} \neq 1$. Here $[p]$ is a prime ideal of $\mathcal{Q}[\delta]$.

Let $c \in \stackrel{Z}{Z}$ be defined as being a solution of the system of congruences

$$
\begin{cases}x^{3} \equiv D & \left(\bmod \sigma^{2}\right) \\ 3 x^{2} \equiv 0 & (\bmod \sigma)\end{cases}
$$

Also, when the system of congruences

$$
\begin{cases}x^{3} \equiv D & \left(\bmod \sigma^{2} p^{i}\right) \\ x \equiv c & (\bmod \sigma)\end{cases}
$$

has a single root $\left(\bmod \sigma p^{i}\right)$, denote it by $d_{i}$; when $(5.3)$ has 3 roots $\left(\bmod \sigma p^{i}\right)$, denote them by $d_{i}, d_{i}^{\prime}, d_{i}^{\prime \prime}$. Since $3 d_{i}^{2} \neq 0\left(\bmod \sigma p^{i}\right)$, we may assume that none of $d_{i}, d_{i}^{\prime}, d_{i}^{\prime \prime}$ satisfies

$$
x^{3} \equiv D \quad\left(\bmod \sigma p^{i+1}\right)
$$

when $p \nmid D$.

Voronoi found the bases for the prime ideals given above, their powers, and certain products of their powers. We give his results for each of the cases (i) through (vi) below. We assume here that $i \in \mathscr{Z}$ and $i>0$, unless otherwise stated.

\section{Group A}

CASES

(i), (ii), $p \nmid \sigma$

(iii), $p \mid \sigma$
$I D E A L$ a

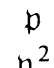

$p^{2}$

$\mathfrak{p}$

$p^{2}$
IDEAL BASIS OF a

$\left\{p,-d_{1}+\delta,\left(-c d_{1}-d_{1}^{2}+c \delta+\delta^{2}\right) / \sigma\right\}$

$\left\{p, p(-c+\delta),\left(d_{1}^{2}+d_{1} \delta+\delta^{2}\right) / \sigma\right\}$

$\left\{p,-c+\delta,\left(c^{2}+c \delta+\delta^{2}\right) / \sigma\right\}$

$\left\{p,-d_{1}+\delta, p\left(c^{2}+c \delta+\delta^{2}\right) / \sigma\right\}$ 
Notice that in each of these cases in Group A, we have $d_{1} \equiv c(\bmod \sigma), d_{1} \equiv D$ $(\bmod p)$ and $c \equiv D(\bmod \sigma) ;$ hence, $\mathfrak{p}$ has a basis of the form

$$
\left\{p,-D+\delta,\left(D^{2}+D \delta+\delta^{2}\right) / \sigma\right\} .
$$

Also, $\mathfrak{p}^{2}$ has a basis of the form

$$
\left\{p, p(-D+\delta),\left(D^{2}+D \delta+\delta^{2}\right) / \sigma\right\}
$$

when $p \nmid \sigma$ and $p^{2}$ has a basis of the form

$$
\left\{p,-D+\delta, p\left(D^{2}+D \delta+\delta^{2}\right) / \sigma\right\},
$$

when $p \mid \sigma$. Thus, if $t$ is either $p$ or $p^{2}$ here, then $t$ has a basis of the form

$$
\left\{P, P^{\prime}(-D+\delta), P^{\prime \prime}\left(D^{2}+D \delta+\delta^{2}\right) / \sigma\right\},
$$

and $\mathrm{t}^{2}=\left[P^{\prime} P^{\prime \prime}\right] \mathfrak{u}$, where $u$ has

$$
\left\{P,\left(P / P^{\prime} S\right)(-D+\delta),\left(S / P^{\prime \prime}\right)\left(D^{2}+D \delta+\delta^{2}\right) / \sigma\right\}
$$

as a basis and $S=\operatorname{gcd}(P, \sigma)$.

\section{GROUP B}

CASE IDEAL a IDEALBASIS OF a

(iv)

$$
\begin{aligned}
& \begin{array}{cl}
\mathrm{r} & \left\{3,-c+\delta,\left(c^{2}+c \delta+\delta^{2}\right) / \sigma\right\} \\
\mathrm{r}^{2 i} & \left\{3^{i}, 3^{i}(-c+\delta),\left(d_{i}^{2}+d_{i} \delta+\delta^{2}\right) / \sigma\right\} \\
\mathrm{r}^{2 i+1}(i \geqslant 0) & \left\{3^{i+1}, 3^{i}(-c+\delta),\left(d_{i}^{2}+d_{i} \delta+\delta^{2}\right) / \sigma\right\} \\
\mathfrak{S}^{i} & \left\{3^{i},-d_{i}+\delta,\left(-c d_{i}-d_{i}^{2}+c \delta+\delta^{2}\right) / \sigma\right\}
\end{array} \\
& \mathrm{r} \mathfrak{s} \quad\left\{3,-c+\delta, 3\left(c^{2}+c \delta+\delta^{2}\right) / \sigma\right\} \\
& r \mathfrak{s}^{i+1} \quad\left\{3^{i+1},-d_{i}+\delta, 3\left(-c d_{i}-d_{i}^{2}+c \delta+\delta^{2}\right) / \sigma\right\} \\
& \mathfrak{p}^{i} \quad\left\{p^{i},-d_{i}+\delta,\left(-c d_{i}-d_{i}^{2}+c \delta+\delta^{2}\right) / \sigma\right\} \\
& \mathrm{q}^{i} \quad\left\{p^{i}, p^{i}(-c+\delta),\left(d_{i}^{2}+d_{i} \delta+\delta^{2}\right) / \sigma\right\} \\
& \text { (vi) } \quad \mathfrak{p}^{i} \quad\left\{p^{i},-d_{i}+\delta,\left(-c d_{i}-d_{i}^{2}+c \delta+\delta^{2}\right) / \sigma\right\} \\
& \mathfrak{p}^{i}\left(\mathfrak{p}^{\prime}\right)^{i} \quad\left\{p^{i}, p^{i}(-c+\delta),\left(d_{i}^{\prime \prime 2}+d_{i}^{\prime \prime} \delta+\delta^{2}\right) / \sigma\right\} \\
& \mathfrak{p}^{i+j}\left(\mathfrak{p}^{\prime}\right)^{i} \quad\left\{p^{i+j}, p^{i}\left(-d_{j}+\delta\right),\left(\left(d_{i}^{\prime \prime 2}+d_{i}^{\prime \prime} \delta+\delta^{2}\right) / \sigma+p^{i} Q\right)\right\} .
\end{aligned}
$$

In case (vi) here, $Q$ is determined by the congruence

$$
Q\left(d_{j}-d_{i}^{\prime \prime}\right) / \sigma \equiv\left(d_{i}^{\prime \prime 3}-D\right) / \sigma^{2} p^{i}\left(\bmod p^{j}\right) .
$$

Naturally, there are similar results for $\left(\mathfrak{p}^{\prime \prime}\right)^{i},\left(\mathfrak{p}^{\prime}\right)^{i+j}\left(\mathfrak{p}^{\prime \prime}\right)^{i}$, etc. As we did not specify $\mathfrak{p}$ or $\mathfrak{p}^{\prime}$ here beyond saying that they were any two of the three ideals whose product is $[p]$, these other results can be easily deduced from those given here.

Each of the several ideals described in Groups A and B here can be represented by a basis of the general form

$$
\left\{P, P^{\prime}(t+\delta), P^{\prime \prime}\left(u+u^{\prime} \delta+\delta^{2}\right) / \sigma\right\},
$$

where $P, P^{\prime}, P^{\prime \prime}, t, u, u^{\prime} \in \mathscr{Z}, P$ is a prime power, $P^{\prime} P^{\prime \prime} \mid P$, and $P^{\prime \prime} \mid \sigma$. Further, it is a simple matter to verify that for each of these ideals we have

$$
\left\{\begin{array}{l}
u-t u^{\prime}+t^{2} \equiv 0 \quad\left(\bmod P / P^{\prime}\right) \\
u \equiv u^{\prime 2} \quad\left(\bmod \sigma P^{\prime} / P^{\prime \prime}\right) \\
u\left(u^{\prime}+t\right) \equiv D+t u^{\prime 2} \quad\left(\bmod \sigma P / P^{\prime \prime}\right)
\end{array}\right.
$$


That this representation of these ideals is essentially unique follows from the following lemma.

LEMMA 5.1. Let $\mathfrak{a}$ and $\mathfrak{b}$ be any two ideals of $2[\delta]$ such that a has a basis of the form $\left\{P, P^{\prime}(r+\delta), P^{\prime \prime}\left(s+s^{\prime} \delta+\delta^{2}\right) / \sigma\right\}$ and $\mathfrak{b}$ has a basis of the form $\{Q$, $\left.Q^{\prime}(u+\delta), Q^{\prime \prime}\left(v+v^{\prime} \delta+\delta^{2}\right) / \sigma\right\}$, where $P, P^{\prime}, P^{\prime \prime}, r, s^{\prime}, s, Q, Q^{\prime}, Q^{\prime \prime}, u, v^{\prime}, v \in$ Then $\mathfrak{b} \mid \mathfrak{a}$ if and only if $Q\left|P, Q^{\prime}\right| P^{\prime}, Q^{\prime \prime} \mid P^{\prime \prime}$ and

$$
\begin{aligned}
P^{\prime} r & \equiv P^{\prime} u \quad(\bmod Q), \\
P^{\prime \prime} s^{\prime} & \equiv P^{\prime \prime} v^{\prime} \quad\left(\bmod \sigma Q^{\prime}\right), \\
P^{\prime \prime} s & \equiv P^{\prime \prime} v+P^{\prime \prime} u\left(s^{\prime}-v^{\prime}\right) \quad(\bmod \sigma Q) .
\end{aligned}
$$

Proof. $\mathfrak{b} \mid \mathfrak{a}$ if and only if $\mathfrak{b} \supseteq a$; thus $\mathfrak{b} \mid \mathfrak{a}$ if and only if each of $P, P^{\prime}(r+\delta)$, $P^{\prime \prime}\left(s+s^{\prime} \delta+\delta^{2}\right) / \sigma$ is contained in $\mathfrak{b}$. If

$$
M_{1}=\left(\begin{array}{ccc}
\sigma P & \sigma P^{\prime} r & P^{\prime \prime} s \\
0 & \sigma P^{\prime} & P^{\prime \prime} s^{\prime} \\
0 & 0 & P^{\prime \prime}
\end{array}\right), \quad M_{2}=\left(\begin{array}{ccc}
\sigma Q & \sigma Q^{\prime} u & Q^{\prime \prime} v \\
0 & \sigma Q^{\prime} & Q^{\prime \prime} v^{\prime} \\
0 & 0 & Q^{\prime \prime}
\end{array}\right)
$$

then $P, P^{\prime}(r+\delta), P^{\prime \prime}\left(s+s^{\prime} \delta+\delta^{2}\right) / \sigma \in \mathfrak{b}$ if and only if there exists a matrix $X=\left(x_{i j}\right)_{3 \times 3}$ where $x_{i j} \in{ }^{\prime}(i=1,2,3 ; j=1,2,3)$ such that $M_{1}=M_{2} X$. The result follows easily on multiplying $M_{2}$ by $X$, equating the product to $M_{1}$ and attempting to solve for the rational integers $x_{i j}$.

Corollary. Let $\mathfrak{a}$ and $\mathfrak{b}$ be given as above, and suppose that $P\left|P^{\prime}, P^{\prime \prime}\right| \sigma$, $Q, P, Q^{\prime}, P^{\prime}, Q^{\prime \prime}, P^{\prime \prime}>0$; then $\mathfrak{a}=\mathfrak{b}$ if and only if

$$
Q=P, \quad Q^{\prime}=P^{\prime}, \quad Q^{\prime \prime}=P^{\prime \prime}
$$

and

$$
\begin{aligned}
r & \equiv u \quad\left(\bmod P / P^{\prime}\right), \\
s^{\prime} & \equiv v^{\prime} \quad\left(\bmod \sigma P^{\prime} / P^{\prime \prime}\right), \\
s & \equiv v+u\left(s^{\prime}-v^{\prime}\right) \quad\left(\bmod \sigma P / P^{\prime \prime}\right) .
\end{aligned}
$$

Proof. Follows easily from the lemma and the fact that $a=\mathfrak{b}$ if and only if $\mathfrak{a} \mid \mathfrak{b}$ and $\mathfrak{b} \mid a$.

From these results we are now able to deduce the following lemma.

LEMMA 5.2. Let $\mathfrak{a}$ and $\mathfrak{b}$ be ideals of $2[\delta]$ such that a has a basis of the form $\left\{P, P^{\prime}(r+\delta), P^{\prime \prime}\left(s+s^{\prime} \delta+\delta^{2}\right) / \sigma\right\}$ and $\mathfrak{b}$ has a basis of the form $\left\{P, P^{\prime}(t+\delta), P^{\prime \prime}\left(u+u^{\prime} \delta+\delta^{2}\right) / \sigma\right\}$, where $r, s, s^{\prime}, t, u, u^{\prime}, P, P^{\prime}, P^{\prime \prime} \in \mathcal{Z}$, $P^{\prime} P^{\prime \prime}\left|P, P^{\prime \prime}\right| \sigma$ and $t, u, u^{\prime}$ satisfy the congruences (5.4). If $\mathfrak{a}=\mathfrak{b}$, then $r, s^{\prime}, s, P, P^{\prime}$, $P^{\prime \prime}$ must satisfy the congruences

$$
\left\{\begin{array}{l}
r^{3} \equiv-D \quad\left(\bmod P / P^{\prime}\right) \\
s^{\prime 3} \equiv D \quad\left(\bmod \sigma P^{\prime} / P^{\prime \prime}\right) \\
s-r s^{\prime}+r^{2} \equiv 0 \quad\left(\bmod P / P^{\prime}\right) \\
s \equiv s^{\prime 2} \quad\left(\bmod \sigma P^{\prime} / P^{\prime \prime}\right) \\
s\left(s^{\prime}+r\right) \equiv D+r s^{\prime 2} \quad\left(\bmod \sigma P / P^{\prime \prime}\right)
\end{array}\right.
$$


Proof. Since $a=\mathfrak{b}$, we must have

$$
\begin{aligned}
& r \equiv t \quad\left(\bmod P / P^{\prime}\right), \quad s^{\prime} \equiv u^{\prime} \quad\left(\bmod \sigma P^{\prime} / P^{\prime \prime}\right), \\
& s \equiv u+\left(s^{\prime}-u\right) t \quad\left(\bmod \sigma P / P^{\prime \prime}\right)
\end{aligned}
$$

from the corollary of Lemma 5.1. Using these results together with the congruences (5.4), it is a routine matter to deduce the last three congruences of (5.5). The first two can be easily deduced from these last three.

Voronoi [14, Section 44] also proved the theorem which follows.

THEOREM 5.3. Suppose $\mathfrak{a}$ and $\mathfrak{b}$ are ideals of $\mathcal{2}[\delta]$ such that a has a basis of the form $\left\{P_{1}, P_{1}^{\prime}\left(r_{1}+\delta\right), P_{1}^{\prime \prime}\left(s_{1}+s_{1}^{\prime} \delta+\delta^{2}\right) / \sigma\right\}$ and $\mathfrak{b}$ has a basis of the form $\left\{P_{2}\right.$, $\left.P_{2}^{\prime}\left(r_{2}+\delta\right), P_{2}^{\prime \prime}\left(s_{2}+s_{2}^{\prime} \delta+\delta^{2}\right) / \sigma\right\}$, where $r_{i}, s_{i}, s_{i}^{\prime}, P_{i}, P_{i}^{\prime}, P_{i}^{\prime \prime} \in \mathcal{Z}, P_{i}^{\prime} P_{i}^{\prime \prime}\left|P_{i}, P_{i}^{\prime \prime}\right| \sigma$, $(i=1,2)$. If $\operatorname{gcd}\left(P_{1}, P_{2}\right)=1$ and $\mathfrak{c}=\mathfrak{a} \mathfrak{b}$, then $c$ has a basis $\left\{P_{3}, P_{3}^{\prime}\left(r_{3}+\delta\right)\right.$, $\left.P_{3}^{\prime \prime}\left(s_{3}+s_{3}^{\prime} \delta+\delta^{2}\right) / \sigma\right\}$, where

$$
\begin{gathered}
P_{3}=P_{1} P_{2}, \quad P_{3}^{\prime}=P_{1}^{\prime} P_{2}^{\prime}, \quad P_{3}^{\prime \prime}=P_{1}^{\prime \prime} P_{2}^{\prime \prime}, \\
\left\{\begin{array} { l l } 
{ r _ { 3 } \equiv r _ { 1 } \quad ( \operatorname { m o d } P _ { 1 } / P _ { 1 } ^ { \prime } ) , } \\
{ r _ { 3 } \equiv r _ { 2 } \quad ( \operatorname { m o d } P _ { 2 } / P _ { 2 } ^ { \prime } ) , }
\end{array} \left\{\begin{array}{ll}
s_{3}^{\prime} \equiv s_{1}^{\prime} & \left(\bmod \sigma P_{1}^{\prime} / P_{3}^{\prime \prime}\right), \\
s_{3}^{\prime} \equiv s_{2}^{\prime} & \left(\bmod \sigma P_{2}^{\prime} / P_{3}^{\prime \prime}\right),
\end{array}\right.\right. \\
\begin{cases}s_{3} \equiv s_{1}+r_{1}\left(s_{3}^{\prime}-s_{1}^{\prime}\right) & \left(\bmod \sigma P_{1} / P_{3}^{\prime \prime}\right), \\
s_{3} \equiv s_{2}+r_{2}\left(s_{3}^{\prime}-s_{2}^{\prime}\right) & \left(\bmod \sigma P_{2} / P_{3}^{\prime \prime}\right)\end{cases}
\end{gathered}
$$

Let $a$ be any ideal of $2[\delta]$ such that $[p]$ does not divide a for any rational prime $p$. Then, since a can be written as a product of powers of distinct prime ideals, we have

$$
a=t_{1} t_{2} t_{3} \cdots t_{k}
$$

where the $\mathrm{t}_{i}(i=1,2,3, \ldots, k)$ are ideals of Group A or Group B above. Since $\mathrm{t}_{i}$ $(i=1,2,3, \ldots, k)$ has a basis

$$
\left\{p_{i}^{n_{i}}, p_{i}^{m_{i}}\left(t_{i}+\delta\right), p_{i}^{l_{i}}\left(u_{i}+u_{i}^{\prime} \delta+\delta^{2}\right) / \sigma\right\}
$$

where $p_{i}^{l_{i}} \mid \sigma, n_{i} \geqslant m_{i}+l_{i}, m_{i} l_{i}=0$, and $\operatorname{gcd}\left(p_{i}, p_{j}\right)=1$ for $i \neq j$, we see by Theorem 5.3 that $a$ has a basis of the form

$$
\left\{P, P^{\prime}(r+\delta), P^{\prime \prime}\left(s+s^{\prime} \delta+\delta^{2}\right) / \sigma\right\}
$$

where

$$
P=\prod_{i=1}^{k} p_{i}^{n_{1}}, \quad P^{\prime}=\prod_{i=1}^{k} p_{i}^{m_{i}}, \quad P^{\prime \prime}=\prod_{i=1}^{k} p_{i}^{l_{i}},
$$

$r, s, s^{\prime} \in \mathscr{Z}, P^{\prime} P^{\prime \prime}\left|P, P^{\prime \prime}\right| \sigma$, and $\operatorname{gcd}\left(P, P^{\prime}, P^{\prime \prime}\right)=1$. Note that $P=L($ a $)$. It is also true that the congruences (5.5) are satisfied. This follows from the theorem below.

THEOREM 5.4. Let the ideals $\mathfrak{a}, \mathfrak{b}, \mathfrak{c}$ be defined as in Theorem 5.3. If $r_{i}, s_{i}^{\prime}, s_{i}, P_{i}, P_{i}^{\prime}, P_{i}^{\prime \prime}$ satisfy the congruences (5.5) when $i=1$ and $i=2$, then $r_{3}, s_{3}^{\prime}, s_{3}, P_{3}, P_{3}^{\prime}, P_{3}^{\prime \prime}$, also satisfy (5.5).

Proof. We will only show that

$$
s_{3}\left(s_{3}^{\prime}+r_{3}\right) \equiv D+r_{3} s_{3}^{\prime 2}\left(\bmod \sigma P_{3} / P_{3}^{\prime \prime}\right)
$$


The remaining results can be derived in a somewhat similar fashion. We first note that the congruences of Theorem 5.3 must be satisfied; hence

$$
\begin{aligned}
& r_{3}=r_{1}+k_{1} P_{1} / P_{1}^{\prime}, \quad s_{3}^{\prime}=s_{1}^{\prime}+k_{2} \sigma P_{1}^{\prime} / P_{3}^{\prime \prime}, \\
& s_{3} \equiv s_{1}+\sigma r_{1} k_{2} P_{1}^{\prime} / P_{3}^{\prime \prime} \quad\left(\bmod \sigma P_{1} / P_{3}^{\prime \prime}\right),
\end{aligned}
$$

where $k_{1}, k_{2} \in$. From these results, we get

$$
\begin{aligned}
\left(r_{3}+s_{3}^{\prime}\right) s_{3} \equiv & \left(r_{1}+s_{1}^{\prime}+k_{1} P_{1} / P_{1}^{\prime}+k_{2} \sigma P_{1}^{\prime} / P_{3}^{\prime \prime}\right)\left(s_{1}+\sigma r_{1} k_{2} P_{1}^{\prime} / P_{3}^{\prime \prime}\right) \\
\equiv & \left(r_{1}+s_{1}^{\prime}\right) s_{1}+\sigma\left(r_{1}+s_{1}^{\prime}\right) r_{1} k_{2} P_{1}^{\prime} / P_{2}^{\prime \prime} \\
& +k_{1} s_{1} P_{1} / P_{1}^{\prime}+\sigma k_{2} s_{1} P_{1}^{\prime} / P_{3}^{\prime \prime}+k_{2}^{2} r_{1}\left(\sigma P_{1}^{\prime} / P_{3}^{\prime \prime}\right)^{2} \quad\left(\bmod \sigma P_{1} / P_{3}^{\prime \prime}\right)
\end{aligned}
$$

and

$$
\begin{aligned}
D+r_{3} s_{3}^{\prime 2} \equiv & D+r_{1} s_{1}^{\prime 2}+2 \sigma r_{1} s_{1}^{\prime} k_{2} P_{1}^{\prime} / P_{3}^{\prime \prime}+k_{2}^{2} r_{1}\left(\sigma P_{1}^{\prime} / P_{3}^{\prime \prime}\right)^{2} \\
& +k_{1} s_{1}^{\prime 2} P_{1} / P_{1}^{\prime} \quad\left(\bmod \sigma P_{1} / P_{3}^{\prime \prime}\right)
\end{aligned}
$$

Since

$$
\begin{aligned}
\left(r_{1}+s_{1}^{\prime}\right) s_{1} & \equiv D+r_{1} s_{1}^{\prime 2} \quad\left(\bmod \sigma P_{1} / P_{1}^{\prime \prime}\right), \\
s_{1}^{\prime 2} & \equiv s \quad\left(\bmod \sigma P_{1}^{\prime} / P_{1}^{\prime \prime}\right), \\
s_{1} & \equiv r_{1} s_{1}^{\prime}-r_{1}^{2} \quad\left(\bmod P_{1} / P_{1}^{\prime}\right),
\end{aligned}
$$

we get $\left(r_{3}+s_{3}^{\prime}\right) s_{3} \equiv D+r_{3} s_{3}^{\prime 2}\left(\bmod \sigma P_{1} / P_{3}^{\prime \prime}\right)$. Similarly, we also get $\left(r_{3}+s_{3}^{\prime}\right) s_{3} \equiv D$ $+r_{3} s_{3}^{\prime 2}\left(\bmod \sigma P_{2} / P_{3}^{\prime \prime}\right)$. Thus, since $\operatorname{gcd}\left(P_{1}, P_{2}\right)=1$, we see that $(5.8)$ must follow.

COROllary 5.4.1. For a basis (5.7) of an ideal of $\stackrel{\mathcal{\sim}}{\sim}[\delta]$ we must have $r, s, s^{\prime}, P, P^{\prime}, P^{\prime \prime}$ satisfying (5.5).

COROllary 5.4.2. For a basis (5.7) of an ideal of $\stackrel{2}{\sim}[\delta]$ we must have

$$
s^{2}+s s^{\prime 2} \equiv 2 s^{\prime} D+r\left(s^{\prime 3}-D\right) \quad\left(\bmod \sigma P / P^{\prime \prime}\right) .
$$

Proof. From the theorem we know that the congruences (5.5) must hold; hence

$$
\left(s-s^{\prime 2}\right)\left(s-r s^{\prime}+r^{2}\right) \equiv 0 \quad\left(\bmod \sigma P / P^{\prime \prime}\right)
$$

and

$$
\left(s^{\prime}+r\right) s \equiv D+r s^{\prime 2} \quad\left(\bmod \sigma P / P^{\prime \prime}\right) .
$$

From the first of these congruences, we get

$$
s^{2}+s^{\prime} s \equiv s s^{\prime} r-s r^{2}+2 s^{\prime} s-s^{\prime 3} r+s^{\prime 2} r^{2} \quad\left(\bmod \sigma P / P^{\prime \prime}\right) .
$$

Using

$$
s r \equiv D+r s^{\prime 2}-s s^{\prime} \quad\left(\bmod \sigma P / P^{\prime \prime}\right)
$$

to substitute for $s r$ in the first term of the above, we get

$$
\begin{aligned}
s^{2}+s^{\prime 2} s & \equiv s^{\prime} D+s^{\prime 2} r^{2}+s\left(s^{\prime}+r\right)\left(s^{\prime}-r\right) \\
& \equiv 2 s^{\prime} D+r\left(s^{\prime 3}-D\right) \quad\left(\bmod \sigma P / P^{\prime \prime}\right)
\end{aligned}
$$

We also require two simple lemmas.

Lemma 5.5. Let $D \equiv \pm 1(\bmod 9)$, and let a be an ideal of $2[\delta]$ such that a has a basis of the form (5.7). If $3 \mid P$ and $3 \nmid P^{\prime \prime}$, we have $\mathrm{r} \mid \mathrm{a}$ when $s^{2}+2 s \equiv 3(\bmod 9)$ and $\mathfrak{s} \mid \mathfrak{a}$ when $s^{\prime 2}+2 s \equiv 6(\bmod 9)$. Here $\mathrm{r}$ and $\mathfrak{s}$ are the ideals of case (iv) in Group B. 
Proof. Clearly, since $3 \nmid P^{\prime \prime}$, we see that $\mathrm{r} \backsim \nmid$ a. However, since $3 \mid P$, one of $\mathrm{r}$ or $\mathfrak{s}$ must be a divisor of $a$. If $r \mid a$, by Lemma 5.1, we have

$$
s^{\prime} \equiv c \quad(\bmod \sigma), \quad s \equiv c^{2}+\left(s^{\prime}-c\right)(-c) \quad(\bmod 3 \sigma) .
$$

Since $3 \mid \sigma$, we see that $s^{\prime 2}+2 s \equiv\left(s^{\prime}-c\right)^{2}+3 c^{2} \equiv 3(\bmod 9)$. If $\mathfrak{s} \mid \mathfrak{a}$, by Lemma 5.1, we have

$$
s^{\prime} \equiv c \quad(\bmod \sigma), \quad s \equiv-c d_{1}-d_{1}^{2}+\left(s^{\prime}-c\right)\left(-d_{1}\right) \quad(\bmod 3 \sigma) .
$$

Hence, $s \equiv-d_{1}^{2}-d_{1} s^{\prime}(\bmod 9)$ and $s^{\prime 2}+2 s \equiv\left(s^{\prime}-d_{1}\right)^{2}-3 d_{1}^{2} \equiv 6(\bmod 9)$.

If $a$ is an ideal of ${ }^{2}[\delta]$ such that a has a basis of the form (5.7), define $3^{\iota^{\prime \prime}}$ for a to be the exact power of 3 that divides $\operatorname{gcd}(P, \sigma)$ and define $3^{\prime}$ to be the exact power of 3 that divides $\sigma / P^{\prime \prime}$. Since $\sigma$ is always square free, we see that both $\iota_{0}$ and $\iota_{1}$ are either 0 or 1 .

LEMma 5.6. Let a be an ideal of $\cong[\delta]$ such that a has a basis of the form (5.7) and

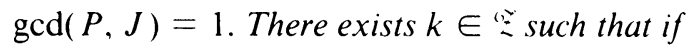

$$
v^{\prime}=s^{\prime}+\sigma k P^{\prime} / P^{\prime \prime}, \quad v=s+k r \sigma P^{\prime} / P^{\prime \prime},
$$

then a also has

$$
\left\{P, P^{\prime}(r+\delta), P^{\prime \prime}\left(v+v^{\prime} \delta+\delta^{2}\right) / \sigma\right\}
$$

as a basis, $3^{\iota^{\prime}} \mid v^{\prime 2}+2 v$, and $\operatorname{gcd}\left(v^{\prime 2}+2 v, 3^{\iota_{1}+\iota^{\prime}} P\right)=3^{\iota^{1}}$.

Proof. Select $k$ such that, for each distinct prime $q$ which divides $3^{\iota^{\prime}} P$ but does not divide $\sigma P^{\prime} / P^{\prime \prime}$, we have

$$
\left(s^{\prime}+r+k \sigma P^{\prime} / P^{\prime \prime}\right)^{2} \not 3 r^{2} \quad(\bmod q) .
$$

Certainly, we can find such a $k$. By Lemma 5.1, it is clear that (5.10) is a basis of a. Hence, by Corollary 5.3.1, we must have

$$
v^{\prime 3} \equiv D, \quad v \equiv v^{\prime 2} \quad\left(\bmod \sigma P^{\prime} / P^{\prime \prime}\right) .
$$

Let $p$ be any prime such that $p \mid 3^{\iota_{1}} P$. If $p \mid \sigma P^{\prime} / P^{\prime \prime}$, then $v^{\prime 3} \equiv D(\bmod p)$. If $p \neq 3$, we see that if $p \mid v^{\prime 2}+2 v$, then $p \mid D$, which is impossible. If $p=3$, then $\iota=1$. Since $3 \mid \sigma P^{\prime} / P^{\prime \prime}$, we have $3 \mid P^{\prime}$ or $3 \mid \sigma / P^{\prime \prime}$. If $3 \mid P^{\prime}$, then $3 \nmid P^{\prime \prime}$, and therefore $3 \mid \sigma / P^{\prime \prime}$. Thus $\iota_{1}=1$ and $3 \nmid P^{\prime \prime}$. Hence $3^{\iota_{1}} \mid\left(v^{\prime 2}+2 v\right)$ and $9 \nmid v^{\prime 2}+2 v$. Now suppose that $p \nmid \sigma P^{\prime} / P^{\prime \prime}$. If $p=3^{\iota_{0}}$ and $\iota_{0}=1$, then $3\left|P^{\prime \prime}, 3 \nmid P^{\prime}, 3\right| P$ and $3 \mid P / P^{\prime}$. Since

$$
v^{\prime 2}+2 v=\left(s^{\prime}+r+k \sigma P^{\prime} / P^{\prime \prime}\right)^{2}+2\left(s-r s^{\prime}+r^{2}\right)-3 r^{2}
$$

and $s-r s^{\prime}+r^{2} \equiv 0\left(\bmod P / P^{\prime}\right)$, we see that $p \nmid\left(v^{\prime 2}+2 v\right)$ by construction of $k$. It follows that $\left(\left(v^{\prime 2}+2 v\right) / 3^{\iota}, 3^{\prime}>P\right)=1$.

Since $R$ in Section 4 is similar to $R_{1}$ and $R_{1}$ corresponds to the ideal $\mathfrak{o}$, we know by Lemma 2.2 that $R$ corresponds to some ideal a of $\mathcal{2}[\delta]$. We may assume with no loss of generality that a has a basis of the form (5.7). It follows (Lemma 5.1) that a canonical basis of the form (4.3) of $R$ must have

$$
\left\{\begin{array}{l}
n^{\prime \prime}=1, \quad f=b= \pm \sigma P^{\prime} / P^{\prime \prime}, \quad \rho=\sigma P / P^{\prime \prime}, \quad f \mid m \\
m / f \equiv r \quad(\bmod \rho / f), \\
n^{\prime} \equiv s \quad(\bmod f) \\
n \equiv s+r\left(n^{\prime}-s\right) \quad(\bmod \rho) .
\end{array}\right.
$$


Also, if $q$ is any prime such that $q \mid P^{\prime \prime}$, then $q \mid P$ and $q \nmid P^{\prime}$; hence $q \mid \sigma$ and $q \nmid f$. Further, if $q \mid \sigma$ and $q \nmid f$, then $q \mid P^{\prime \prime}$. Thus, since $P^{\prime \prime} \mid \sigma$ and $\sigma$ is square-free, we have

$$
P^{\prime \prime}=\sigma / \operatorname{gcd}(\sigma, f) \text {. }
$$

If $(h)=h_{i}$ for some $i$, then $|f|=\left|e_{i}\right|, \rho=\sigma_{i}$. Hence, $P \leqslant \sigma_{i}, P^{\prime} \leqslant\left|e_{i}\right|, P^{\prime \prime} \leqslant \sigma$ and

$$
P<3 D, \quad P^{\prime}<3 D, \quad P^{\prime \prime}<\sigma
$$

by (6.2) of [18]. Also, we note that since $n^{\prime 3} \equiv D(\bmod f)$, we must have

$$
\operatorname{gcd}\left(f, n^{\prime}\right) \mid D \text {. }
$$

Let $\Omega_{j}$ and $R_{k}$ be any two reduced lattices such that $R_{j} \sim R_{k} \sim R_{R}$. We may assume that $\left(R, R_{j}\right.$ and $R_{k}$ have canonical bases

$$
\begin{gathered}
\left\{1,\left|e_{j}\right|\left(m_{j}+\delta\right) / \sigma_{j},\left(n_{j}+n_{j}^{\prime} \delta+\delta\right) / \sigma_{j}\right\}, \\
\left\{1,\left|e_{k}\right|\left(m_{k}+\delta\right) / \sigma_{k},\left(n_{k}+n_{k}^{\prime} \delta+\delta^{2}\right) / \sigma_{k}\right\},
\end{gathered}
$$

respectively. If $a_{j}$ is the ideal corresponding to $R_{j}$ and $a_{k}$ is the ideal corresponding to $s_{h}$, then

$$
L\left(a_{j}\right)=\sigma_{j} / \operatorname{gcd}\left(\sigma, e_{j}\right), \quad L\left(a_{k}\right)=\sigma_{k} / \operatorname{gcd}\left(\sigma, e_{k}\right)
$$

by (5.11) and (5.12).

If $\operatorname{gcd}\left(L\left(a_{j}\right), L\left(a_{k}\right)\right)=1$, by Theorem (5.3) we have

$$
\left(R _ { j } \left(R_{k}=\left\langle 1,|f|(M+\delta) / \rho,\left(N+N^{\prime} \delta+\delta^{2}\right) / \rho\right\rangle,\right.\right.
$$

where $f=e_{j} e_{k} / \sigma, \rho=\sigma_{j} \sigma_{k} / \sigma$,

$$
\begin{gathered}
\left\{\begin{array} { l } 
{ M \equiv m _ { j } \quad ( \operatorname { m o d } \sigma _ { j } / e _ { j } ) , } \\
{ M \equiv m _ { k } \quad ( \operatorname { m o d } \sigma _ { k } / e _ { k } ) , }
\end{array} \quad \left\{\begin{array}{l}
N^{\prime} \equiv n_{j}^{\prime} \quad\left(\bmod \operatorname{gcd}\left(\sigma, e_{k}\right) e_{j} / \sigma\right), \\
N^{\prime} \equiv n_{k}^{\prime} \quad\left(\bmod \operatorname{gcd}\left(\sigma, e_{j}\right) e_{k} / \sigma\right) .
\end{array}\right.\right. \\
\left\{\begin{array}{l}
N \equiv n_{j}+m_{j}\left(N^{\prime}-n_{j}^{\prime}\right) \quad\left(\bmod \operatorname{gcd}\left(\sigma, e_{k}\right) \sigma_{j} / \sigma\right), \\
N \equiv n_{k}+m_{k}\left(N^{\prime}-n_{k}^{\prime}\right) \quad\left(\bmod \operatorname{gcd}\left(\sigma, e_{j}\right) \sigma_{k} / \sigma\right) .
\end{array}\right.
\end{gathered}
$$

In the next section we show how, given $R_{i}$, to find $\Omega_{i}^{2}$.

6. Determination of $\Re_{i}^{2}$. Let

$$
\Re_{i}=\left\langle 1,\left(m_{1}+m_{2} \delta+m_{3} \delta^{2}\right) / \sigma_{i},\left(n_{1}+n_{2} \delta+n_{3} \delta^{2}\right) / \sigma_{i}\right\rangle,
$$

where, as usual, $m_{1}, m_{2}, m_{3}, n_{1}, n_{2}, n_{3}, \sigma_{i} \in \mathscr{Z}, \quad \sigma_{i}>0, \quad e_{i}=m_{2} n_{3}-m_{3} n_{2}$, $\operatorname{gcd}\left(m_{1}, m_{2}, m_{3}, n_{1}, n_{2}, n_{3}, \sigma_{i}\right)=1$. Let

$$
\Re_{i}^{2}=\left\langle 1,\left(M_{1}+M_{2} \delta+M_{3} \delta^{2}\right) / \rho,\left(N_{1}+N_{2} \delta+N_{2} \delta^{2}\right) / \rho\right\rangle,
$$

where $M_{1}, M_{2}, M_{3}, N_{1}, N_{2}, N_{3}, \rho \in \mathcal{Z}, \rho>0, f=M_{2} N_{3}-M_{3} N_{2}$,

$$
\operatorname{gcd}\left(M_{1}, M_{2}, M_{3}, N_{1}, N_{2}, N_{3}, \rho\right)=1 \text {. }
$$

In this section, we will describe how to find $\rho, M_{1}, M_{2}, M_{3}, N_{1}, N_{2}, N_{3}$ from the values of $m_{j}, n_{j}(j=1,2,3)$ and $\sigma_{i}$ above. In order to do this, we must first show how to find a basis of $a^{2}$, given a basis of the type (5.7) of $a$.

From (5.6), we can write $a=a_{1} a_{2}$, where all the ideals dividing $a_{1}$ are from Group A and all the ideals dividing $a_{2}$ are from Group B. By referring to the bases of the ideals of Group $A$ and Theorem 5.3, we deduce that $\mathfrak{a}_{1}^{2}=\left[P_{1}^{\prime} P_{1}^{\prime \prime}\right] \mathfrak{b}_{1}$, where 
$\mathfrak{b}_{1}$ has basis $\left\{P_{1},\left(P_{1} / P_{1}^{\prime} S\right)(-D+\delta),\left(S / P_{1}^{\prime \prime}\right)\left(D^{2}+D \delta+\delta^{2}\right) / \sigma\right\}$. Here $P_{1}=$ $\operatorname{gcd}(P, J), P_{1}^{\prime}=\operatorname{gcd}\left(P^{\prime}, J\right), P_{1}^{\prime \prime}=\operatorname{gcd}\left(P^{\prime \prime}, J\right), S=\operatorname{gcd}\left(\sigma, P_{1}\right)$, and $a_{1}$ has basis $\left\{P_{1}, P_{1}^{\prime}(-D+\delta), P_{1}^{\prime \prime}\left(D^{2}+D \delta+\delta^{2}\right) / \sigma\right\}$.

If $P_{2}=P / P_{1}, P_{2}^{\prime}=P^{\prime} / P_{1}^{\prime}, P_{2}^{\prime \prime}=P^{\prime \prime} / P_{2}^{\prime \prime}$, we have a basis $\left\{P_{2}, P_{2}^{\prime}(r+\delta)\right.$, $\left.P_{2}^{\prime \prime}\left(s+s^{\prime} \delta+\delta^{2}\right) / \sigma\right\}$ of $a_{2}$.

We must now determine a basis for $a_{2}^{2}$. We first calculate $\iota_{0}, \iota_{1}$ for $a_{2}$ and then calculate $\iota_{2}$ and $\iota_{3}$ as below

$$
\begin{aligned}
& \iota_{2}= \begin{cases}1 & \text { when } \iota_{0}=1, \quad \text { and either } 3 \mid P_{2}^{\prime \prime} \text { or } s^{\prime 2}+2 s \equiv 6(\bmod 9), \\
0 & \text { otherwise. }\end{cases} \\
& \iota_{3}= \begin{cases}1 & \text { when } \iota_{0}=1, \\
0 & \text { otherwise. }\end{cases}
\end{aligned}
$$

By Lemmas 5.1 and 5.5, the bases of the ideals of case (iv) in Group B and Theorem 5.3 , it follows that we can have 6 possible cases.

(1) $D Z \pm 1(\bmod 9)$. In this case $\iota_{0}=\iota_{1}=\iota_{2}=\iota_{3}=0$. In the remaining cases, we assume that $D \equiv \pm 1(\bmod 9)$.

(2) $r \nmid a_{2}, \mathfrak{s} \nmid a_{2}$. Here $3 \nmid P_{2}$ and $\iota_{0}=\iota_{1}=\iota_{2}=\iota_{3}=0$.

(3) $\mathrm{r} \mathfrak{s} \mid \mathfrak{a}_{2}$. We have $3\left|P_{2}, 3 \nmid P_{2}^{\prime}, 3\right| P_{2}^{\prime \prime}$ and $\iota_{0}=1, \iota_{1}=0, \iota_{2}=1, \iota_{3}=0$.

(4) $\mathfrak{s} \mid \mathrm{a}_{2}, \mathrm{r} \nmid \mathrm{a}_{2}$. We have $3 \mid P_{2}, 3 \nmid P_{2}^{\prime}, 3 \nmid P_{2}^{\prime \prime}, s^{\prime 2}+2 s \equiv 6(\bmod 9)$ and $\iota_{0}=1$, $\iota_{1}=1, \iota_{2}=1, \iota_{3}=0$.

(5) $\mathrm{r}^{2 j} \mid \mathfrak{a}_{2}, \mathrm{r}^{2 j+1} \nmid \mathfrak{a}_{2}, j>0, \mathfrak{s} \nmid \mathfrak{a}_{2}$. We have $3 \mid P_{2}, 3 \nmid P_{2}^{\prime \prime}, 3 \nmid P_{2} / P_{2}^{\prime}, s^{\prime 2}+2 s \equiv 3$ $(\bmod 9)$ and $\iota_{0}=1, \iota_{1}=1, \iota_{2}=0, \iota_{3}=0$.

(6) $\mathrm{r}^{2 j+1} \mid \mathrm{a}_{2}, \mathrm{r}^{2 j+2} \nmid \mathrm{a}_{2}, j \geqslant 0, \mathfrak{s} \nmid \mathrm{a}_{2}$. We have $3\left|P_{2}, 3 \nmid P_{2}^{\prime \prime}, 9 \nmid P_{2} / P_{2}^{\prime}, 3\right| P_{2} / P_{2}^{\prime}$, $s^{\prime 2}+2 s \equiv 3(\bmod 9)$ and $\iota_{0}=1, \iota_{1}=1, \iota_{2}=0, \iota_{3}=1$.

Note that in all of these cases, we have

$$
3^{\iota_{3}} \mid\left(P_{2} / P_{2}^{\prime} P_{2}^{\prime \prime}\right), \quad \operatorname{gcd}\left(3^{1-\iota_{2}}, P_{2} / 3^{\iota_{3}} P_{2}^{\prime}\right)=1 .
$$

By Theorem 5.3 and the bases of the ideals in Group B, we have $\mathfrak{a}_{2}^{2}=\left[P_{2}^{\prime \prime}\right] \mathfrak{b}_{2}$, where $\mathfrak{b}_{2}$ is an ideal with basis

$$
\left\{P_{3}, P_{3}^{\prime}(U+\delta),\left(V+V^{\prime} \delta+\delta^{2}\right) / \sigma\right\}
$$

and $P_{3}=P_{2}^{2} / 3^{\iota_{3}} P_{2}^{\prime \prime}, P_{3}^{\prime}=3{ }^{\iota_{3}} P_{2}^{\prime 2}$. We need now to find the values of $U, V^{\prime}$ and $V$ in (6.2). We require the following lemma.

Lemma 6.1. Let $D \equiv \pm 1(\bmod 9)$. If a is any ideal of $2[\delta]$ with basis of the form (5.7) such that $\mathfrak{g} \mid \mathfrak{a}$, then

$$
r^{3} \equiv-D \quad\left(\bmod 3 P / P^{\prime}\right)
$$

If $\mathfrak{s} \nmid \mathfrak{a}$ and $\mathfrak{r} \mid \mathfrak{a}$, then

$$
s^{\prime 3} \equiv D \quad\left(\bmod 3 \sigma P^{\prime} / P^{\prime \prime}\right)
$$

Proof. Certainly, $r^{3} \equiv-D\left(\bmod P / P^{\prime}\right)$ and since $\xi \mid a$, we must have $3 \nmid P^{\prime}$. Also, if $3^{k} \| P$, then $k \geqslant 1$. Let $d_{j}$ be a root of (5.3) with $p=3$; then $d_{j}^{3} \equiv D \bmod \left(\sigma^{2} 3^{j}\right)$ and since $3 \nmid P^{\prime}$, by Lemma 5.1 , we have $r \equiv d_{j}\left(\bmod 3^{k}\right)$, where $j=k$ or $k-1$. Thus, $r^{3} \equiv d_{j}^{3}\left(\bmod 3^{k+1}\right)$ and $r^{3} \equiv D\left(\bmod 3 P / P^{\prime}\right)$. The second result follows by similar reasoning. 
Since $P_{2} P_{2}^{\prime}(r+\delta) \in a_{2}^{2}$, we have

$$
r \equiv U \quad\left(\bmod P_{3} P_{2}^{\prime \prime} / P_{2} P_{2}^{\prime}\right)
$$

Now $P_{3} P_{2}^{\prime \prime} / P_{2} P_{2}^{\prime}=P_{2} / 3^{\imath_{3}} P_{2}^{\prime}$ and $P_{3} / P_{3}^{\prime} \mid\left(P_{2} / 3^{\iota_{3}} P_{2}^{\prime}\right)^{2}$; hence,

$$
U=r+k P_{2} / 3^{\imath_{3}} P_{2}^{\prime}
$$

and

$$
U^{3} \equiv r^{3}+3 k r^{2} P_{2} / 3^{\iota_{3}} P_{2}^{\prime} \quad\left(\bmod 3^{\iota_{2}} P_{3} / P_{3}^{\prime}\right) .
$$

Also, by Lemma 6.1 and the definition of $\iota_{2}$, we have

$$
U^{3} \equiv-D \quad\left(\bmod 3{ }^{\imath}{ }_{2} P_{3} / P_{3}^{\prime}\right)
$$

thus,

$$
-D-r^{3} \equiv 3 k r^{2} P_{2} / 3^{\iota_{3}} P_{2}^{\prime} \quad\left(\bmod 3^{\iota_{2}} P_{3} / P_{3}^{\prime}\right)
$$

Since

$$
-D-r^{3} \equiv 0 \quad\left(\bmod P_{2} / P_{2}^{\prime}\right),
$$

we have

$$
\frac{-\left(D+r^{3}\right) 3^{\iota_{3}} P_{2}^{\prime}}{3^{\iota_{2}} P_{2}} \equiv 3^{1-\iota_{2}} k r^{2} \quad\left(\bmod P_{2} / 3^{\iota} P_{2}^{\prime} P_{2}^{\prime \prime}\right) .
$$

If $x$ is a solution of the congruence (possible by (6.1))

$$
3^{1-\imath_{2}} x r^{2} \equiv 1 \quad \bmod \left(P_{2} / 3^{\iota_{3}} P_{2}^{\prime} P_{2}^{\prime \prime}\right)
$$

then

$$
U \equiv r-x\left(D+r^{3}\right) / 3^{\iota_{2}} \quad\left(\bmod P_{3} / P_{3}^{\prime}\right)
$$

We must also have

$$
\left(P_{2}^{\prime \prime}\left(s+s^{\prime} \delta+\delta^{2}\right) / \sigma\right)^{2} \in a_{2}^{2}
$$

hence, there must exist $z_{1}, z_{2}, z_{3} \in$ (z) such that

$$
\begin{aligned}
P_{2}^{\prime \prime}\left(s^{\prime 2}+2 s\right) & =\sigma z_{3}, \\
P_{2}^{\prime \prime}\left(D+2 s s^{\prime}\right) & =\sigma z_{3} V^{\prime}+\sigma^{2} z_{2} P_{3}^{\prime}, \\
P_{2}^{\prime \prime}\left(s^{2}+2 D s^{\prime}\right) & =\sigma z_{3} V+\sigma^{2} z_{2} P_{3}^{\prime} U+\sigma^{2} z_{1} P_{3} .
\end{aligned}
$$

It follows that

$$
V^{\prime}\left(s^{\prime 2}+2 s\right) \equiv D+2 s s^{\prime} \quad\left(\bmod \sigma^{2} P_{3}^{\prime} / P_{2}^{\prime \prime}\right)
$$

and

(6.6) $V\left(s^{\prime 2}+2 s\right) \equiv s^{2}+2 D s^{\prime}-U\left(D+2 s s^{\prime}\right)+U V^{\prime}\left(s^{\prime 2}+2 s\right)\left(\bmod \sigma^{2} P_{3} / P_{2}^{\prime \prime}\right)$.

By Lemma 5.6, we may assume that $s$ and $s^{\prime}$ have been selected such that $\left(s^{\prime 2}+2 s, 3^{\iota_{0}+\iota_{1}} P_{2}\right)=3^{\iota_{1}}$; hence, we can solve

$$
y\left(s^{\prime 2}+2 s\right) / 3^{\iota_{1}} \equiv 1 \quad\left(\bmod 3^{\iota_{0}} P_{2}\right)
$$

for $y$. Since $D+2 s s^{\prime}=s^{\prime}\left(s^{\prime 2}+2 s\right)+D-s^{\prime 3}$, we have from (6.5)

$$
\left(V^{\prime}-s^{\prime}\right)\left(s^{\prime 2}+2 s\right) / 3^{\iota_{1}} \equiv\left(D-s^{\prime 3}\right) / 3^{\iota_{1}} \quad\left(\bmod 3^{\imath} P_{3}^{\prime}\right) \text {. }
$$


Now

$$
D-s^{\prime 3} \equiv 0 \quad\left(\bmod \sigma P_{2}^{\prime} / P_{2}^{\prime \prime}\right)
$$

thus,

$$
\frac{\left(V^{\prime}-s^{\prime}\right)}{P_{2}^{\prime}} \frac{\left(s^{\prime 2}+2 s\right)}{3^{\iota_{1}}} \equiv \frac{D-s^{\prime 3}}{3^{\iota_{1}} p_{2}^{\prime}} \quad\left(\bmod 3^{\iota^{+} \iota_{3}} P_{2}^{\prime}\right) .
$$

Since $3^{\imath_{3}} P_{2}^{\prime} \mid P_{2}$, it follows that

$$
V^{\prime} \equiv s^{\prime}+y\left(D-s^{\prime 2}\right) / 3^{\iota_{1}} \quad\left(\bmod 3^{\iota^{\prime \prime}} P_{3}^{\prime}\right)
$$

From (6.6), we get

$$
\begin{aligned}
\left(s^{\prime 2}+2 s\right) V \equiv & \left(U V^{\prime}+s-s^{\prime} U\right)\left(s^{\prime 2}+2 s\right) \\
& -\left(s^{2}-2 s^{\prime} D+s s^{\prime 2}+U\left(D-s^{\prime 3}\right)\right) \quad\left(\bmod \sigma^{2} P_{3} / P_{2}^{\prime \prime}\right) .
\end{aligned}
$$

Since $D-s^{\prime 3} \equiv 0\left(\bmod 3{ }^{\iota_{3}} \sigma P_{2}^{\prime} / P_{2}^{\prime \prime}\right), r \equiv U\left(\bmod P_{2} / 3^{\iota_{3}} P_{2}^{\prime}\right)$ and $3^{\iota_{1}} \mid \sigma / P_{2}^{\prime \prime}$, we see that

$$
U\left(D-s^{\prime 3}\right) \equiv r\left(D-s^{\prime 3}\right) \quad\left(\bmod 3^{\iota_{1}} P_{2}\right) .
$$

By Corollary 5.4.2, we have

$$
s^{2}-2 s^{\prime} D+r s^{\prime 2}+U\left(D-s^{\prime 3}\right) \equiv 0 \quad\left(\bmod 3^{\iota} P_{2}\right)
$$

hence,

$$
\left(\frac{s^{\prime 2}+2 s}{3^{\iota_{1}}}\right)\left(\frac{V-s+s^{\prime} U-U V^{\prime}}{P_{2}}\right) \equiv \frac{-\left(s^{2}-2 s^{\prime} D+s s^{\prime 2}+U\left(D-s^{\prime 3}\right)\right)}{3^{\iota_{1}} P_{2}}
$$

$\left(\bmod 3^{\imath-\iota_{3}} P_{2} / P_{2}^{\prime \prime}\right)$

Therefore

(6.9) $V \equiv s-s^{\prime} U+U V^{\prime}-y\left(s^{2}-2 s^{\prime} D+s s^{\prime 2}+U\left(D-s^{\prime 3}\right)\right) / 3^{\iota_{1}}\left(\bmod 3^{\iota_{1}} P_{3}\right)$.

Since we now have a basis for $\mathfrak{b}_{1}$ and one for $\mathfrak{b}_{2}$, we must next find a basis of $\mathfrak{b}_{1} \mathfrak{b}_{2}$. Let $\mathfrak{b}_{1} \mathfrak{b}_{2}$ have a basis $\left\{Q, Q^{\prime}(M+\delta), Q^{\prime \prime}\left(N+N^{\prime} \delta+\delta^{2}\right) / \sigma\right\}$. Since $\operatorname{gcd}\left(P_{3}, P_{1}\right)=1$, we have $Q=P_{3} P_{1}, Q^{\prime}=P_{3}^{\prime} P_{1} / P_{1}^{\prime} S, Q^{\prime \prime}=S / P_{1}^{\prime \prime}$,

$$
\begin{gathered}
M \equiv-D \quad\left(\bmod P_{1}^{\prime} S\right), \quad N^{\prime} \equiv D \quad\left(\bmod \sigma P_{1} / Q^{\prime \prime} P_{1}^{\prime} S\right) \\
M \equiv U \quad\left(\bmod P_{3} / P_{3}^{\prime}\right), \quad N^{\prime} \equiv V^{\prime} \quad\left(\bmod \sigma P_{3}^{\prime} / Q^{\prime \prime}\right) \\
N \equiv D^{2}-D\left(N^{\prime}-D\right) \quad\left(\bmod \sigma P_{1}^{\prime \prime} P_{1} / S\right) \\
N \equiv V+U\left(N^{\prime}-V^{\prime}\right) \quad\left(\bmod \sigma P_{1}^{\prime \prime} P_{3} / S\right)
\end{gathered}
$$

by Theorem 5.3. Since $3^{\iota_{0}} \mid \sigma / Q^{\prime \prime}$, we have

$$
N^{\prime} \equiv D \quad\left(\bmod \sigma P_{1} /\left(3^{\iota_{0}} Q^{\prime \prime} P_{1}^{\prime} S\right)\right) .
$$

Further

$$
N \equiv D^{2}-D\left(N^{\prime}-D\right) \quad\left(\bmod \sigma P_{1}^{\prime \prime} P_{1} / 3^{\iota_{0}} S\right)
$$

and

$$
\begin{aligned}
N & \equiv V+U\left(N^{\prime}-V^{\prime}\right) \\
& \equiv s-s^{\prime} U+U N^{\prime}-y\left(s^{2}-2 s^{\prime} D+2 s^{\prime 2}+U\left(D-s^{\prime 3}\right)\right) / 3^{\iota_{1}} \\
& \equiv s-s^{\prime} M+M N^{\prime}-y\left(s^{2}-2 s^{\prime} D+2 s^{\prime 2}+M\left(D-s^{\prime 3}\right)\right) / 3^{\iota_{1}} \quad\left(\bmod 3^{\iota_{0}} P_{3}\right)
\end{aligned}
$$


by (6.9) and (6.8). Since $\left(3^{\iota_{1}} P_{3}, \sigma P_{1}^{\prime \prime} P_{1} / 3^{\iota} S\right)=1$, we can solve for $N^{\prime}\left(\bmod \sigma Q^{\prime} / Q^{\prime \prime}\right)$ and $N\left(\bmod \sigma Q / Q^{\prime \prime}\right)$ by using the Chinese Remainder Theorem.

Now since $\Omega_{i} \sim R_{1}$, we know that a canonical basis of $R_{i}$ must have the form

$$
\left\{1,\left|e_{i}\right| \frac{m+\delta}{\sigma_{i}}, \frac{n+n^{\prime} \delta+\delta^{2}}{\sigma_{i}}\right\} .
$$

Also $\because R$, corresponds to an ideal a with basis $\left\{P, P^{\prime}(m+\delta), P^{\prime \prime}\left(n+n^{\prime} \delta+\delta^{2}\right) / \sigma\right\}$. Further,

$$
P^{\prime \prime}=\sigma / d, \quad P^{\prime}=P^{\prime \prime}\left|e_{i}\right| / \sigma, \quad P=P^{\prime \prime} \sigma_{i} / \sigma,
$$

where $d=\operatorname{gcd}\left(\sigma, e_{i}\right)$. Put $P_{1}=\operatorname{gcd}\left(\sigma_{i} / d, J\right), P_{1}^{\prime}=\operatorname{gcd}\left(e_{i} / d, J\right), P_{2}^{\prime \prime}=\operatorname{gcd}\left(3, P^{\prime \prime}\right)$, $P_{2}=P / P_{1}, P_{2}^{\prime}=P^{\prime} / P_{1}^{\prime}, P_{1}^{\prime \prime}=P^{\prime \prime} / P_{2}^{\prime \prime}, S=\operatorname{gcd}\left(\sigma, P_{1}\right)$,

$$
\begin{aligned}
& \iota_{0}=\left\{\begin{array}{ll}
1, & \iota=1 \text { and } 3 \mid P_{2}, \\
0 & \text { otherwise; }
\end{array} \iota_{1}= \begin{cases}1, & 3 \mid \sigma / P_{2}^{\prime \prime}, \\
0 & \text { otherwise; }\end{cases} \right. \\
& \iota_{2}= \begin{cases}1, & \iota_{0}=1, \text { and either } P_{2}^{\prime \prime}=3 \text { or } n^{\prime 2}+2 n \equiv 6(\bmod 9), \\
0 & \text { otherwise; }\end{cases} \\
& \iota_{3}= \begin{cases}1, & \iota_{0}=1, P_{2}^{\prime \prime} \neq 3,3 \mid P_{2} / P_{2}^{\prime}, n^{\prime 2}+2 n \equiv 3(\bmod 9), \\
0 & \text { otherwise. }\end{cases}
\end{aligned}
$$

If $\operatorname{gcd}\left(3^{\iota_{1}} P_{2},\left(n^{\prime 2}+2 n\right) / 3^{\iota_{1}}\right) \neq 1$, replace $n^{\prime}$ by $n^{\prime}+\sigma k P_{2}^{\prime} / P_{2}^{\prime \prime}$ and $n$ by $n+$ $k m \sigma P_{2}^{\prime} / P_{2}^{\prime \prime}$, where $k$ is selected such that this ged is 1 .

THEOREM 6.2. If ${ }^{R}$, , has a canonical basis $(6.10)$ such that

$$
\operatorname{gcd}\left(3^{\iota_{0}} P_{2},\left(n^{\prime 2}+2 n\right) / 3^{\iota_{1}}\right)=1 \text {, }
$$

then

$$
\mathrm{GR}_{i}^{2}=\left\langle 1,|f|(M+\delta) / \sigma,\left(N+N^{\prime} \delta+\delta^{2}\right) / \sigma\right\rangle,
$$

where $f=\sigma Q^{\prime} / Q^{\prime \prime}, \rho=\sigma Q / Q^{\prime \prime}, Q^{\prime \prime}=S / P_{1}^{\prime \prime}, Q^{\prime}=3^{\iota_{3}} P_{2}^{\prime 2} P_{1} / P_{1}^{\prime} S, Q=P_{2}^{2} P_{1} / 3^{\iota_{3}} P_{2}^{\prime \prime}$. Also, $M, N^{\prime}$ and $N$ can be determined from the congruences.

$$
\begin{aligned}
& M \equiv-D \quad\left(\bmod P_{1}^{\prime} S\right), \\
& M \equiv m-x\left(D+m^{3}\right) / 3^{\iota_{2}}\left(\bmod P_{2}^{2} / 3^{2 \iota_{3}} P_{2}^{\prime 2} P_{2}^{\prime \prime}\right) \text {, } \\
& N^{\prime} \equiv D \quad\left(\bmod \sigma P_{1} /\left(3^{\iota_{0}} Q^{\prime \prime} P_{1}^{\prime} S\right)\right) \text {, } \\
& N^{\prime} \equiv n^{\prime}+y\left(D-n^{\prime 3}\right) / 3^{\iota_{1}} \quad\left(\bmod 3^{\iota_{0}+\iota_{3}} P_{2}^{\prime 2}\right) \text {, } \\
& N \equiv 2 D^{2}-D N^{\prime} \quad\left(\bmod \sigma P_{1}^{\prime \prime} P_{1} / 3^{\iota_{0}} S\right) \text {, } \\
& N \equiv n-n^{\prime} M+M N^{\prime}-y\left(n^{2}-2 n^{\prime} D+2 n^{\prime 2}+M\left(D-n^{\prime 3}\right)\right) / 3^{\iota_{1}} \\
& \left(\bmod 3^{\iota_{0}-\iota_{3}} P_{2}^{2} / P_{2}^{\prime \prime}\right) \text {, }
\end{aligned}
$$

where

$$
3^{1-\iota_{2}} x m^{2} \equiv 1 \quad\left(\bmod P_{2} / 3^{\iota_{3}} P_{2}^{\prime \prime} P_{2}^{\prime}\right)
$$

and

$$
y\left(n^{\prime 2}+2 n\right) / 3^{\iota_{1}} \equiv 1 \quad\left(\bmod 3^{\iota_{0}} P_{2}\right) .
$$




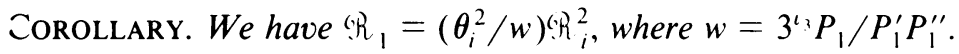

Proof. Follows from the above results and Lemma 2.1.

We also point out here that

$$
\sigma_{i}^{2}=\left(3^{\iota_{3}} \sigma P_{1} S / P^{\prime \prime} P_{1}^{\prime \prime 2}\right) \rho
$$

and

$$
\left(\sigma_{i}^{2} / \sigma e_{i}\right)^{2}=\left(P_{1} 3^{\iota_{3}} / P_{1}^{\prime} P_{1}^{\prime \prime}\right)^{3} \rho^{2} / \sigma|f|
$$

Hence

$$
\rho \leqslant \sigma_{i}^{2}<9 D^{2}, \quad \rho^{2} /|f|<.9 D^{2}
$$

by (6.1) and (6.2) of [13]. Also, if $s_{1}=\mathrm{sh}_{i}^{2}$ in Section 4 and $\gamma_{r} \bar{s}_{r}=\bar{s}_{1}$, where $\Gamma_{r}$ $\left(\approx \gamma_{r}\right) \in \mathcal{E}_{1}$, then $N\left(\gamma_{r}\right)<1$ and

$$
\rho_{r}^{2} /\left|f_{r}\right|<\rho^{2} /|f|<9 D^{2}
$$

from (4.5).

7. Reduction. Let $\stackrel{R}{R}$ be a lattice with basis a basis of $=[\delta]$. In this section and the two following sections, we will show how to find a reduced lattice 5 such that $s \sim$ ( $R$. In the process of doing this, we will also show how to find $\gamma \in \because(\delta)$ such that $\gamma \varsigma=\ell_{R}$. In order to do this, we will make use of much of the reasoning employed in [18] and several of the results provided in that work. We begin with several simple lemmas; but we must first describe what is meant by the puncture of a point $\Omega(\approx \omega) \in G$. We define the puncture of $\Omega$ as in [6] and [18]; that is, we say that it is that point $\omega_{p}=\left(\xi_{\omega}, \eta_{\omega}\right)$ in the $x-y$ plane such that

$$
\xi_{\omega}=\left(2 \omega-\omega^{\prime}-\omega^{\prime \prime}\right) / 2, \quad \eta_{\omega}=\left(\omega^{\prime}-\omega^{\prime \prime}\right) / 2 i .
$$

If we put $\zeta_{\omega}=\left(\omega^{\prime}+\omega^{\prime \prime}\right) / 2$, we have $\omega=\xi_{\omega}+\zeta_{\omega}$. If

$$
\delta^{3}=D \quad \text { and } \quad \omega=\left(q_{1}+q_{2} \delta+q_{3} \delta^{2}\right) / \rho, \quad\left(q_{1}, q_{2}, q_{3}, \rho \in \stackrel{\Xi}{*}\right)
$$

then

$$
\begin{gathered}
\xi_{\omega}=3 \delta\left(q_{2}+q_{3} \delta\right) / 2 \rho, \quad \eta_{\omega}=\sqrt{3} \delta\left(q_{2}-q_{3} \delta\right) / 2 \rho . \\
\zeta_{\omega}=\left(2 q_{1}-\delta q_{2}-\delta^{2} q_{3}\right) / 2 \rho .
\end{gathered}
$$

We note that there exists an infinitude of points of $R$ which have the same puncture $\omega_{p}$ as $\Omega$ does. Let $\Omega^{*}\left(\approx \omega^{*}\right)$ be that one of this infinitude of points such that $\Omega^{*} \in \mathcal{E}$ and $\left|\omega^{*}\right|$ is minimal. Now $\Omega^{*}$ does not necessarily exist, but, it certainly exists if $\left|\eta_{\omega}\right|<\sqrt{3} / 2$. In the following lemmas we will assume that $\Omega(\approx \omega)$ is a point of $R$ such that $\omega \notin 2$.

Lemma 7.1. Suppose $\Omega \in \mathcal{C}$ and $\omega<1$. If $\Omega$ has puncture $\left(\xi_{\omega}, \eta_{\omega}\right)$ with $\xi_{\omega}>0$, then $\Omega \in e_{1}$.

Proof. If $\omega>-1$, then clearly $\Omega \in \mathcal{C}_{1}$. If $\omega<-1$, then, since $\omega=\xi_{\omega}+\zeta_{\omega}$ and $\xi_{\omega}>0$, we must have $\zeta_{\omega}<-1$; by (2.2), this contradicts the fact that $\Omega \in \circlearrowright$.

LEMma 7.2. If $\Omega \in \mathcal{C}_{1}$ and $\Omega$ has puncture $\left(\xi_{\omega}, \eta_{\omega}\right)$, then $\xi_{\omega}<1+\sqrt{1-\eta_{\omega}^{2}}$. 
Proof. Follows easily from the inequalities $\omega=\xi_{\omega}+\zeta_{\omega}<1$ and $\left|\omega^{\prime}\right|^{2}=\zeta_{\omega}^{2}+\eta_{\omega}^{2}$ $<1$.

LEMMA 7.3. If $\Omega^{*}$ exists, $\Omega^{*} \notin \mathfrak{L}_{1},\left(\xi_{\omega}, \eta_{\omega}\right)$ is the puncture of $\Omega^{*}$ and $\xi_{\omega}>0$, then

$$
-1 \leqslant \zeta_{\omega}^{*}<1-\sqrt{1-\eta_{\omega}^{2}} \text {. }
$$

Proof. Since $\Omega^{*} \in \Theta^{\prime}$ and $\Omega^{*} \notin 心$, we must have $\omega^{*}>1$ by Lemma 7.1. Also, $\left|\zeta_{\omega}^{*}\right|<1$. If $\zeta_{\omega}^{*} \geqslant 1-\sqrt{1-\eta_{\omega}^{2}}$, then $\left(\zeta_{\omega}^{*}-1\right)^{2}+\eta_{\omega}^{2} \leqslant 1$ and $\Omega^{*}-(1,0,1) \in$ : Since $0<\omega^{*}-1<\omega^{*}$, this contradicts the definition of $\Omega^{*}$. The lemma follows.

LEMma 7.4. If $\Omega$ has puncture $\left(\xi_{\omega}, \eta_{\omega}\right), \Omega^{*}$ exists and $\left|\xi_{\omega}\right|<\sqrt{1-\eta_{\omega}^{2}}$, then $\Omega^{*} \in e_{1}$.

Proof. Since $(-\Omega)^{*}=-\Omega^{*}$, we see that if $\xi_{\omega}<0$, we can replace $\Omega$ by $-\Omega$ and $\xi_{\omega}$ by $-\xi_{\omega}>0$. Thus, we may assume $\xi_{\omega}>0$. If $\Omega^{*} \notin \mathcal{E}_{1}$, by Lemma 7.3 , we have $\zeta_{\omega^{*}}<1-\sqrt{1-\eta_{\omega}^{2}}$; hence, $\omega^{*}=\zeta_{\omega^{*}}+\xi_{\omega}<1$ and $\Omega^{*} \in 心$, by Lemma 7.1. Since this is a contradiction, the lemma follows.

LEMMA 7.5. Let $\Omega$ have puncture $\left(\xi_{\omega}, \eta_{\omega}\right)$ such that $\xi_{\omega}>0$ and $\left|\eta_{\omega}\right|<\sqrt{3} / 2$. Let

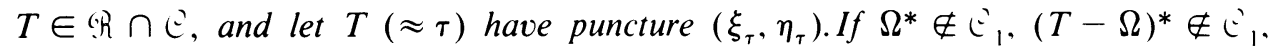
$\xi_{\tau}>\xi_{\omega}$ and $\eta_{\tau} \eta_{\omega}>0$, then $\tau>\omega^{*}$.

Proof. Follows by using Lemma 7.3 and the reasoning of Lemma 4.3 of [18].

LEMMA 7.6. Let $\Omega$ have puncture $\left(\xi_{\omega}, \eta_{\omega}\right)$ with $\xi_{\omega}>0$. If $T \in \Theta_{1} \cap$ ? h has puncture

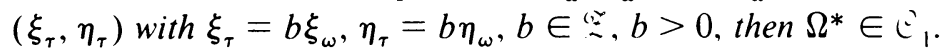

Proof. The result is certainly true for $b=1$. Suppose it is true for $b=k$, and suppose $\xi_{\tau}=(k+1) \xi_{\omega}, \eta_{\tau}=(k+1) \eta_{\omega}$. If $(T-\Omega)^{*} \in \mathbb{E}_{1}$, then since the puncture of $(T-\Omega)^{*}$ is $\left(k \xi_{\omega}, k \eta_{\omega}\right)$, the result is true by the induction hypothesis. If $(T-\Omega)^{*} \notin e_{1}$ and $\Omega^{*} \notin e_{1}$, then since $\xi_{\tau}>\xi_{\omega}>0, \eta_{\tau} \eta_{\omega}>0, \eta_{\omega}<\eta_{\tau} /(k+1)$ $<\sqrt{3} / 2$, we must have $\tau>\omega^{*}$ by Lemma 7.5. Since $\tau<1$, we have $\omega^{*}<1$, which, in view of Lemma 7.1, contradicts the assumption that $\Omega^{*} \notin \mathcal{E}_{1}$. The lemma is true by induction.

Let

$$
E=\left\{\omega_{p} \mid \Omega \in R\right\} .
$$

It is known (see [18,pp. 581-582]) that there exist $\phi_{p}, \psi_{p} \in E$ such that

$$
\mathcal{L}=\left\{a \phi_{p}+b \psi_{p} \mid a, b \in \stackrel{\stackrel{2}{z}}{=}\right.
$$

and

$$
\left\{\begin{array}{l}
\xi_{\phi}>\xi_{\psi}>0, \quad\left|\eta_{\phi}\right|<\left|\eta_{\psi}\right|, \quad \eta_{\phi} \eta_{\psi}<0, \\
\left|\eta_{\phi}\right|<1-\sqrt{3} / 4, \quad\left|\eta_{\psi}\right|>\sqrt{3} / 4, \quad\left|\eta_{\psi}\right|>\left(1-\left|\eta_{\phi}\right|\right) / 2,
\end{array}\right.
$$

where $\phi_{p}=\left(\xi_{\phi}, \eta_{\phi}\right)$ and $\psi_{p}=\left(\xi_{\psi}, \eta_{\psi}\right)$. Further, if $\{1, \mu, \nu\}$ is a basis of $\Re$, then

$$
\left(\begin{array}{c}
\mu_{p} \\
\nu_{p}
\end{array}\right)=H\left(\begin{array}{c}
\psi_{p} \\
\phi_{p}
\end{array}\right)
$$


whrre $H \in G L_{2}(\stackrel{\circlearrowleft}{*})$. Thus, if we transform the basis $\{1, \mu, \nu\}$ by $H^{T}$, we get a new basis $\{1, \psi, \phi\}$ of $\left(R\right.$ such that $\psi_{p}$ and $\phi_{p}$ are the punctures of $\Psi(\approx \psi)$ and $\Phi(\approx \phi)$, respectively.

We now prove the following important theorem.

THEOREM 7.7. If there exists $\Gamma(\approx \gamma) \in \mathcal{Q} R$ such that $\gamma \notin \mathcal{Z}$ and $\Gamma \in \mathcal{E}_{1}$, there must exist $\Theta(\approx \theta) \in\left(R\right.$ such that $\theta \notin \stackrel{\theta}{*}, \Theta \in \mathcal{E}_{1}$ and $\Theta$ has as its puncture one of: $\phi_{p}, \psi_{p}, \phi_{p}-\psi_{p}, \phi_{p}+\psi_{p}$, or $2 \phi_{p}+\psi_{p}$.

Proof. Since $\Gamma \in R, \Gamma$ has a puncture $\gamma_{p} \in \mathcal{R}$. Thus, $\gamma_{p}=a \phi_{p}+b \psi_{p}$ for some $a, b \in \stackrel{\Xi}{ }$. If $\xi_{\gamma}<0$, we can replace $\Gamma$ by $-\Gamma$; thus, we may assume, with no loss of generality, that $\xi_{\gamma}>0$.

If $a<0$, then $b>0$ and

$$
\left|\eta_{\gamma}\right|=|a|\left|\eta_{\phi}\right|+b\left|\eta_{\psi}\right|>1
$$

for $b \geqslant 2$. If $b=1$, then $\xi_{\gamma}<0$, which is a contradiction; hence, we must have $a \geqslant 0$. If $a=0$, we have $b>0$ and $\gamma_{p}=b \psi_{p}$. From Lemma 7.6 we see that $\psi_{p}$ is the puncture of $\Psi^{*}$, where $\Psi^{*} \in \mathcal{E}_{1} \cap \boldsymbol{Q}_{\mathrm{R}}$.

Suppose $a>0$ and $b \leqslant 0$. We have

$$
\left|\eta_{\gamma}\right|=a\left|\eta_{\phi}\right|+|b|\left|\eta_{\psi}\right|>1
$$

when $|b| \geqslant 2$. If $b=0$, we have $\gamma_{p}=a \phi_{p}$, and once again we see from Lemma 7.6 that $\phi_{p}$ is the puncture of $\Phi^{*}$, where $\Phi^{*} \in \mathcal{E}_{1} \cap$ 桹. If $b=-1$ and $a \geqslant 3$, then $\xi_{\gamma}>2 \xi_{\phi}$ and, since $\left|\eta_{\gamma}\right|<1$ and $\left|\eta_{\psi}\right|>\sqrt{3} / 4$, we get $\left|\eta_{\phi}\right|<(1-\sqrt{3} / 4) / 3$. Thus, $\sqrt{1-\eta_{\phi}^{2}}>.98$ and, if $\Phi^{*} \notin \mathfrak{C}_{1}$, we must have $2 \xi_{\phi}>1.96$ by Lemma 7.4. Now $\left|\eta_{\gamma}\right|>\left|\eta_{\psi}\right|>\sqrt{3} / 4$; hence, since $\Gamma \in \mathcal{E}_{1}$, we must, by Lemma 7.2 , have

$$
\xi_{\gamma}<1+\sqrt{1-\eta_{\gamma}^{2}}<1+\sqrt{13} / 4<1.96
$$

Since this is a contradiction, we see that if $b=-1$ and $a \geqslant 3$, then $\Phi^{*} \in \mathcal{C}_{1}$. If $b=-1$ and $a=2$, then $\gamma_{p}-\phi_{p}=\phi_{p}-\psi_{p}$. Also, $\eta_{\gamma}$ and $\eta_{\phi}$ have the same sign; thus, if neither $\Phi^{*}$ nor $(\Phi-\Psi)^{*}$ is in $e_{1}$, then by Lemma 7.5 , we must have $\gamma>\phi^{*}>1$. This contradicts the fact that $\Gamma \in \mathcal{e}_{1}$; hence, if $b=-1$ and $a=2$, then one of $\Phi^{*}$ or $(\Phi-\Psi)^{*}$ is in $\bigcup_{1}$. Also, if $a=1$ and $b=-1$, then $(\Phi-\Psi)^{*} \in \bigodot_{1}$.

Now suppose that $b \geqslant a>0$. If $d=b-a \geqslant 0$, we have

$$
\left|\eta_{\gamma}\right|=a\left|\eta_{\phi}+\eta_{\psi}\right|+d\left|\eta_{\psi}\right| \text {. }
$$

If $d=0$, then $(\Phi+\Psi)^{*} \in \mathcal{C}_{1}$ by Lemma 7.6. Assume that $d>0$. If $\left|\eta_{\phi}+\eta_{\psi}\right|>1 / 2$, then $\left|\eta_{\psi}\right|>1 / 2$ and $a+d<2$. Since $d>0$, this is impossible. If $\left|\eta_{\phi}+\eta_{\psi}\right|<1 / 2$, then $\left|\eta_{\phi+\psi}\right|=\left|\eta_{\phi}+\eta_{\psi}\right|<\sqrt{3} / 2$ and $(\Phi+\Psi)^{*}$ exists. If $(\Phi+\Psi)^{*} \in \bigodot_{1}$, we have finished the proof for this case. Suppose that $(\Phi+\Psi)^{*} \notin \bigodot_{1}$. Now $\eta_{\gamma}$ and $\eta_{\phi+\psi}$ have the same sign and $\xi_{\gamma}>\xi_{\phi+\psi}$; hence, from Lemma 7.5 it follows that if no point of $\mathscr{R} \cap \bigodot_{1}$ with puncture $(a-1)\left(\phi_{p}+\psi_{p}\right)+d \psi_{p}$ is in $\bigodot_{1}$, then $\gamma>(\phi+\psi)^{*}>1$, a contradiction. Thus, we must assume that there exists a point of $R$ with puncture $(a-1)\left(\phi_{p}+\psi_{p}\right)+d \psi_{p}$. By continuing this argument we must conclude that, since $a$ is a finite integer, there must be a point of $R$ in $\bigodot_{1}$ with puncture $d \psi_{p}$. By Lemma 7.6, we see that $\Psi^{*} \in \bigodot_{1}$. 
It remains to consider the case of $a>b \geqslant 1$. If $a \geqslant 3$, we have $\xi_{\gamma}>3 \xi_{\phi}$. If $\Phi^{*} \notin \mathrm{E}_{1}$, then $\xi_{\phi}>\sqrt{1-\eta_{\phi}^{2}}>$.8. Also, $\xi_{\gamma}<1+\sqrt{1-\eta_{\gamma}^{2}}<2$. Since $\xi_{\gamma}>3(.8)$, we have a contradiction; thus, if $a>b \geqslant 1$, we can only have $a=2, b=1$.

Let $\alpha^{\prime}$ ' be a set of points $(a, b) \in{ }^{\prime 2}$ such that if $(h$ is not reduced, then one of $(a \Phi+b \Psi)^{*} \in \mathcal{E}_{1}$. By Theorem 7.6, we see that we can have $\hat{A} \subseteq\{(1,0),(1,0)$, $(1,-1),(1,1),(2,1)\}$. In certain cases $c^{\prime}$ is actually a proper subset of this set.

Corollary 7.7.1. If $\left|\eta_{\psi}\right|<\sqrt{3} / 2$, then $\|^{\prime}=\{(1,0),(0,1),(1,-1)\}$.

Proof. When $\left|\eta_{\psi}\right|<\sqrt{3} / 2$, we know that $\Psi^{*}$ exists. Since $\Phi^{*}$ also exists, $\eta_{\phi} \eta_{\psi}<0$ and $\xi_{\gamma}>\xi_{\psi}, \xi_{\phi}$ when $\gamma_{p}=\phi_{p}+\psi_{p}$ or $\gamma_{p}=2 \phi_{p}+\psi_{p}$, we see that $\gamma$ cannot be less

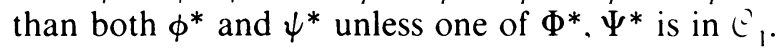

COROllary 7.7.2. If $\left|\eta_{\psi}\right|<1.19$, then $\hat{~}^{\prime}=\{(1,0),(0,1),(1,-1),(1,1)\}$.

Proof. Suppose that $\Gamma \in \mathcal{E}_{\text {, }}$ and $\gamma_{p}=2 \phi_{p}+\psi_{p}$. If $\eta_{\gamma}$ and $\eta_{\phi}$ have the same sign, then since $\xi_{\gamma}>\xi_{\phi}$, we must have $\Phi^{*}$ or $(\Phi+\Psi)^{*}$ lying in $\mathcal{E}_{1}$, or $\gamma>\phi^{*}$ by Lemma 7.5. If $\Phi^{*} \notin \mathfrak{E}_{1}$, then $\gamma>\phi^{*}>1$ and $\Gamma \notin \mathfrak{E}_{1}$.

If $\eta_{\gamma}$ and $\eta_{\phi}$ do not have the same sign, then $\eta_{\gamma}$ and $\eta_{\phi+\psi}$ must have the same sign. If $\left|\eta_{\phi+\psi}\right|<\sqrt{3} / 2$, then, again by Lemma 7.5, we must have $\Phi^{*}$ or $(\Phi+\Psi)^{*} \in \mathcal{C}_{1}$ or $\gamma>(\phi+\psi)^{*}$. If $\Phi^{*},(\Phi+\Psi)^{*} \notin \mathcal{E}_{1}$, then $\gamma>(\phi+\psi)^{*}>1$ and $\Gamma \notin \mathcal{C}_{1}$. Thus, we will assume that $\left|\eta_{\phi}+\eta_{\psi}\right|=\left|\eta_{\psi}\right|-\left|\eta_{\phi}\right|>\sqrt{3} / 2$. We have $\left|\eta_{\gamma}\right|=\left|\eta_{\psi}\right|-2\left|\eta_{\phi}\right|>\sqrt{3} / 2$ $-\left|\eta_{\phi}\right|$. If $\Phi^{*} \notin \mathcal{E}_{1}$, we get $\xi_{\phi}>\sqrt{1-\eta_{\phi}^{2}}$ from Lemma 7.4. Also, since $\Gamma \in \bigodot_{1}$, we have $\xi_{\gamma}<1+\sqrt{1-\eta_{\gamma}^{2}}$ from Lemma 7.2. Now since $\left|\eta_{\psi}\right|>\sqrt{3} / 2+\left|\eta_{\phi}\right|$ and $\left|\eta_{\psi}\right|<$ 1.19 , we find that $\left|\eta_{\phi}\right|<1 / 3$. Further,

$$
f(x)=2 \sqrt{1-x^{2}}-\sqrt{1-(\sqrt{3} / 2-x)^{2}}
$$

is a strictly decreasing function for $0<x<1$ and $f(1 / 3)>1.039$; hence,

$$
\xi_{\gamma}>2 \xi_{\phi}>2 \sqrt{1-\eta_{\phi}^{2}}>1+\sqrt{1-\left(\sqrt{3} / 2-\left|\eta_{\phi}\right|\right)^{2}}>1+\sqrt{1-\eta_{\gamma}^{2}} .
$$

Since this is a contradiction, we see that we can eliminate $(2,1)$ from the set given above.

With these results we are now in a position to develop an algorithm for finding a reduced lattice $\delta$ and $\gamma \in \mathscr{K}$ such that $\mathscr{R}=\gamma \delta$. Let $\gamma_{1}=1, \mathcal{S}_{1}=\Re$. We first find

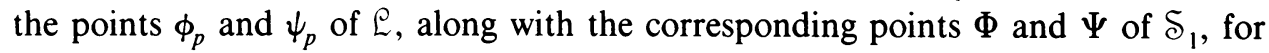
which $\phi_{p}$ and $\psi_{p}$ are the respective punctures. We can then find the points $a \Phi+b \Psi$ of $\mathcal{S}_{1}$ with $(a, b) \in \mathbb{Q}$ and then determine $(a \Phi+b \Psi)^{*}$. If none of these points is in $\bigodot_{1}$, we know that $\delta_{1}$ is already reduced and $\gamma=\gamma_{1}$. If one of them is in $\bigodot_{1}$, let $\Gamma_{g}^{(1)}$ $\left(\approx \gamma_{g}^{(1)}\right)=(a \Phi+b \Psi)^{*}$ be that point such that $\Gamma_{g}^{(1)} \in \mathcal{C}_{1}$ and $\left|\gamma_{g}^{(1)}\right|$ is least. Put $\gamma_{g}^{(1)}=a \phi+b \psi$, and $\gamma_{h}^{(1)}=\psi$, when $(a, b) \neq(0,1)$ or $\gamma_{h}^{(1)}=\phi$ when $(a, b)=(0,1)$. Then $\left\{1, \gamma_{g}^{(1)}, \gamma_{h}^{(1)}\right\}$ is a basis of $\delta_{1}$ and, if we define $\delta_{2}=\left|1 / \gamma_{g}^{(1)}\right| \delta_{1}$, we have $\delta_{2} \sim \mathcal{S}_{1}$. We then repeat the algorithm on $\delta_{2}$ to find that either $\delta_{2}$ is reduced or $\Gamma_{g}^{(2)}$ $\left(\approx \gamma_{g}^{(2)}\right) \in \delta_{2} \cap \mathcal{C}$. We repeat the above procedure to obtain $\delta_{3}=\left|1 / \gamma_{g}^{(2)}\right| \delta_{2}$ and then continue the entire process until we ultimately find $\delta_{k}=\left|1 / \gamma_{g}^{(k-1)}\right| \delta_{k-1}$ such 
that $s_{k}$ is reduced. That this must eventually occur follows from the fact that $\hat{s}_{j}=\left(1 / \gamma_{j}\right) \tilde{s}_{1}$, where

$$
\gamma_{i}=\prod_{i=1}^{i}\left|\gamma_{g}^{(i)}\right|
$$

hence $\left|\gamma_{j}\right|<1$ and $\left|\gamma_{j}^{\prime}\right|<1$. Since there can only be a finite number of points in $\bar{s}_{1} \cap \mathcal{E}_{1}$, the algorithm must terminate and $\gamma=\gamma_{k}$. It can also be shown that the value of $k$ here is $O(\log |\Delta|)$.

Thus, in order to find $s$ and $\gamma$, we must know how to find $\psi_{p}, \phi_{p}, \Psi, \Phi$, $(a \Phi+b \Psi)^{*}$ for $(a, b) \in t^{\prime}$ and be able to determine whether or not $(a \Phi+b \Psi)^{*} \in$ $c_{1}$. We will show how to solve these problems when $\delta^{3}=D$ in the next two sections.

8. The Algorithm for Determining $\phi$ and $\psi$ when $\delta^{3}=D$ and $D>10^{5}$. The problem of this section and the next is really that of developing an algorithm which determines whether or not there exists a $\gamma_{g}(>0)$ such that $\Gamma_{g}\left(\approx \gamma_{g}\right) \in R \cap \mathcal{E}_{1}$ and, if such a $\gamma_{g}$ does exist, to find it. Because of the precision problems mentioned in [18], we will produce an algorithm which will require that we perform our operations on integers only.

Let $(h$ have a canonical basis

$$
\left\{1,|f|(m+\delta) / \rho,\left(n+n^{\prime} \delta+\delta^{2}\right) / \rho\right\} .
$$

We may certainly assume that $|m|<\rho /|f|,|n|<\rho,\left|n^{\prime}\right|<|f|$. If*** $|f| \leqslant[\delta]$, transform this basis by $K=\left(\begin{array}{ll}1 & 0 \\ 0 & 1\end{array}\right)$; if $|f|>[\delta]$ and $n^{\prime}=0$, transform this basis by $K=\left(\begin{array}{ll}0 & 1 \\ 1 & 0\end{array}\right)$.

If $|f|>[\delta]$ and $n^{\prime} \neq 0$, let

$$
n^{\prime} /|f|=\left\langle q_{0}, q_{1}, q_{2}, \ldots, q_{k}\right\rangle
$$

be the continued fraction expansion of $n^{\prime} /|f|$, and let $A_{-2}=B_{-1}=0, A_{-1}=B_{-2}=1$, $A_{r+1}=q_{r+1} A_{r}+A_{r-1}, B_{r+1}=q_{r+1} B_{r}+B_{r-1}$. Then $A_{m} / B_{m}=\left\langle q_{0}, q_{1}, q_{2}, \ldots, q_{m}\right\rangle$. Let $d=\operatorname{gcd}\left(n^{\prime}, f\right)$. Since $d \mid D$; we see that $D f \mid d^{2}$ is an integer. If $D|f| / d^{2} \leqslant\left[\delta^{2}\right]$, put $j=k$; then $B_{j}=|f| / d<\sqrt{|f| / \delta}$. If $D|f| / d^{2}>\left[\delta^{2}\right]$, find $j$ such that

$$
D B_{j}^{2} \leqslant\left[\delta^{2}\right]|f| \text { and } D B_{j+1}^{2}>\left[\delta^{2}\right]|f| \text {. }
$$

Since $D f / d^{2}$ is an integer, we know that $B_{k}=|f| / d>\sqrt{|f| / \delta}$, and therefore such a $j$ must exist. Further,

$$
B_{j}<\sqrt{|f| / \delta}
$$

Put

$$
K=\left(\begin{array}{cc}
A_{j} & A_{j-1} \\
-B_{j} & -B_{j-1}
\end{array}\right) .
$$

We have $|K|= \pm 1$. Transform the canonical basis by $K$. If the new basis of $\mathscr{R}$ has the form

$$
\left\{1, \frac{m_{1}+m_{2} \delta+m_{3} \delta^{2}}{\rho}, \frac{n_{1}+n_{2} \delta+n_{3} \delta^{2}}{\rho}\right\}
$$

***We use the notation $[\alpha]$ to denote that integer which satisfies $\alpha-1<[\alpha] \leqslant \alpha$. 
then $m_{3}=-B_{j} ; \quad n_{3}=-B_{j-1}, \quad m_{2}=A_{1}|f|-B_{j} n^{\prime}, \quad n_{2}=A_{j-1}|f|-B_{j-1} n^{\prime} ; \quad$ thus $\left|n_{3}\right|<\left|m_{3}\right|<\sqrt{|f| / \delta}$. Also, if $D|f| / d^{2} \leqslant\left[\delta^{2}\right]$, then $m_{2}=0$. Now it is well known that

$$
\left|\frac{n^{\prime}}{|f|}-\frac{A_{1}}{B_{1}}\right|<\frac{1}{B_{j} B_{j+1}} ;
$$

hence, if $D|f| / d^{2}>\left[\delta^{2}\right]$, we find that

$$
\left|m_{2}\right|<|f| / B_{j+1}<\sqrt{\delta|f|}\left(\sqrt{\delta^{2} /\left[\delta^{2}\right]}\right)<1.0003 \sqrt{\delta|f|} \text { for } D>10^{5} .
$$

Also, since $n_{2}$ and $m_{2}$ have different signs, $m_{3} n_{3}>0$, and $\left|m_{2} n_{3}-m_{3} n_{2}\right|=|f|$, we find that $\left|n_{2}\right|<\left|f / m_{3}\right|$.

Thus, after transforming our normal basis by whichever of the matrices $K$ we select, we obtain a new basis of the form (8.1), where

$$
\begin{gathered}
0<m_{1}, n_{2}<\rho, \quad\left|n_{2}\right| \leqslant|f|, \quad\left|m_{2}\right|<1.0003 \sqrt{\delta|f|}, \\
\delta\left|n_{3}\right|<\sqrt{\delta|f|}, \quad \delta\left|m_{3}\right|<\sqrt{\delta|f|} .
\end{gathered}
$$

We call this step where we find this new basis (8.1) the pre-reduction step.

Incidentally, if $\mu=(m+\delta)|f| / \rho$, we see that $\xi_{\mu}=3 \delta|f| / 2 \rho, \eta_{\mu}=\sqrt{3} \delta|f| / 2 \rho$, $\xi_{\mu}^{2}+\eta_{\mu}^{2}=3 f^{2} \delta^{2} / \rho^{2}$. Thus, if $\left(R\right.$ is a reduced lattice, like $Q_{R}$, then, by Lemma 7.4, $\rho /|f|<\sqrt{3} \delta$. This allows us to improve the result (6.3) of [18] to

$$
\sigma_{i} /\left|e_{i}\right|<\sqrt{3} \delta
$$

Let $M \approx \mu=\left(m_{1}+m_{2} \delta+m_{3} \delta^{2}\right) / \rho, \nu=\left(n_{1}+n_{2} \delta+n_{3} \delta^{2}\right) / \rho$, where (8.2) is true. We have $\left|m_{2}\right|+\left|m_{3} \delta\right|<3 \sqrt{\delta|f|}$. Now, if $\rho^{2} /|f|>9.0003 D$, we have $3 \sqrt{\delta|f|}<$ $\rho / \delta$ and $\left|\eta_{\mu}\right|=\sqrt{3} \delta\left|m_{2}-m_{3} \delta\right| / 2 \rho<\sqrt{3} / 2$; hence, $M^{*}$ exists. We also have

$$
3\left(m_{2}+m_{3} \delta\right)^{2}+\left(m_{2}-m_{3} \delta\right)^{2}=4\left(m_{2}^{2}+m_{2} m_{3} \delta+m_{3}^{2} \delta^{2}\right)<4 \rho^{2} / 3 \delta^{2} .
$$

Hence $\eta_{\mu}^{2}+\xi_{\mu}^{2}<1$. By Lemma 7.4, we see that $M^{*} \in e_{1}$.

We now know that if $\rho^{2} /|f|>9.0003 D$, we can easily, by the pre-reduction process described above, find a basis $\{1, \mu, \nu\}$ of $\Re$ such that $M^{*}\left(\approx \mu^{*}\right) \in \mathcal{C}_{1}$ and $\mu^{*}>0$. We now show, given $\mu$ and the fact that $\left|m_{1}\right|<\rho$, how to find $\lambda$ such that 政 $=\langle 1, \lambda, \nu\rangle, \Lambda(\approx \lambda) \in e_{1}$ and $\lambda>0$.

Let $\lambda=\left(m_{1}^{*}+m_{2} \delta+m_{3} \delta^{2}\right) / \rho$. We first note that if $M^{*} \in \mathcal{C}_{1}$, then $|\lambda|<1$, $\left|\lambda^{\prime}\right|=\left|\lambda^{\prime \prime}\right|<1$. Since $3 m_{i}^{*} / \rho=\lambda+\lambda^{\prime}+\lambda^{\prime \prime}$, we have $\left|m_{i}^{*}\right|<\rho$. If $m_{1}<0$, replace $m_{1}$ by $m_{1}+\rho$. We now have $0<m_{1}<\rho$. Since $m_{1}^{*} \equiv m_{1}(\bmod \rho)$ and $\left|m_{1}^{*}\right|<\rho$, we see that $m_{1}^{*}$ is either $m_{1}$ or $m_{1}-\rho$. Put $m^{*}$ equal to that one of $m_{1}$ or $m_{1}-\rho$ which has least absolute value.

Put

$$
\begin{gathered}
\bar{m}_{1}=\left(m_{1}^{* 2}-D m_{2} m_{3}\right) /|f|, \quad \bar{m}_{2}=\left(D m_{3}^{2}-m_{1}^{*} m_{2}\right) /|f|, \\
\bar{m}_{3}=\left(m_{2}^{2}-m_{1}^{*} m_{3}\right) /|f|, \quad \tau=\rho /|f|, \quad Q=\rho \tau, \\
\rho \Sigma=m_{1}^{*} \bar{m}_{1}+D\left(m_{2} \bar{m}_{3}+m_{3} \bar{m}_{2}\right) .
\end{gathered}
$$

By using the reasoning of Section 9 of [18], we see that in order for $\Lambda \in \mathcal{C}$, we must have

$$
\Sigma^{2}+3 \tau\left(\bar{m}_{1} \rho-m_{1}^{*} \Sigma\right)<Q^{2} .
$$


Also, if $|\lambda|<1$, we must have $N(\lambda-1)<0$ and $N(\lambda+1)>0$; hence,

$$
-\left(Q+3 \bar{m}_{1}\right)<\Sigma+3 \tau m_{1}^{*}<Q+3 \bar{m}_{1} \text {. }
$$

Thus, if (8.4) and (8.5) hold, we have our value of $\lambda$. If one of them does not hold, then we can put $m_{1}^{*}$ equal to the other of the two $m_{1}, m_{1}-\rho$, from which we made our first selection. Since we selected the value of $m_{1}^{*}$ such that $\left|m_{1}^{*}\right|$ is minimal, we usually find that the first selected value of $m_{1}^{*}$ works. If $\Sigma<0$, replace $m_{1}^{*}, m_{2}, m_{3}, \Sigma$ by $-m_{1}^{*},-m_{2},-m_{3}$, and $-\Sigma$. We now have $\lambda>0$. We call this process of finding $\lambda$ from $\mu$ the find step. Note that it is possible to show that either $\lambda<.8$ or $\left|\lambda^{\prime}\right|<.8$.

When we find $\lambda$, we can put $\gamma_{g}=\lambda$ and $\gamma_{h}=\nu$ and then determine a new lattice $\left(1 / \gamma_{g}\right)$ (h) by the invert step of Section 4 . Notice that we can save some labour in the invert step if we retain some of the calculations performed in the find step. Note that it is possible to show that either $\lambda<.8$ or $\left|\lambda^{\prime}\right|<.8$.

Thus, we have an algorithm for finding $\gamma_{g}$ when $\rho^{2} /|f|>9.0003 D$. When $\rho^{2} /|f|<$ $9.0003 D$, we make use of the results of Section 7. We can find $\psi_{p}$ and $\phi_{p}$ by using algorithm $A$ of [18]. However, we have the same problem of precision in using $A$ as that mentioned in [18]; thus, we must modify this algorithm. We can not simply use the modification given in [18], because it was assumed throughout the discussion there that the only points of $R$ in $E_{1}$, were $(0,0,0)$ and $( \pm 1,0, \pm 1)$. This need no longer be true here. We also point out that, since $\lambda\left|\lambda^{\prime}\right|<.8$, no more than $O(\log D)$ pre-reduction steps need to be executed before we find that $\rho^{2} /|f|<9.0003 D$.

Let $\{1, \mu, \nu\}$ be a basis of the form (8.1) such that (8.2) is true. Since $\rho^{2} /|f|<$ $9.0003 D$ and $f \mid \rho$, we deduce that $|f| \leqslant \rho<9.0003 D, \rho /|f|<\sqrt{9.0003 D}$ and therefore

$$
\left|m_{2}\right|, \delta\left|n_{3}\right|, \delta\left|m_{3}\right|<\beta, \quad\left|n_{2}\right|<9.0003 D,
$$

where $\beta=3.002 \delta^{2}$.

For any $\Omega \in \mathcal{R}$, where $\Omega \approx \omega=\left(q_{1}+q_{2} \delta+q_{3} \delta^{2}\right) / \rho, q_{1}, q_{2}, q_{3} \in \mathcal{Z}$, define

$$
\begin{aligned}
& x_{\omega}=q_{2} I_{1}+\left[I_{1} \delta\right] q_{3}, \quad y_{\omega}=q_{2} I_{1}-\left[I_{1} \delta\right] q_{3}, \\
& \bar{x}_{\omega}=\left[3 \delta I_{2}\right] q_{2}+\left[3 \delta^{2} I_{2}\right] q_{3}, \quad \bar{y}_{\omega}=\left[\sqrt{3} \delta I_{2}\right] q_{2}-\left[\sqrt{3} \delta^{2} I_{2}\right] q_{3},
\end{aligned}
$$

where $I_{1}, I_{2} \in \mathscr{\Psi}$. We will specify values for $I_{1}$ and $I_{2}$ later.

We require

LEMmA 8.1. Suppose $\Omega \in G$ and $\left|q_{2}\right|+\left|q_{3}\right|<2 k$. Let $I_{2}>k / \rho(1 / 2-\sqrt{3} / 4)$, and let $\left(\xi_{\omega}, \eta_{\omega}\right)$ be the puncture of $\Omega$. If $\left|\bar{y}_{\omega}\right| \geqslant I_{2} \rho$, then $\left|\eta_{\omega}\right|>\sqrt{3} / 4$; if $\left|\bar{x}_{\omega}\right| \geqslant I_{2} \rho$, then $\left|\xi_{\omega}\right|>\sqrt{3} / 4$.

Proof. Since

$$
\begin{aligned}
\left|\eta_{\omega}-\bar{y}_{\omega} / 2 \rho I_{2}\right|,\left|\xi_{\omega}-\bar{x}_{\omega} / 2 \rho I_{2}\right| \\
\quad<\left(\left|q_{2}\right|+\left|q_{3}\right|\right) / 2 \rho I_{2}<k / \rho I_{2}<1 / 2-\sqrt{3} / 4,
\end{aligned}
$$

the result follows easily.

LEMMA 8.2. If the conditions of Lemma 8.1 hold, except that both $\left|\bar{y}_{\omega}\right|$ and $\left|\bar{x}_{\omega}\right|$ are now less than $I_{2} \rho$, then $\Omega^{*} \in \bigodot_{1}$.

Proof. From (8.7) we see that $\left|\eta_{\omega}\right|<1-\sqrt{3} / 4<\sqrt{3} / 2$ and $\left|\xi_{\omega}\right|<1-\sqrt{3} / 4$. Since $\xi_{\omega}^{2}+\eta_{\omega}^{2}<1$, we must have $\Omega^{*} \in \mathcal{C}_{1}$ by Lemma 7.4. 
Lemma 8.2 is an important result for the development of our algorithm for finding $\gamma_{g}$; for it allows us to assume that our current basis $\{1, \mu, \nu\}$ of $R$ is such that one of $\left|\xi_{\mu}\right|$ or $\left|\eta_{\mu}\right|$ must exceed $\sqrt{3} / 4$. If neither one of them, as determined by an examination of $\bar{y}_{\mu}$ and $\bar{x}_{\mu}$ exceeds $\sqrt{3} / 4$, then we can immediately send $\mu$ to the find step for the determination of a value for $\gamma_{g}$. We will now present several lemmas. As the methods of proof of these lemmas are very similar or identical to the methods used in proving analogous lemmas in Sections 7 and 8 of [18], we will only state these lemmas, except in those cases where there may be some differences in the proofs. In these cases, we will sketch the proof.

LEMMA 8.3. If $I_{1}>3.1(2 \beta)^{3} / \delta, \mu$ and $\nu$ are given in (8.1) such that (8.6) holds, then $\operatorname{sgn}\left(x_{\mu}\right)=\operatorname{sgn}\left(\xi_{\mu}\right), \operatorname{sgn}\left(y_{\mu}\right)=\operatorname{sgn}\left(\eta_{\mu}\right), \operatorname{sgn}\left(x_{\nu}\right)=\operatorname{sgn}\left(\xi_{\nu}\right), \operatorname{sgn}\left(y_{\nu}\right)=\operatorname{sgn}\left(\eta_{\nu}\right)$, $\operatorname{sgn}\left(\xi_{\nu}-\xi_{\mu}\right)=\operatorname{sgn}\left(x_{\nu}-x_{\mu}\right), \operatorname{sgn}\left(\eta_{\nu}-\eta_{\mu}\right)=\operatorname{sgn}\left(y_{\nu}-y_{\mu}\right)$.

LEMMA 8.4. Let $\left(\xi_{\pi}, \eta_{\pi}\right)$ be the puncture of $\Pi \in$ ? $R$ such that $0<\xi_{\pi}<\xi_{\mu},\left|\eta_{\pi}\right|$ $<\left|\eta_{\mu}\right|$, and let $\left(\xi_{\omega}, \eta_{\omega}\right)$ be the puncture of $\Omega \in$ o $R$. If $I_{1}>3.1(4 \beta)^{3} / \delta$ and $\left|\left[\xi_{\omega} / \xi_{\pi}\right]-\left[\eta_{\omega} / \eta_{\pi}\right]\right| \leqslant 3$, then $\left[\xi_{\omega} / \xi_{\pi}\right]=\left[x_{\omega} / x_{\pi}\right]$ and $\left[\eta_{\omega} / \eta_{\pi}\right]=\left[y_{\omega} / y_{\pi}\right]$.

Proof. Let $\Pi \approx \pi=\left(p_{1}+p_{2} \delta+p_{3} \delta^{2}\right) / \rho, \Omega \approx \omega=\left(q_{1}+q_{2} \delta+q_{3} \delta^{2}\right) / \rho, k_{1}=$ $\left[\xi_{\omega} / \xi_{\pi}\right], k_{2}=\left[\eta_{\omega} / \eta_{\pi}\right], t_{i}=q_{2}-k_{i} p_{2}, u_{i}=q_{3}-k_{i} p_{3}, \quad \xi_{i}=3 \delta\left(t_{i}+u_{i} \delta\right) / 2 \rho, \quad \eta_{i}$ $=\sqrt{3} \delta\left(t_{i}-u_{i} \delta\right) / 2 \rho(i=1,2)$. By using the reasoning of Lemma 7.8 of [18], we find that

$$
\begin{aligned}
& \left|\eta_{1}\right|,\left|\eta_{1}-\eta_{\pi}\right|<4\left|\eta_{\pi}\right| ; \quad\left|\xi_{1}\right|,\left|\xi_{1}-\xi_{\pi}\right|<\left|\xi_{\pi}\right| \\
& \left|\eta_{2}\right|,\left|\eta_{2}-\eta_{\pi}\right|<\left|\eta_{\pi}\right| ; \quad\left|\xi_{2}\right|,\left|\xi_{2}-\xi_{\pi}\right|<4\left|\xi_{\pi}\right| .
\end{aligned}
$$

It follows that $\left|\delta u_{i}\right|<4 \beta,\left|\delta\left(u_{i}-p_{3}\right)\right|<4 \beta, \delta\left|p_{3}\right|<4 \beta(i=1,2)$; hence the lemma follows from Lemma 7.5 of [18].

LEMMA 8.5. Let $\Pi$ and $\Omega$ be as defined in Lemma 8.4, and suppose that $I_{1}>$ $3.1 \delta^{2}(2 \sqrt{3} \delta+\beta / \delta)^{3}$. We must have $\left[x_{\omega} / x_{\pi}\right]=\left[\xi_{\omega} / \xi_{\pi}\right]$ when $\xi_{\pi}>\sqrt{3} / 4$ and $\left[y_{\omega} / y_{\pi}\right]=\left[\eta_{\omega} / \eta_{\pi}\right]$ when $\left|\eta_{\pi}\right|>\sqrt{3} / 4$.

LEMMA 8.6. Let $\Pi$ and $\Omega$ be defined as in Lemma 8.4 and suppose that $\{1, \pi, \omega\}$ is a basis of R. If $I_{1}>3.1(4 \beta)^{3} / \delta$ and $\left|\left[x_{\omega} / x_{\pi}\right]-\left[y_{\omega} / y_{\pi}\right]\right| \leqslant 1$, then $\left[\xi_{\omega} / \xi_{\pi}\right]=\left[x_{\omega} / x_{\pi}\right]$ and $\left[\eta_{\omega} / \eta_{\pi}\right]=\left[y_{\omega} / y_{\pi}\right]$.

Proof. We note that the Eqs. (7.2), (7.3), and (7.4) of [18] hold with $e_{r}$ replaced by $f$. By using the facts that

$$
\left|p_{2}\right|+\left|p_{3}\right| \delta<2 \beta, \quad|f|<9.0003 D \text { and } 4 \beta\left(2 \beta^{2} / \delta+9.0003 D\right)<3.1(4 \beta)^{3} / \delta,
$$

we can show by the methods of Lemma 7.10 of [13] that

$$
\left|p_{2}+p_{3} \delta\right|,\left|p_{2}-p_{3} \delta\right|>\delta|f| / 3 \beta ; \quad\left|x_{\pi}\right|,\left|y_{\pi}\right|>\frac{I_{1} \delta|f|}{3 \beta}-\beta .
$$

Thus, we can find that $\left|x_{\pi}\right|\left|p_{2}+p_{3} \delta\right|,\left|y_{\pi}\right|\left|p_{1}-p_{3} \delta\right|>2|f|$, and therefore

$$
\left|\xi_{\omega} / \xi_{\pi}-x_{\omega} / x_{\pi}\right|,\left|\eta_{\omega} / \eta_{\pi}-y_{\omega} / y_{\pi}\right|<1 / 2 \text {. }
$$

Since $\left|\left[x_{\omega} / x_{\pi}\right]-\left[y_{\omega} / y_{\pi}\right]\right| \leqslant 1$, it follows that

$$
\left|\left[\xi_{\omega} / \xi_{\pi}\right]-\left[\eta_{\omega} / \eta_{\pi}\right]\right| \leqslant 3
$$

and we have the result from Lemma 8.4. 
Lemma 8.7. Let $\Pi$ and $\Omega$ be defined as in Lemma 8.4, and suppose that $\{1, \pi, \omega\}$ is a basis of $\left(R\right.$ and one of $\xi_{\pi}$ or $\left|\eta_{\pi}\right|$ exceeds $\sqrt{3} / 4$. If $I_{1}>3.1\left(4 \beta^{3}\right) / \delta$ and $j=\left[x_{\omega} / x_{\pi}\right]-$ $\left[y_{\omega} / y_{\pi}\right]$, then $j$ and $\left[\xi_{\omega} / \xi_{\pi}\right]-\left[\eta_{\omega} / \eta_{\pi}\right]$ have the same sign.

Proof. We use the result that

$$
\xi_{\omega} \eta_{\pi}-\eta_{\omega} \xi_{\pi}=3 \sqrt{3} D f / 2 \rho^{2}
$$

and the method of Lemma 7.11 of [18].

Let $K_{1}(a, b), K_{2}(a, b), K_{3}(a, b)$ denote, respectively, the matrices

$$
\left(\begin{array}{ll}
a & 1 \\
b & 0
\end{array}\right), \quad\left(\begin{array}{ll}
1 & a \\
0 & b
\end{array}\right), \quad\left(\begin{array}{ll}
0 & a \\
1 & b
\end{array}\right)
$$

Let $I_{1}>3.1(4 \beta)^{3} / \delta, I_{2}>2(3 \beta)^{3} / \sqrt{3} \delta$. We now give that part of the reduction algorithm that we call

\section{Algorithm R 1}

(i) Transform the basis $\{1, \mu, \nu\}$ of (8.1) by $\left(\begin{array}{cc}k_{1} & 0 \\ 0 & k_{2}\end{array}\right)$, where $k_{1}=\operatorname{sgn}\left(x_{\mu}\right), k_{2}=$ $\operatorname{sgn}\left(x_{\nu}\right)$.

(ii) If $x_{\nu}>x_{\mu}$ go to (iii); otherwise, transform the basis by $K_{2}(1,1)$ and go to (iii) unless $y_{\nu} y_{\mu}<0$ and $\left|y_{\nu}\right|>\left|y_{\mu}\right|$. If this latter case occurs, transform the basis by $K_{1}(0,1)$ instead of $K_{2}(1,1)$ and then go to (v).

(iii) If $y_{\nu} y_{\mu}<0$ go to (iv); otherwise

(1) If $\left[y_{\nu} / y_{\mu}\right]=\left[x_{\nu} / x_{\mu}\right]=k$, transform the basis by $K_{1}(-k, 1)$ until $\left[y_{\nu} / y_{\mu}\right] \neq$ $\left[x_{\nu} / x_{\mu}\right]$. When such a basis is found, execute one of the following steps.

(2) If $\left[x_{\nu} / x_{\mu}\right]+1=\left[y_{\nu} / y_{\mu}\right]=k$, transform the basis by $K_{1}(k,-1)$ and go to (iv).

(3) If $k=\left[x_{\nu} / x_{\mu}\right]=\left[y_{\nu} / y_{\mu}\right]+1$, transform the basis by $K_{1}(-k, 1)$ and go to

(4) If $\left[x_{\nu} / x_{\mu}\right]<\left[y_{\nu} / y_{\mu}\right]-1$, execute the find step and terminate R1 when $\left|\bar{y}_{\mu}\right|$ and $\bar{x}_{\mu}<I_{2} \rho$. If $\left|\bar{y}_{\mu}\right| \geqslant I_{2} \rho$, transform the basis by $K_{2}\left(\left[y_{\nu} / y_{\mu}\right],-1\right)$ and go to (v); if $\left|\bar{y}_{\mu}\right|<I_{2} \rho$ and $\bar{x}_{\mu} \geqslant I_{2} \rho$ transform the basis by $K_{1}\left(\left[x_{\nu} / x_{\mu}\right]+1,-1\right)$ and go to (v).

(5) If $\left[x_{\nu} / x_{\mu}\right]>\left[y_{\nu} / y_{\mu}\right]+1$, execute the find step and terminate $\mathrm{R} 1$ when $\left|\bar{y}_{\mu}\right|, \bar{x}_{\mu}<I_{2} \rho$. If $\left|\bar{y}_{\mu}\right| \geqslant I_{2} \rho$, transform the basis by $K_{2}\left(-\left[y_{\nu} / y_{\mu}\right]-1,1\right)$ and go to $(\mathrm{v})$; if $\left|y_{\mu}\right|<I_{2} \rho$ and $\bar{x}_{\mu} \geqslant I_{2} \rho$, transform the basis by $K_{1}\left(-\left[x_{\nu} / x_{\mu}\right], 1\right)$ and go to $(\mathrm{v})$.

(iv) If $\left|y_{\mu}\right|>\left|y_{\nu}\right|$, go to (v). If $\left|y_{\mu}\right| \leqslant\left|y_{\nu}\right|$ and $\left|\bar{y}_{\mu}\right|, \bar{x}_{\mu}<I_{2} \rho$, execute the find step and terminate R1. If $\left|\bar{y}_{\mu}\right| \geqslant I_{2} \rho$, transform the basis by $K_{2}\left(\left[-y_{\nu} / y_{\mu}\right], 1\right)$ and go to (v); if $\left|\bar{y}_{\mu}\right|<I_{2} \rho$ and $\bar{x}_{\mu} \geqslant I_{2} \rho$, transform the basis by $K_{1}\left(-\left[x_{\nu} / x_{\mu}\right], 1\right)$ and go to $(\mathrm{v})$.

(v) If $\left|\bar{y}_{\nu}\right| \leqslant I_{2} \rho$ and $\left|\bar{y}_{\mu}\right| \geqslant I_{2} \rho$, terminate algorithm R1. If $\bar{x}_{\mu}<I_{2} \rho$ and $\left|\bar{y}_{\mu}\right|<I_{2} \rho$, execute the find step and terminate $\mathrm{R} 1$; otherwise, go to (vi).

(vi)(1) If $\left|\bar{y}_{\nu}\right|>I_{2} \rho$, transform the basis by $K_{3}(1, k)$, where $k=\left[-y_{\mu} / y_{\nu}\right]$ and go to (v). If $\left|\bar{y}_{\nu}\right| \leqslant I_{2} \rho$, go to (2).

(2) If $\left|\bar{y}_{\mu}\right| \leqslant I_{2} \rho$, transform the basis by $K_{1}(-k, 1)$, where $k=\left[x_{\nu} / x_{\mu}\right]$ and go to (v).

THEOREM 8.8. Suppose that we execute algorithm R1 up to the beginning of step (v), and suppose further that it was not necessary, during this process, to go to the find step. If $\{1, \kappa, \lambda\}$ is the basis of $R$ that the algorithm has produced up to the beginning of step 
(v), then we must have

$$
\xi_{\lambda}>\xi_{\kappa}>0, \quad\left|\eta_{\kappa}\right|>\left|\eta_{\lambda}\right|, \quad \eta_{\lambda} \eta_{\kappa}<0,
$$

where $\left(\xi_{\lambda}, \eta_{\lambda}\right)$ and $\left(\xi_{\kappa}, \eta_{\kappa}\right)$ are, respectively, the punctures of $\Lambda(\approx \lambda)$ and $K(\approx \kappa)$.

Proof. Analogous to that of Theorem 7.12 of [18]. We use $\rho$ instead of $\sigma_{r}$ and $\beta$ instead of $(1+\sqrt{5}) \delta^{2}$.

Lemma 8.9. Let $\kappa, \lambda$ be as described in Theorem 8.7. We must have

$$
\xi_{\lambda} / 3, \xi_{\kappa} / 3,\left|\eta_{\lambda}\right| / / \overline{3},\left|\eta_{\kappa}\right| / \overline{1}<3 \beta \delta / 2 \rho \text {. }
$$

Proof. Let $\{1, \mu, \nu\}=\left\{1,\left(m_{1}+m_{2} \delta+m_{3} \delta^{2}\right) / \rho,\left(n_{1}+n_{2} \delta+n_{3} \delta^{2}\right) / \rho\right\}$ be the new basis which results after step (i) of Rl has been executed. Note that (8.6) is still true. If $\{1, \bar{\mu}, \bar{\nu}\}$ is the basis produced from $\{1, \mu, \nu\}$ after step (ii) of $\mathrm{R} 1$ has been executed, by Lemma 8.3 we have 3 possible cases:

$$
\begin{gathered}
\left\{\begin{array}{l}
\bar{\mu}=\mu \\
\bar{\nu}=\nu
\end{array} \text { when } \xi_{\nu}>\xi_{\mu} ;\right. \\
\left\{\begin{array}{l}
\bar{\mu}=\nu \\
\bar{\nu}=\mu
\end{array} \text { when } \xi_{\nu}<\xi_{\mu},\left|\eta_{\nu}\right|>\left|\eta_{\mu}\right|, \eta_{\nu} \eta_{\mu}<0 ;\right. \\
\left\{\begin{array}{l}
\bar{\mu}=\mu \\
\bar{\nu}=\mu+\nu
\end{array} \text { when } \xi_{\nu}<\xi_{\mu} \text { and either } \eta_{\nu} \eta_{\mu}>0 \text { or }\left|\eta_{\nu}\right|<\left|\eta_{\mu}\right| .\right.
\end{gathered}
$$

In the third of these cases, we see that

$$
\eta_{\bar{\nu}} \eta_{\bar{\mu}}=\eta_{\mu}^{2}+\eta_{\mu} \eta_{\nu}
$$

If $\eta_{\mu} \eta_{\nu}>0$, then $\eta_{\bar{\nu}} \eta_{\bar{\mu}}>0$. If $\eta_{\mu} \eta_{\nu}<0$, then $\left|\eta_{\nu}\right|<\left|\eta_{\mu}\right|$ and

$$
\eta_{\bar{\nu}} \eta_{\bar{\mu}}=\left|\eta_{\mu}\right|\left(\left|\eta_{\mu}\right|-\left|\eta_{\nu}\right|\right)>0
$$

That is, in this case, one of the substeps of step (iii) of Rl will be executed next. Thus, if we arrive at the beginning of step ( $v$ ) by skipping over all the substeps of (iii) and step (iv), then we must have $\lambda=\mu, \kappa=\nu$ or $\lambda=\nu, \kappa=\mu$.

Suppose that this is how we did arrive at the beginning of step (v), and suppose further that $\lambda=\mu$ and $\kappa=\nu$. Then $\xi_{\kappa} / 3<\xi_{\lambda} / 3=\xi_{\mu} / 3<\delta(2 \beta) / 2 \rho$ and $\left|\eta_{\kappa}\right|>$ $\left|\eta_{\lambda}\right|$. If $\xi_{\kappa}<1 / 3$, then $\left|n_{2}+n_{3} \delta\right|<2 \rho / 9 \delta$. Since $\left|n_{3} \delta\right|<\beta$ and $3 \beta \delta>\rho$, we find that $\left|n_{2}-n_{3} \delta\right|<2 \rho / 9 \delta+2 \beta$ and $\left|\eta_{\kappa}\right| / \sqrt{3}=\left|\eta_{\nu}\right| / \sqrt{3}<3 \delta \beta / 2 \rho$. If $\xi_{\kappa}>1 / 3$, we use the result

$$
\left|\xi_{\lambda} \eta_{\kappa}-\xi_{\kappa} \eta_{\lambda}\right|=3 \sqrt{3} D|f| / 2 \rho^{2}
$$

together with the facts $\eta_{\kappa} \eta_{\lambda}<0$ and $\xi_{\lambda}>\xi_{\kappa}>1 / 3$, to show that

$$
\left|\eta_{\kappa}\right| / \sqrt{3}<3 D f / 2 \rho^{2} \xi_{\lambda}<9 D|f| / 2 \rho^{2}<3 \beta \delta / 2 \rho .
$$

If, instead, we have $\lambda=\nu, \kappa=\mu$ at the beginning of step (v), we can show the truth of the lemma by using a similar argument. The only significant change in the proof is that we first assume $\left|\eta_{\lambda}\right|<1 / 2 \sqrt{3}$ and then assume $\left|\eta_{\lambda}\right|>1 / 2 \sqrt{3}$.

If we arrive at the beginning of step (v) by executing one of the substeps of (iii) or step (iv), then we must execute one of substep 4 or substep 5 of (iii) or step (iv). Let $\{1, \bar{\kappa}, \bar{\lambda}\}$ be the basis of $\mathrm{R} 1$ which we have obtained from $\mathrm{R} 1$ just before either of 
(iii) -4 , (iii) -5 or (iv) is executed. If $\left(\xi_{\bar{\kappa}}, \eta_{\bar{\kappa}}\right)$ is the puncture of $\bar{K}(\approx \bar{\kappa})$, we certainly have

$$
\left|\xi_{\bar{\kappa}}\right| / 3, \quad\left|\eta_{\bar{\kappa}}\right| / 1 \overline{3}<\delta \beta / \rho .
$$

If in any of the above steps ((iii)-4, (iii) -5 , or (iv)), we start with $\left|\bar{y}_{\bar{\kappa}}\right| \geqslant I_{2} \rho$, then a transformation of the type $K_{2}(a, b)$ is used and $\kappa=\bar{\kappa}$. It follows that $\left|\eta_{\kappa}\right|>\sqrt{3} / 4$ and from (8.8)

$$
\xi_{\lambda} / 3<2 D|f| / \rho^{2}<\beta \delta / \rho
$$

Also, $\left|\eta_{\lambda}\right| / \sqrt{3}<\left|\eta_{\kappa}\right| / \sqrt{3}<\delta \beta / \rho$.

If we start with $\left|\bar{x}_{\bar{\kappa}}\right| \geqslant I_{2} \rho$, then a transformation of the type $K_{1}(a, b)$ is used and we get $\lambda=\bar{\kappa}$. Thus, $\xi_{\kappa}>\sqrt{3} / 4$ and $\left|\eta_{\kappa}\right| / \sqrt{3}<3 \beta \delta / 2 \rho$. The rest of the results follow from the facts $\lambda=\bar{\kappa}$ and $\xi_{\lambda}>\xi_{\kappa}$.

LEMMA 8.10. Let $\{1, \mu, \nu\}$ be any basis of sh such that $\xi_{\nu}>\xi_{\mu}>0,\left|\eta_{\mu}\right|>\left|\eta_{\nu}\right|$ and $\eta_{\nu} \eta_{\mu}<0$. If $\left|\eta_{\mu}\right|>\sqrt{3} / 4,\left|\bar{y}_{\nu}\right| \geqslant I_{2} \rho,\left|\eta_{\nu}\right| / \sqrt{3}<3 \beta \delta / 2 \rho$, then $\left|\eta_{\nu}\right|>\sqrt{3} / 4,\left|n_{2}\right|+$ $\left|n_{3}\right| \delta<3 \beta$ and $\left|n_{2}\right|,\left|\delta n_{3}\right|<8 \delta^{2}$; if $\xi_{\nu}>\sqrt{3} / 4, \bar{x}_{\mu} \geqslant I_{2} \rho, \xi_{\mu} / 3<3 \beta \delta / 2 \rho$, then $\xi_{\mu}>\sqrt{3} / 4,\left|m_{2}\right|+\left|m_{3}\right| \delta<3 \beta$ and $\left|m_{2}\right|,\left|\delta m_{3}\right|<8 \delta^{2}$.

Proof. We prove the last part only; the proof of the first part is similar. Since $\xi_{\nu}$ $>\sqrt{3} / 4$, we have

$$
\left|\eta_{\mu}\right|<\frac{4}{\sqrt{3}} \cdot \frac{3 \sqrt{3} D|f|}{2 \rho^{2}} \text { and }\left|m_{2}-m_{3} \delta\right|<\frac{12 \delta^{2}}{\sqrt{3}}
$$

Since $\left|m_{2}+m_{3} \delta\right|<(3 \beta \delta / 2 \rho)(2 \rho / \delta)=3 \beta \quad\left(>12 \delta^{2} / \sqrt{3}\right)$, we see that $\left|m_{2}\right|+$ $\left|m_{3} \delta\right|<3 \beta$. Also, $\left|m_{2}\right|,\left|\delta m_{3}\right|<1 / 2\left(3 \beta+12 \delta^{2} / \sqrt{3}\right)<8 \delta^{2}$. By Lemma 8.1, we have $\xi_{\mu}>\sqrt{3} / 4$.

We are now able to present the main result of this section.

THEOREM 8.11. Algorithm R1 either executes the find step before it terminates or it terminates with a basis $\{1, \psi, \phi\}$ of $h$ such that

$$
\begin{gathered}
\xi_{\phi}>\xi_{\psi}>0, \quad\left|\eta_{\phi}\right|<\left|\eta_{\psi}\right|, \quad \eta_{\phi} \eta_{\psi}<0, \\
\left|\eta_{\phi}\right|<1-\sqrt{3} / 4, \quad\left|\eta_{\psi}\right|>\sqrt{3} / 4, \quad\left|\eta_{\psi}\right|>\left(1-\left|\eta_{\phi}\right|\right) / 2 .
\end{gathered}
$$

Proof. Let $\{1, \mu, \nu\}$ be the basis of $R$ which algorithm R1 has produced up to the beginning of step $(\mathrm{v})$, but before step $(\mathrm{v})$ is ever executed. By Lemma 8.9, we have $\left|m_{2}\right|+\left|m_{3}\right| \delta<3 \beta,\left|n_{2}\right|+\left|n_{3}\right| \delta<3 \beta$. Thus, if $\left|\bar{y}_{\nu}\right| \leqslant I_{2} \rho$ and $\left|\bar{y}_{\mu}\right| \geqslant I_{2} \rho$, we know from Lemma 8.1 that $\left|\eta_{\nu}\right|<1-\sqrt{3} / 4$ and $\left|\eta_{\mu}\right|>\sqrt{3} / 4$. Also, if $\bar{x}_{\mu}<I_{2} \rho$ and $\left|\bar{y}_{\mu}\right|<I_{2} \rho$, then $\left|\xi_{\mu}\right|,\left|\eta_{\nu}\right|<1-\sqrt{3} / 4$ and the find step will obtain a value of $\gamma_{g}$. Suppose that we have $\left|\bar{y}_{\mu}\right|<I_{2} \rho, \bar{x}_{\mu} \geqslant I_{2} \rho$ and $\left|\bar{y}_{\nu}\right|<I_{2} \rho$. We must go to substep (vi)-2. Since $\left|m_{2}\right|+\left|m_{3} \delta\right|<3 \beta$, we have $\xi_{\nu}>\xi_{\mu}>\sqrt{3} / 4$ by Lemma 8.1 and Theorem 8.8. Hence, $\left|m_{2}+m_{3} \delta\right|>\rho / 2 \sqrt{3} \delta$ and

$$
|f| /\left|m_{2}+m_{3} \delta\right|+\left|m_{3}\right|<2 \sqrt{3} \delta+8 \delta
$$

by Lemma 8.10 . Since $I_{1}>3.1 \delta^{2}(2 \sqrt{3} \delta+8 \delta)^{3}$, we must have $\left[x_{\nu} / x_{\mu}\right]=\left[\xi_{\nu} / \xi_{\mu}\right]$ by Corollary 7.5.1 of [18]. If we put $\eta_{\mu}^{(1)}=\eta_{\nu}, \eta_{\mu}^{(2)}=\eta_{\mu}, \xi_{\mu}^{(1)}=\xi_{\nu}, \xi_{\mu}^{(2)}=\xi_{\mu}, \mu^{(1)}=\nu$, $\mu^{(2)}=\mu, k_{i}=\left[x_{\mu}^{(i)} / x_{\nu}^{(i+1)}\right]$, we see, by Lemma 8.10, that as long as $\bar{x}_{\mu}^{(i)} \geqslant I_{2} \rho$ and 
$\left|\bar{y}_{\mu}^{(i)}\right|<I_{2} \rho$ for $i=1,2,3, \ldots, j$, substep 2 of (vi) will generate two sequences

$$
\begin{aligned}
& \eta_{\mu}^{(1)}, \eta_{\mu}^{(2)}, \eta_{\mu}^{(3)}, \ldots \eta_{\mu}^{(j)}, \\
& \xi_{\mu}^{(1)}, \xi_{\mu}^{(2)}, \xi_{\mu}^{(3)} \ldots \ldots \xi_{\mu}^{(j)},
\end{aligned}
$$

such that $\eta_{\mu}^{(i+1)}=-k_{i} \eta_{\mu}^{(i)}+\eta_{\mu}^{(i-1)}, \xi_{\mu}^{(i+1)}=-k_{i} \xi_{\mu}^{(i)}+\xi_{\mu}^{(i-1)}, \mu^{(i+1)}=-k_{i} \mu^{(i)}+\mu^{(i-1)}$.

Also

$$
\begin{gathered}
\left|\eta_{\mu}^{(1)}\right|<\left|\eta_{\mu}^{(2)}\right|<\left|\eta_{\mu}^{(3)}\right|<\cdots<\left|\eta_{\mu}^{(j)}\right|, \\
\xi_{\mu}^{(1)}>\xi_{\mu}^{(2)}>\xi_{\mu}^{(3)}>\cdots>\xi_{\mu}^{(1)},
\end{gathered}
$$

$k_{i}=\left[\xi_{\mu}^{(i)} / \xi_{\mu}^{(i+1)}\right]$ and $\eta_{\mu}^{(i)} \eta_{\mu}^{(i-1)}<0, i=2,3, \ldots, j$.

We also see that if $m_{2}^{(i)}+m_{3}^{(i)} \delta=2 \rho \xi_{\mu}^{(i)} / 3 \delta$, then $\left|m_{2}^{(i)}\right|+\delta\left|m_{3}^{(i)}\right|<3 \beta$ for $i=$ $1,2,3, \ldots, j$ (Lemma 8.10 ). Since there are only a finite number of possibilities for $\left(m_{2}^{(i)}, m_{3}^{(i)}\right)$, there must be a finite value for $j$ such that either $\bar{x}_{\mu}^{(j)}<I_{2} \rho$ or $\left|\bar{y}_{\mu}^{(j)}\right| \geqslant I_{2} \rho$. If $\bar{x}_{\mu}^{(j)}<I_{2} \rho$ and $\left|\bar{y}_{\mu}^{(j)}\right|<I_{2} \rho$, then since $\xi_{\mu}^{(j)}>0$, we have $0<\xi_{\mu}^{(j)}<1-$ $\sqrt{3} / 4$ by $(8.7)$ and $\left|\eta_{\mu}^{(j)}\right|<1-\sqrt{3} / 4$ and we send the basis $\left\{1, \mu^{(j)}, \mu^{(j-1)}\right\}$ to the find step. If $\left|\bar{y}_{\mu}^{(j-1)}\right|<I_{2} \rho$ and $\left|\bar{y}_{\mu}^{(j)}\right| \geqslant I_{2} \rho$, then we have $\{1, \phi, \psi\}=\left\{1, \mu^{(j)}, \mu^{(j-1)}\right\}$. By Lemma 8.1, we have $\left|\eta_{\psi}\right|>\sqrt{3} / 4,\left|\eta_{\phi}\right|<1-\sqrt{3} / 4$. Also, if

$$
\psi=\left(t_{1}+t_{2} \delta+t_{3} \delta^{2}\right) / \rho, \quad \phi=\left(s_{1}+s_{2} \delta+s_{3} \delta^{2}\right) / \rho,
$$

then

$$
\left|\eta_{\psi}\right|=\left|\bar{y}_{\psi}\right| / 2 \rho I_{2}+\alpha,
$$

where $\alpha<\left(\left|t_{2}\right|+\left|t_{3}\right|\right) / 2 \rho I_{2}<3 \beta / 2 \rho I_{2}$. Also, $\left|\eta_{\phi}\right| / 2=\sqrt{3} \delta\left|s_{2}-s_{3} \delta\right| / 4 \rho$ $>\sqrt{3} \delta / 4 \rho\left(\left|s_{2}\right|+\left|s_{3}\right| \delta\right)^{2}$ by Lemma 7.1 of [18]. Hence

$$
\left|\eta_{\phi}\right| / 2>\sqrt{3} \delta / 4 \rho(3 \beta)^{2}>3 \beta / 2 \rho I_{2}>|\alpha|
$$

and

$$
\left|\eta_{\psi}\right|>\left(1-\left|\eta_{\phi}\right|\right) / 2
$$

The proof of the theorem for the case in which we use substep 1 of (iv) instead of substep 2 follows by using similar reasoning. (See Theorem 8.3 of [18].)

9. Determination of $\gamma_{g}$. As we have seen in Section 8, we have an algorithm R1 which either finds a value $\gamma_{g}$ or obtains $\phi$ and $\psi$ such that (7.3) holds. We will now show how we can obtain a value for $\gamma_{g}$ (if one exists) when we know $\phi$ and $\psi$.

We first put

$$
\begin{array}{ll}
X_{\psi}=t_{2}\left[I_{3} \delta\right]+t_{3}\left[I_{3} \delta^{2}\right], & Y_{\psi}=t_{2}\left[I_{3} \delta\right]-t_{3}\left[I_{3} \delta^{2}\right], \\
X_{\phi}=s_{2}\left[I_{3} \delta\right]+s_{3}\left[I_{3} \delta^{2}\right], & Y_{\phi}=s_{2}\left[I_{3} \delta\right]-s_{3}\left[I_{3} \delta^{2}\right],
\end{array}
$$

where $I_{3} \in \mathscr{Z}$. Since

$$
-\left|t_{2}\right|-\left|t_{3}\right|+\left|\frac{2 \rho}{\sqrt{3}} I_{3} \eta_{\psi}\right|<\left|Y_{\psi}\right|<\left|\frac{2 \rho}{\sqrt{3}} I_{3} \eta_{\psi}\right|+\left|t_{2}\right|+\left|t_{3}\right|,
$$

we see that $\left|\eta_{\psi}\right|<\sqrt{3} / 2$ when $\left|Y_{\psi}\right|<\rho I_{3}-\left|t_{2}\right|-\left|t_{3}\right|$. Also, if

$$
\left(\left|t_{2}\right|+\left|t_{3}\right|\right) \sqrt{3} / 2 I_{3} \rho<.03
$$


then $\left|\eta_{\psi}\right|<1.19$ when $5\left|Y_{\psi}\right|<6 \rho I_{3}$ and $\left|\eta_{\psi}\right|>1$ when $5\left|Y_{\psi}\right|>6 \rho I_{3}$. Thus, if $I_{3}>$ $100 \beta$, we see by Theorem 7.7 and its corollaries that we have the possibilities shown in Table 2 for the set $Q$ of Section 7.

TABLE 2

\begin{tabular}{|l|c|}
\hline Restrictions $Y_{\psi}$ & \multicolumn{1}{|}{$Q$} \\
\hline$\left|Y_{\psi}\right|<\rho I_{3}-\left|t_{2}\right|-\left|t_{3}\right|$ & $\{(1,0),(0,1),(1,-1)\}$ \\
\hline $5\left|Y_{\psi}\right|<6 \rho I_{3}$ & $\{(1,0),(0,1),(1,-1),(1,1)\}$ \\
\hline $5\left|Y_{\psi}\right|>6 \rho I_{3}$ & $\{(1,0),(1,-1),(1,1),(2,1)\}$ \\
\hline
\end{tabular}

Let $\left|\mathcal{G}^{\prime}\right|=k$, and put $q_{i}=a_{i} s_{1}+b_{i} t_{1}, X_{i}=a_{i} X_{\phi}+b_{i} X_{\psi}, Y_{i}=a_{i} Y_{\phi}+b_{i} Y_{\psi}, d_{i}=$ $\left|a_{i} s_{2}+b_{i} t_{2}\right|+\left|a_{i} s_{3}+b_{i} t_{3}\right|<9 \beta, \omega_{i}=a_{i} \phi+b_{i} \psi$ for $\left(a_{i}, b_{i}\right) \in \mathcal{Q}, 1 \leqslant i \leqslant k$. If $\gamma_{g}$ exists, by Theorem 7.7, it must have the form $l+\omega_{i}, l \in \mathscr{Z}$ and $1 \leqslant i \leqslant k$. We first note that if

$$
\left|Y_{i}\right|>\left[\frac{2 \sqrt{3}}{2} \rho I_{3}\right]+1+d_{i},
$$

then $\left|\eta_{\omega_{1}}\right|>1$ and $\gamma_{g} \neq \omega_{i}+l$. Thus, if $(9.1)$ is true, we can eliminate $\left(a_{i}, b_{i}\right)$ from $Q$ and decrease $k$ by 1 . Also, if $(9.1)$ is not true, then

$$
\left|\eta_{\omega_{1}}\right|<2 \text { when } I_{3}>\sqrt{3} / 2\left(d_{i}+1\right) / \rho>9 \beta .
$$

We now require

Lemma 9.1. Let $\Omega(\approx \omega) \in \mathcal{R}$, where $\omega=\left(q_{1}+q_{2} \delta+q_{3} \delta^{2}\right) / \rho$, and let $\bar{\Omega}(\approx \bar{\omega})$, where $\bar{\omega}=l+\omega$ and $l \in \mathscr{Z}$. Put $I_{3}>d=\left|q_{2}\right|+\left|q_{3}\right|, X=q_{2}\left[I_{3} \delta\right]+q_{3}\left[I_{3} \delta^{2}\right], j=$ $\left[X / I_{3}\right], l_{1}=\left[\left(j-2 q_{1}\right) / 2 \rho\right], l_{2}=l_{1}+1$. If $j I_{3}-X>-d$ and $2 \rho \mid j-2 q_{1}$, put $l_{3}=$ $l_{1}-1$ and $u=3$; if $j I_{3}-X<d-I_{3}$ and $2 \rho \mid j+1-2 q_{1}$, put $l_{3}=l_{1}+2$ and $u=3$; otherwise, put $u=2$. If $\left|\zeta_{\bar{\omega}}\right|<1$, we must have $l \in\left\{l_{i} \mid 1 \leqslant i \leqslant u\right\}$. Further, if $l \in\left\{l_{i} \mid 1 \leqslant i \leqslant u\right\}$, then $\left|\zeta_{\bar{\omega}}\right|<2$.

Proof. If $\left|\zeta_{\bar{\omega}}\right|<1$, then

$$
-1<l+\left(2 q_{1}-q_{2} \delta-q_{3} \delta^{2}\right) / 2 \rho<1 .
$$

Now $q_{2} \delta I_{3}+q_{3} \delta^{2} I_{3}=X+\alpha$, where $|\alpha|<d$; hence,

$$
\begin{aligned}
-1+\left(X / I_{3}-2 q_{1}\right) & / 2 \rho+\alpha / 2 \rho I_{3}<l \\
& <1+\left(X / I_{3}-2 q_{1}\right) / 2 \rho+\alpha / 2 \rho I_{3} .
\end{aligned}
$$

Let $j-2 q_{1}=2 \rho l_{1}+r$, where $0 \leqslant r \leqslant 2 \rho-1$. Since $\left[\left(X / I_{3}-2 q_{1}\right) / 2\right]=$ $\left[\left(j-2 q_{i}\right) / 2 \rho\right]=l_{1}$, we have

$$
0<\left(X-2 q_{1} I_{3}\right) / 2 \rho I_{3}-l_{1}=\left(X-I_{3} j+r I_{3}\right) / 2 \rho I_{3}<1
$$

and

$$
\begin{aligned}
-1+\alpha / 2 \rho I_{3}+ & \left(X-I_{3} j+r I_{3}\right) / 2 \rho I_{3}<l-l_{1} \\
& <1+\alpha / 2 \rho I_{3}+\left(X-I_{3} j+r I_{3}\right) / 2 \rho I_{3} .
\end{aligned}
$$

Hence $-1 \leqslant l-l_{1} \leqslant 2$. If $l=l_{1}+2$, we must have

$$
\alpha / 2 \rho I_{3}+\left(X-I_{3} j+r I_{3}\right) / 2 \rho I_{3} \geqslant 1 \text {. }
$$


Since $0 \leqslant\left(X-I_{3} j\right) / 2 \rho I_{3}<1 / 2$, we see that this can happen only when $r=2 \rho-1$. In this case we must also have $X-I_{3} j+\alpha \geqslant I_{3}$ and therefore $X-I_{3} j+d>I_{3}$. Similarly, we can show that if $l=l_{1}-1$, then $r=0$ and $I_{3} j-X>-d$.

On the other hand, if $\bar{\omega}=\omega+l_{i}=l_{1}+\kappa+\omega(1 \leqslant i \leqslant u)$, then

$$
\begin{aligned}
\zeta_{\bar{\omega}} & =\kappa / 2 \rho+\left(2 \rho l_{1}+2 q_{1}-q_{2} \delta-q_{3} \delta^{2}\right) / 2 \rho \\
& =\kappa / 2 \rho-\alpha / 2 \rho I_{3}-\left(X-I_{3} j+r I_{3}\right) / 2 \rho I_{3} .
\end{aligned}
$$

Since $\left|\kappa / 2 \rho-\alpha / 2 \rho I_{3}-\left(X-I_{3} j+r I_{3}\right) / 2 \rho I_{3}\right|<2$, we see that $\left|\zeta_{\bar{\omega}}\right|<2$.

We now let $l_{i r}\left(1 \leqslant r \leqslant u_{i}\right)$ be the values of $l_{r}$, specified by Lemma 9.1 for each $\omega_{\text {, }}$ defined above. Put $q_{i r}=q_{i}+\rho l_{\imath r}, \Omega_{t r} \approx \omega_{\imath r}=l_{t r}+\omega_{i}$, where $1 \leqslant i \leqslant k, 1 \leqslant r \leqslant u_{\text {, }}$ and let

$$
\begin{aligned}
& \forall \|=\left\{I_{3} q_{i r}+X_{i} \mid 1 \leqslant i \leqslant k, 1 \leqslant r \leqslant u_{1}\right\}, \\
& \because=\left\{\Omega_{\imath r} \mid 1 \leqslant i \leqslant k, 1 \leqslant r \leqslant u_{1}\right\} .
\end{aligned}
$$

We prove

Lemma 9.2. If $I_{3}>487 \beta \sqrt{D}$ and $W_{\text {ir }}$ is the least element of "ill such that $\Omega_{\text {"r }} \in$ :. then either $\Omega_{i r} \in C_{1}$ or there does not exist a value for $\gamma_{g}$ in ? $h$.

Proof. Certainly, in view of the remark made earlier in this section and Lemma 9.1 , if there exists a value of $\gamma_{g}$ in $\leftrightarrow R$, then one of the elements of $? ?$ must lie in $C_{1}$. Since $\xi_{\omega_{l r}}>0$, we see that if $\Omega_{l r} \in \mathcal{C}^{\prime}$ and $\omega_{i r}<1$, then $\Omega_{i r} \in \mathfrak{C}^{\prime}$, by Lemma 7.1. Let $\Theta(\approx \theta) \in(?)$ such that $\Theta \in \mathcal{E}_{1}$ and $\Theta \neq \Omega_{t r}$. Let $W_{1}$ be the value in "Ill corresponding to $\Theta$, and let $W_{2}$ be the value in "ll corresponding to $\Omega_{i r}$. If $\Omega_{i r} \notin \dot{L}_{1}$. then $\omega_{i r}>1>\theta$. Let $\chi=\theta-\omega_{i r}<0$, where $\chi=\left(x_{1}+x_{2} \delta+x_{3} \delta^{2}\right) / \rho$. We have $\left|x_{2}\right|+1$ $x_{3} \delta \mid<3(3 \beta)=9 \beta$ since $\left|s_{2}\right|+\left|s_{3}\right| \delta$ and $\left|t_{2}\right|+\left|t_{3}\right| \delta$ are both less than $3 \beta$. If $\chi_{p}=\left(\xi_{\chi}, \eta_{\chi}\right)$, we have $\left|\zeta_{\chi}\right|,\left|\eta_{\chi}\right|<3$ since $\left|\eta_{\theta}\right|<1,\left|\eta_{\omega_{1},}\right|<1$ and $\left|\zeta_{\theta}\right|<1,\left|\zeta_{\omega_{1}}\right|<2$. If $X \approx \chi$, then $X \in$ ( R and $\sigma \rho|f|$ must divide $N(\rho \chi)$ by Theorem 4.2: hence

$$
|N(\chi)|=|\chi|\left(\eta_{\chi}^{2}+\zeta_{\chi}^{2}\right) \geqslant \sigma|f| / \rho^{2} .
$$

Since $\eta_{\chi}^{2}+\zeta_{\chi}^{2}<18$, we have

$$
|\chi|>\sigma|f| / 18 \rho^{2}
$$

and

$$
\begin{aligned}
I_{3} \rho|\chi| & >I_{3} \sigma|f| / 18 \rho>I_{3} / 18 \sqrt{9.0003 D} \\
& >9 \beta>\left|x_{2}\right|+\left|x_{3}\right| \delta>\left|I_{3} \rho \chi-W_{1}+W_{2}\right| .
\end{aligned}
$$

Thus, $\operatorname{sgn}(\chi)=\operatorname{sgn}\left(W_{1}-W_{2}\right)$. Since $\chi<0$ and $W_{1}-W_{2} \geqslant 0$, we have a contradiction.

Our algorithm to find a value of $\gamma_{g}$, given $\phi$ and $\psi$, can now be given as Algorithm R2 below.

Algorithm R2. Put $I_{3}>487 \beta \sqrt{D}$.

(i) Put $\tau=\rho /|f|, Q=\tau \rho$. Calculate $X_{\phi}, Y_{\phi}, X_{\psi}, Y_{\psi}$, as above.

(ii) If $\left|Y_{\psi}\right|<\rho I_{3}-\left|t_{2}\right|-\left|t_{3}\right|$, put $k=3$ and $\left(a_{1}, b_{1}\right)=(1,0),\left(a_{2}, b_{2}\right)=(0,1)$, $\left(a_{3}, b_{3}\right)=(1,-1)$. When $5\left|Y_{\psi}\right|<6 \rho I_{3}$, increase $k$ to 4 and put $\left(a_{4}, b_{4}\right)=(1,1)$. When $5\left|Y_{4}\right|>6 \rho I_{3}$, put $k=4$ and $\left(a_{1}, b_{1}\right)=(1,0),\left(a_{2}, b_{2}\right)=(1,-1),\left(a_{3}, b_{3}\right)=$ $(1,1),\left(a_{4}, b_{4}\right)=(2,1)$. 
(iii) For each pair $\left(a_{i}, b_{i}\right)(i=1,2, \ldots, k)$, calculate $Y_{i}=a_{i} Y_{\phi}+b_{i} Y_{\psi}$. If

$$
\left|Y_{i}\right|>\left[\frac{2 \sqrt{3}}{3} \rho I_{3}\right]+d_{i}+1
$$

where $d_{i}=\left|a_{1} s_{2}+b_{i} t_{2}\right|+\left|a_{i} s_{3}+b_{i} t_{3}\right|$, eliminate the corresponding pair $\left(a_{i}, b_{i}\right)$ and decrease $k$ by 1 . For the remaining pairs $\left(a_{i}, b_{i}\right)$, calculate $q_{i}=a_{i} s_{1}+b_{i} t_{1}$, $X_{i}=a_{i} X_{\phi}+b_{i} X_{\psi}$. put

$$
\begin{aligned}
& l_{11}=\left[\left(\left[X_{t} / I_{3}\right]-2 q_{i}\right) / 2 \rho\right], \\
& l_{12}=l_{11}+1 .
\end{aligned}
$$

If $\left[X_{i} / I_{3}\right] I_{3}-X_{i}>-d_{i}$ and $2 \rho \mid\left[X_{i} / I_{3}\right]-2 q_{i}$; put $u_{i}=3$ and $l_{i 3}=l_{i 1}-1$; if $\left[X_{i} / I_{3}\right] I_{3}<d_{i}-I_{3}$ and $2 \rho \mid\left[X_{l} / I_{3}\right]-2 q_{i}+1$, put $u_{i}=3$ and $l_{i 3}=l_{i 1}+2$; otherwise, put $u_{i}=2$. Compute $q_{i r}=q_{i}+\rho l_{i r}\left(1 \leqslant i \leqslant k ; 1 \leqslant r \leqslant u_{i}\right)$.

(iv) Put ${ }^{\prime l l}=\left\{I_{3} q_{i r}+X_{i} \mid 1 \leqslant i \leqslant k, 1 \leqslant r \leqslant u_{i}\right\}$, and find the minimum $\mid I_{3} q_{\kappa \lambda}+$ $X_{\kappa} \mid$ of ill. Put $m_{1}=q_{\kappa \lambda}, m_{2}=a_{\kappa} s_{2}+b_{\kappa} t_{2}, m_{3}=a_{\kappa} s_{3}+b_{\kappa} t_{3}$, and calculate the integers

$$
\begin{array}{ll}
\bar{m}_{1}=\left(m_{1}^{2}-D m_{2} m_{3}\right) /|f|, \quad \bar{m}_{2}=\left(D m_{3}^{2}-m_{1} m_{2}\right) /|f|, \\
\bar{m}_{3}=\left(m_{2}^{2}-m_{1} m_{3}\right) /|f|, \quad \Sigma=\left(m_{1} \bar{m}_{1}+D\left(m_{2} \bar{m}_{3}+m_{3} \bar{m}_{2}\right)\right) / \rho .
\end{array}
$$

If $\Sigma<0$, replace $m_{i}$ by $-m_{i}(i=1,2,3)$ and $\Sigma$ by $-\Sigma$. If

$$
\Sigma+3 \tau\left(\bar{m}_{1} \rho-m_{1} \Sigma\right) \geqslant Q^{2} .
$$

we cannot have $M \approx \mu=\left(m_{1}+m_{2} \delta+m_{3} \delta^{2}\right) / \rho$ in we eliminate $I_{3} q_{\kappa \lambda}+X_{\kappa}$ from "ll and return to the beginning of this step. If (9.2) is not true, check that

$$
\Sigma+3 \tau m_{1}<Q+3 \bar{m}_{1} .
$$

If this is not so, then $\mu>1$ and $M \notin E_{1}$. Thus, $(R$ must be a reduced lattice by Lemma 9.2. If (9.3) holds, then $M \in \mathcal{E}_{1}$, and we put $n_{1}=t_{1}, n_{2}=t_{2}, n_{3}=t_{3}$ when $\kappa=1$; otherwise, put $n_{1}=s_{1}, n_{2}=s_{2}, n_{3}=s_{3}$. We have a new basis of $\{1, \mu, \nu\}$ of , $h$, where $\nu=\left(n_{1}+n_{2} \delta+n_{3} \delta^{2}\right) / \rho$ and $M(\approx \mu) \in \mathcal{E}_{1}$. Thus, we find that $\gamma_{g}=\mu$ and $\gamma_{h}=\nu$.

With this algorithm, we are now able to conclude this section by presenting our complete reduction algorithm. This algorithm finds, from a given basis of a 1-lattice in, a reduced lattice $\varsigma$ such that $R \sim \varsigma$. It also calculates $G=-\log \gamma$, where $R=\gamma \varsigma$ and $\gamma \in \mathcal{h}$. This algorithm terminates in $O\left((\log D)^{2}\right)$ operations.

\section{Reduction Algorithm}

(i) Initialization. Put $j=1, \S_{1}=\Re, G=-\log \gamma=0$.

(ii) Find a canonical basis of $\widehat{S}_{j}$ (see Section 4 ).

(iii) Execute the pre-reduction step of Section 8 on this canonical basis of $s_{j}$. If possible, use the find step to determine a basis $\left\{1, \gamma_{g}^{(j)}, \gamma_{h}^{(j)}\right\}$ of $\delta_{j}$ and go to (v). If this is not possible, go to (iv).

(iv) Execute the algorithm $\mathrm{R} 1$ and, if necessary, $\mathrm{R} 2$.

(1) If $S_{j}$ is not reduced, then we will find a basis $\left\{1, \gamma_{g}^{(j)}, \gamma_{h}^{(j)}\right\}$ of $\delta_{j}$. After doing this, go to $(\mathrm{v})$. 
(2) If $s_{j}$ is reduced, terminate the reduction algorithm. We have $s=s_{j}$ and $G=-\log \gamma$.

(v) Perform the invert step of Section 4 on the basis $\left\{1, \gamma_{g}^{(j)}, \gamma_{h}^{(j)}\right\}$ of $\bar{s}_{j}$ to find a basis of the lattice $s_{j+1}=\left|\gamma_{g}^{(j)}\right|^{-1} s_{j}$. Replace $G$ by $G+\log \left(1 /\left|\gamma_{g}^{(j)}\right|\right)$, increase $j$ by 1 and return to (ii).

10. Implementation and Computational Results. We call the process by which we obtain a basis $\left\{1, \theta_{g}^{(r+1)}, \theta_{h}^{(r+1)}\right\}$ of $\left(h_{r+1}\right.$ from a basis $\left\{1, \theta_{g}^{(r)}, \theta_{h}^{(r)}\right\}$ of $h_{r}$, (see Section 3) a simple step. One means of doing this has been fully described in [18]. When this algorithm was implemented for values of $D>10^{6}$ it was found that the amount of precision needed by Algorithm II of [18] was large enough to slow the program's running time significantly. To overcome this difficulty we modified Algorithm II along the lines of Algorithm R2 of Section 9.

We replaced step (ii) of Algorithm II by the step

(ii') If $\left|Y_{\psi}\right|<\sigma_{r} I_{3}-\left|t_{2}\right|-\left|t_{3}\right|$, put $k=3$ and $\left(a_{1}, b_{1}\right)=(1,0),\left(a_{2}, b_{2}\right)=(0,1)$, $\left(a_{3}, b_{3}\right)=(1,-1)$. When $\left|Y_{\psi}\right| \geqslant \sigma I_{3}-\left|t_{2}\right|-\left|t_{3}\right|$ and $4\left|X_{\psi}\right|>\sigma_{r} I_{3}$, put $k=4$ and $\left(a_{1}, b_{1}\right)=(1,0),\left(a_{2}, b_{2}\right)=(0,1)\left(a_{3}, b_{3}\right)=(1,-1),\left(a_{4}, b_{4}\right)=(1,1)$. When $\left|Y_{\psi}\right| \geqslant$ $\sigma I_{3}-\left|t_{2}\right|-\left|t_{3}\right|$ and $4\left|X_{4}\right|<\sigma_{r} I_{3}$, put $k=4$ and $\left(a_{1}, b_{1}\right)=(1,0), \quad\left(a_{2}, b_{2}\right)=$ $(1,-1),\left(a_{3}, b_{3}\right)=(1,1),\left(a_{4}, b_{4}\right)=(2,1)$. Let $\mathbb{H}^{\prime}=\left\{\left(a_{i}, b_{i}\right) \mid 1 \leqslant i \leqslant k\right\}$.

We also replaced step (iii) by the step

(iii') For each pair $\left(a_{i}, b_{i}\right) \in\left(\mathcal{A}^{\prime}\right.$ calculate $Y_{i}=a_{i} Y_{\phi}+b_{i} Y_{\psi}, d_{i}=\left|a_{i} s_{2}+b_{i} t_{2}\right|+$ $\left|a_{i} s_{3}+b_{i} t_{3}\right|$. If

$$
\left|Y_{i}\right|>\left[\frac{2 \sqrt{3}}{3} \sigma_{r} I_{3}\right]+d_{i}+1,
$$

eliminate the corresponding pair $\left(a_{i}, b_{i}\right)$ from $\Theta$ and decrease $k$ by 1 . For the remaining pairs in $Q$ calculate $q_{i}=a_{i} s_{1}+b_{i} t_{1}, X_{i}=a_{i} X_{\phi}+b_{i} Y_{\psi}$. Put

$$
l_{i 1}=\left[\left(\left[X_{i} / I_{3}\right]-2 q_{i}\right) / 2 \sigma_{r}\right], \quad l_{i 2}=l_{i 1}+1 .
$$

If $\left[X_{i} / I_{3}\right] I_{3}-X_{i}>-d_{i}$ and $2 \sigma_{r} \mid\left[X_{i} / I_{3}\right]-2 q_{i}$, put $u_{i}=3$ and $l_{i 3}=l_{i 1}-1$; if

$$
\left[X_{i} / I_{3}\right] I_{3}-X_{i}<d_{i}-I_{3} \text { and } 2 \sigma_{r} \mid\left[X_{i} / I_{3}\right]-2 q_{i}+1 \text {, }
$$

put $u_{i}=3$ and $l_{i 3}=l_{i 1}+2$; otherwise, put $u_{i}=2$. Compute $q_{i j}=q_{i}+\sigma_{r} l_{i j}(1 \leqslant i$ $\left.\leqslant k, 1 \leqslant j \leqslant u_{i}\right)$ and $\mathscr{W}=\left\{I_{3} q_{i j}+X_{i} \mid 1 \leqslant i \leqslant k, 1 \leqslant j \leqslant u_{i}\right\}$.

This new version of Algorithm II is valid for $I_{3}>18^{2} \sqrt{3} \delta^{3}$, a much smaller value for $I_{3}$ than that needed in the old version. This follows from the methods used in Section 9 here, especially Lemma 9.1. We must also make use of the methods of Lemma 9.2 of this work, Lemma 9.3 of [18] and (8.3).

Let $\mathcal{R}_{r}$ have $\left\{1,\left(m_{1}+m_{2} \delta+m_{3} \delta^{2}\right) / \sigma_{r},\left(n_{1}+n_{2} \delta+n_{3} \delta^{2}\right) / \sigma_{r}\right\}$ as a basis, and suppose $R_{r}=\sum_{i=1}^{r-1} \log \theta_{g}^{(i)}$. The complete algorithm for a doubling step when $\delta^{3}=D$ is given below.

\section{Doubling Algorithm}

(i) Find a canonical basis of $\mathcal{R}_{r}$ as described in Section 4.

(ii) Find a basis of $\mathcal{R}_{r}^{2}$ by using the method of Theorem 6.2. Also, calculate $w$ by the corollary of Theorem 6.2 . 
(iii) Apply the reduction algorithm of Section 9 to $\mathcal{R}_{r}^{2}$ to find a basis $\{1, \mu, \nu\}$ of a reduced lattice $S\left(\sim \Omega_{r}^{2}\right)$, together with $G=-\log \gamma$, where $\gamma \delta=\mathcal{R}_{r}^{2}$. Compute $\bar{R}_{r}=2 R_{r}-G-\log w$.

After we have performed this algorithm, we have a new reduced lattice $S=\Omega_{m}=$ $\langle 1, \mu, \nu\rangle$ such that $m \simeq 2 r$. Further $R_{m}=\bar{R}_{r}$.

We will also require an algorithm which, given some lattice $Q_{R}$ and another lattice ${ }^{\prime} R_{k}$, finds a lattice $R^{*}=Q R_{n}$, with $R^{*}=R_{n}$, where $n \simeq k+r$. We call this the large step algorithm. We first put $P_{i}=\sigma_{i} / \operatorname{gcd}\left(\sigma, e_{i}\right)$; this is $L\left(a_{i}\right)$ for the ideal $a_{i}$ corresponding to $R_{i}$.

\section{Large Step Algorithm}

(i) Find $\Omega_{j}$ such that $\operatorname{gcd}\left(P_{r}, P_{j}\right)=1$ and $R_{j} \leqslant R_{k}$.

(ii) Find canonical bases for $\ell_{R}$, and $\ell_{R}$.

(iii) Use formulas (5.15) to find a basis of $R_{R}=\mathscr{R}_{j} \Re_{r}$.

(iv) Apply the reduction algorithm of Section 9 to $\Omega$ to find a basis of a reduced lattice $\ell^{*}(\sim Q R)$ together with $G=-\log \gamma$, where $\gamma^{\Re *}=\Re$. Since $\operatorname{gcd}\left(L\left(a_{i}\right), L\left(a_{j}\right)\right)=1$, we have $t=1$ in (3.5) and $R^{*}=R_{r}+R_{j}-G$. (Note that $R^{*} \leqslant R_{r}+R_{j}$.)

Remark. In order to accomplish step (i), it is often convenient to have a pre-computed list of lattices $\Re_{k-i}(i=1,2,3, \ldots, m)$. Since the probability that two randomly selected integers be relatively prime is $6 / \pi^{2}, m$ need not be very large. Of course, one could dispense entirely with this search step by using the formulas in Section 5 to obtain a general composition algorithm for finding $\Re_{k} \Re_{r}$. Such an algorithm would execute in $O(\log D)$ operations. However, in practice, the search for ${ }_{R},{ }_{j}$ here is usually very brief. In fact, we found that most often $j=k$ or $k-1$.

We will also need the following simple lemma.

LEMMA 10.1. Suppose we are given t lattices

$$
Q_{h}, Q_{R}, Q_{2} \Omega_{3 b}, \ldots, R_{t b},
$$

together with the values of $R_{b}, R_{2 b}, R_{3 b}, \ldots, R_{t b}$. Suppose further that $R_{t b} \leqslant R$ and $\bar{\Omega}$ is a lattice such that $\bar{R}=m R$, where $m \in \mathcal{Z}$ and $m \geqslant 1$. If $R_{r}<\bar{R} \leqslant R_{n}$, where $R_{n}-R_{r} \leqslant R_{b(t-1)}$, then one of the lattices

$$
\Re_{n}, \Re_{n+1}, \Re_{n+2}, \ldots, \Re_{n+b-1}
$$

must be one of the lattices in (10.1).

Proof. Let $\theta_{n}=\varepsilon_{0}^{q_{1}} \theta_{u}, \theta_{r}=\varepsilon_{0}^{q_{2}} \theta_{v}$, where $1 \leqslant \theta_{u}, \theta_{v}<\varepsilon_{0}$. Then $\Re_{u}=\Re_{n}, \Re_{v}=\mathscr{R}_{r}$ and $R_{u}, R_{v}<R$. Since $R_{n}-R_{r}<R$, we get

$$
0<R_{r}-(m-1) R<R \leqslant R_{n}-(m-1) R<2 R \text {. }
$$

Thus,

$$
R_{v}=R_{r}-(m-1) R, \quad R_{u}=R_{n}-m R \text { and } R_{u}+R-R_{v} \leqslant R_{b(t-1)} .
$$

Since $R-R_{v}>0$, we have $R_{u}<R_{t(b-1)}$. It follows that one of the $b$ lattices $\Re_{n}, \Re_{n+1}, \Re_{n+2}, \ldots, \Re_{n+b-1}$ must be one of the lattices in (10.1). 
By using this lemma we can develop the search algorithm. We assume that the sequence of lattices $(10.1)$ has been pre-computed and that $\left(R_{h}=(h)(t-1) r\right.$. Given a lattice $R_{r}$ and an upper bound $B$, this algorithm finds (if it exists) the value of $\bar{R}$ for a lattice $\frac{r}{R}$ such that

$$
R_{r}<\bar{R} \leqslant B \quad \text { and } \quad \bar{R}=m R \quad(m \in \text { : } m \geqslant 1) .
$$

It does this by searching the range between $R_{r}$ and $B$ in large steps of size $R_{h}$.

The Search Algorithm

(i) Use $R_{r}$ and $h_{h}$ with the large step algorithm above to find $h^{*}$ and $R^{*}$.

(ii) Assuming $?_{n}=\left(R^{*}\right.$, perform simple steps on $R_{n}$ to find

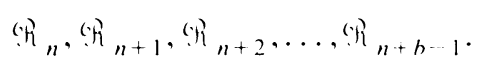

(iii) Compare each of the lattices of (10.2) with the $t$ lattices of (10.1). This is most rapidly accomplished if the $\sigma_{h i}$ values for the lattices in (10.1) have been pre-sorted. If any $\sigma_{h i}=\sigma_{n+j}$, compare $\left|e_{h i}\right|$ and $\left|e_{n+j}\right|$. If they are not equal, we do not have a match; if they are equal, then compare $h_{h i}$ and $h_{n+j}$ by finding a canonical basis for both and comparing coefficients. (If the search routine is to be used frequently, it is a good idea to pre-compute canonical bases for the lattices in (10.1).) If we get a match $?_{h i}=R_{n+j}$, then $\bar{R}=R_{n+j}-R_{b i}$, and we terminate the algorithm. If we do not find a match, go to (iv).

(iv) If $B \leqslant R^{*}$, the algorithm terminates, and we know that $\bar{R}$ does not exist. If $B>R^{*}$, put $\ell_{r}=R^{*}, R_{r}=R^{*}$ and return to (i).

If we know in advance that there are two elements $\Theta_{i}\left(\approx \theta_{i}\right), \Theta_{j}\left(\approx \theta_{j}\right)$ in the chain (3.1) such that $\theta_{i}<\theta_{j}<\varepsilon_{0}$ and $N\left(\theta_{i}\right)\left|J^{2}, N\left(\theta_{j}\right)\right| J^{2}$ (see [15], [16] for some simple criteria), put $p=1$; otherwise, put $p=0$. When $p=1$, we have $\varepsilon_{0}=\theta_{i}^{3} / N\left(\theta_{i}\right)$ and $R=3 R_{i}-\log N\left(\theta_{i}\right)$.

We are now ready to give the algorithm for finding $R$ and $h$. We assume that $E$ $=\sqrt{3} c_{2} J F(Q) / 2 \pi$ has been pre-computed and that we have values for input parameters, $D, E, L, b, t, x, k$.

\section{The Main Algorithm}

(i) By using simple steps starting from $h$, calculate and store in memory a table of lattices each of which is represented by the set of integers

$$
\left\{m_{1}^{(i)}, m_{2}^{(i)}, m_{3}^{(i)}, n_{1}^{(i)}, n_{2}^{(i)}, n_{3}^{(i)}, \sigma_{i}\right\},
$$

where the corresponding lattice ${ }^{R} R_{i}$, has basis

$$
\left\{1,\left(m_{1}^{(i)}+m_{2}^{(i)} \delta+m_{3}^{(i)} \delta^{2}\right) / \sigma_{i},\left(n_{1}^{(i)}+n_{2}^{(i)} \delta+n_{3}^{(i)} \delta^{2}\right) / \sigma_{i}\right\}
$$

We also store the corresponding value of $R_{i}$. The lattices we store are ${ }^{2}{ }_{j h}$ $(j=1,2,3, \ldots, t)$. It is also convenient when performing large steps to have a table of lattices ${ }^{\prime} R_{t b-j}(j=0,1,2,3, \ldots, 2 b)$. If in the process of creating these tables, we find $R$, that is, $R \leqslant 3 R_{b t}-\log J^{2}$ when $p=1$ or $R \leqslant R_{b t}$ when $p=0$, then we can usually calculate $h$ from $E$ without much trouble (see [1]), and we are done. We may assume that $R_{b t}<R$ for the rest of this algorithm.

(ii) Put $L_{1}=R_{b t}$, and find that value of $\kappa$ such that $E / 2^{\kappa}<L_{1}$ and $E / 2^{\kappa-1}>L_{1}$. Put $U=E / 2^{\kappa}$, and find in the table produced in step (i) that lattice $R_{i u}$ such that 
$R_{i u}<U$ and $R_{i(u+1)}>U$. Perform simple steps starting with ${ }^{\circ} R_{i u}$ to find that lattice ( $R{ }_{n}$ such that $R_{n}<U$ and $R_{n+1}>U$. Put $\lambda=1$.

(iii) Perform the Doubling step on $R_{n}$ to produce $R_{m}$ and $R_{m}$. Perform simple steps, starting with $?_{m}$ to obtain $\leftrightarrow_{h}$ and $R_{h}$, where $R_{h}<2^{\lambda} U$ and $R_{h+1}>2^{\lambda} U$. Increment $\lambda$ by 1 , and replace $R_{n}$ and $R_{n}$ by $\Omega_{h}$ and $R_{h}$, respectively, If $\lambda \leqslant \kappa$, return to the beginning of step (iii); otherwise go to (iv).

(iv) We now have a lattice ${ }^{h}{ }_{n}$ where $R_{n} \simeq E$. Use the search algorithm to find $R$ starting at $R_{n}$ and going up to $B=E+L$. If this is unsuccessful, replace $E$ by $E-L$ and go to (ii). If this fails to find $\bar{R}$, we say that the algorithm fails and we either increase $L$ or terminate the algorithm; otherwise, we now have $\bar{R}=h R$. Put $E=\bar{R}$.

(v) We now determine $h$. We first put $i=h^{*}=1, H=E$.

(vi) Find $v$ and all primes $q_{a}(a=1,2,3, \ldots, v)$ such that $q_{1}=2, q_{a}>q_{a-1}$, and $q_{a}<\sqrt{E / k L_{1}}(a=1,2,3, \ldots, v)$.

(vii) If $i \leqslant v$ go to (1); otherwise, go to (2).

(1) By using the methods of (ii) and (iii), find $R_{n}$ and $R_{n}$ such that $R_{n}<E / q_{i}-x$ and $R_{n+1}>E / q_{i}-x$. That is, we use $E / q_{i}-x$ for $E$ in (ii) and (iii) in order to find $\ell_{n}$. Perform simple steps starting with $Q_{n}$ until we find some $\Omega_{k}$ such that $\sigma_{k}=1$ or $R_{k}>E / q_{i}+x$. If the former case occurs, we know that $q_{i} \mid h$. We replace the value of $h^{*}$ by that of $q_{i} h^{*}$, the value of $E$ by that of $E / q_{i}$ and return to (vi). If the latter case occurs, we know that $q_{i} \nmid h$. We increment $i$ by 1 , and we return to the beginning of step (vii).

(2) Starting with $\Re_{r}=Q_{h}$, use the search algorithm to find $\bar{R}$ with $B$ $=\sqrt{k L_{1} E}+x$.

(viii) If, in executing (2) we find $\bar{R}$, then $R=\bar{R}$ and $h=h^{*}(E / R)$. If we do not find $\bar{R}$, then $R>\sqrt{k L_{1} E}$. Hence, $h / h^{*}<E / \sqrt{k L_{1} E}=\sqrt{E / k L_{1}}$. Thus, if $q \mid\left(h / h^{*}\right)$, then $q<\sqrt{E / k L_{1}}$ and, since all such $q$ 's have been examined, it follows that $h=h^{*}$ and $R=H / h$.

Remarks. (1) We could have stored all of the lattices computed in step (i) instead of $1 / b$ of them; but, if we wish $L_{1}$ to be large, this may require more storage than the computer is capable of handling.

(2) We make use of $x$ in substeps (vii)-1, and (vii)-2 in order to ensure that we do not skip over any lattices that should be examined. This could happen because of round off or truncation errors in the evaluation of $R_{n}$ or $\bar{R}$. For example, the computer's estimate of $R_{n}$ might be less than $E$ or $E / q_{i}$, but the actual value of $R_{n}$ might not be. The value of $x$ need not be very large but must be large enough to overcome this possibility.

(3) It is difficult to find what value to use for $L$ here. We give below a theoretic estimate which requires the truth of a certain Generalized Riemann Hypothesis (GRH). However, a glance at the results in Table 3 will show that this estimate for $L$ will often be much larger than that needed in practice. In the range of values of $D$ used here we found that $L$ between 50000 and 300000 would usually work.

(4) Because we cannot be sure of how good our approximation $F(Q)$ is to $\Phi(1)$, we cannot prove with full mathematical rigour that the value we get here for $h$ is correct. From the results given below we would expect that $F(Q)$ should give a reasonable approximation to $\Phi(1)$ when $Q$ is fairly large. Hence, when $h$ is small, our method is 
very likely to find it correctly. In spite of our slight lack of confidence in $h$, however, the value that we obtain for $R$ is correct. This is simply because we get a value of $\bar{h} R$ in step (iv), and then we find $\bar{h}$ in step (viii). Nowhere in the process of finding $\bar{h}$ did we really have to assume that it was actually $h$.

As indicated in Remark (3) above, a problem in running this algorithm is knowing what value to give $L$. We now discuss a theoretic estimate of a value for $L$ which ensures that $\bar{R}$ will be found. This requires an analysis of how good an approximation $E$ is to $h R$. Of course we will find $\bar{R}$ when

$$
L>|E-h R| \text {. }
$$

Let $\Pi_{x}^{\prime}\left(\Sigma_{x}^{\prime}\right)$ represent the product (sum) over all primes $q$ such that $q>x, q \equiv 1$ $(\bmod 3)$ and $q \nmid J$. Let $Q>3$, and put $T(Q)=\Pi_{Q}^{\prime} f(q)$. Then

$$
\Phi(1)=c_{2} F(Q) T(Q)
$$

and

$$
|E-h R|<c_{2} \sqrt{3} J F(Q)|1-T(Q)| / 2 \pi
$$

In order to estimate $T(Q)$, we will use a method very similar to that employed by Cornell and Washington [5].

We have

$$
T(Q)=\prod_{Q}^{\prime} q^{2} /\left(q^{2}+\varepsilon(q) q+1\right)
$$

where

$$
\varepsilon(q)= \begin{cases}-2 & \text { when }(D / q)_{3}=1 \\ 1 & \text { when }(D / q)_{3} \neq 1\end{cases}
$$

Hence,

$$
\log T(Q)=-\sum_{Q}^{\prime} \varepsilon(q) / q+\gamma
$$

where $|\gamma|<3 / Q$. Put $B(Q)=\Sigma_{Q}^{\prime} \varepsilon(q) / q$ and $A(t)=\Sigma_{q \leqslant t} \varepsilon(q)$. We now require the effective version of the Chebotarev Density Theorem of Lagarias and Odlyzko [7] with constants given by Oesterle [10]. This requires further that we assume the truth of the GRH for the zeta function $\zeta_{\xi}$, where $\xi=2(\sqrt[3]{D}, \rho)$ and $\rho^{2}+\rho+1=0$.

We note that $n_{\varepsilon}=[\varepsilon: 2]=6$ and $d_{\varepsilon}$, the discriminant of $\varepsilon$, is $27 J^{4}$. Further, if $G$ is the Galois group of $\mathcal{E}$, then $|G|=6$. Now $A(t)=-2 \pi_{C_{1}}(t)+\pi_{C_{2}}(t)$, where the symbols $C$ and $\pi_{C}$ are defined in [10]; also, $\left|C_{1}\right|=1,\left|C_{2}\right|=2$. Thus, by [10]

$$
A(t)=-2 \pi_{C_{1}}(t)+\frac{2\left|C_{1}\right|}{|G|} \operatorname{Li}(t)+\pi_{C_{2}}(t)-\frac{\left|C_{2}\right|}{|G|} \operatorname{Li}(t)
$$

and

$$
\begin{aligned}
|A(t)| & \leqslant \frac{2}{3} \sqrt{t}\left[\left(\frac{1}{\pi}+\frac{5.3}{\log t}\right) \log 27 J^{4}+6\left(\frac{\log t}{2 \pi}+2\right)\right] \\
& =\sqrt{t}(\log t) C(t)
\end{aligned}
$$


where $C(t)$ is a monotone decreasing function of $t$. By using the reasoning of [5] we find that

$$
|B(Q)|<\left(\frac{4+3 \log Q}{\bar{Q}}\right) C(Q) .
$$

When $Q^{5}>\max \left(10^{30}, 27 J^{4}\right), J>10^{6}$, then

$$
|1-T(Q)|<\frac{6 \log \left(27 J^{4} Q\right)}{\bar{Q}}
$$

and

$$
|E-h R|<\frac{3 \sqrt{3} c_{2}}{\pi_{\downarrow} \bar{Q}} J \log \left(27 J^{4} Q\right) F(Q) .
$$

Since evaluating $E$ requires $O(Q)$ operations and, by Mertens' Theorem,

$$
F(Q)<\prod_{q \leqslant Q}\left(\frac{q}{q-1}\right)^{2}=\left[\prod_{q \leqslant Q}(1-1 / q)\right]^{-2} \sim\left(e^{\gamma} \log Q\right)^{2},
$$

we see that if $L_{1} \simeq D^{2 / 5}$, then in order to evaluate $E$ and $\bar{R}$ we require (putting $\left.Q=D^{2 / 5}\right) O\left(D^{2 / 5+\varepsilon}\right)$ operations. Further, since $\sqrt{k E L_{1}} / L_{1}=O\left(D^{3 / 10+\varepsilon}\right)$, we see that we require $O\left(D^{2 / 5+\varepsilon}\right)$ operations to find $R$ and $h$ by the above algorithm under the GRH for $\zeta_{\text {s. }}$.

Let $r$ be the number of steps required by Voronoi's algorithm to find $R$. Since $R=\Sigma_{1}^{r} \log \theta_{g}^{(i)}$ and $1<\theta_{g}^{(i)}<1+\xi_{\theta}$, where $\left(\xi_{\theta}, \eta_{\theta}\right)$ is the puncture of $\Theta_{g}^{(i)}$, we have $R<r \log (1+(3 \sqrt{3}+3) D)$ by formula 6.8 of [18]. In fact, it seems by empirical results that $R \simeq 1.12 r$. From the results developed above, we see that we have $R h=O\left(D^{1-\varepsilon}\right)$. Thus, we expect Voronoi's algorithm to take about $O(D / h)$ steps to find $R$. Even if we do not assume the GRH, we can use the well-known result that $\Phi(1)=O\left((\log |\Delta|)^{1+\varepsilon}\right)$. With this we can show that our ideas will provide a method which executes in $O\left(D^{1 / 2+\varepsilon}\right)$ operations. Thus, if $h$ is small our new method is faster than that which uses a straight application of Voronoi's algorithm.

The algorithm described above was implemented in FORTRAN- $H$ (extended) for an AMDAHL 470-V7 computer. The extended precision feature allowed us to operate on numbers of up to 33 decimal digits. For values of $D<2^{31}$, this amount of precision is sufficient except for the find step and the invert step. For these steps we obtained more precision by using special purpose multi-precision assembler language subroutines. This program was usually run with $L=50000, b=15, k=10, t=1500$. On running the program, we found that the amount of time taken to perform a simple step was about 4.3 milliseconds. The time required to perform a doubling step or a large step was usually between 100 and 400 milliseconds, the average time being 250 milliseconds. Most of this time was consumed by the multi-precision find and invert sections of the reduction algorithm. In Table 3 below, we give some results of running our program for large values of $D$. These values were selected with an eye to obtaining large values of $R$; hence, the values of $D$ are primes congruent to 2 or $5(\bmod 9)$ such that $\Phi(1)$ is large. 
TABLE: 3

D

E

$h R$

R

$\mathrm{h}$

$\begin{array}{rr}1079021 & 2734906.136341 \\ 2609069 & 7400272.677097 \\ 2961713 & 7665950.516997 \\ 3650249 & 9782563.073006 \\ 4248551 & 11409669.209399 \\ 7191911 & 19018244.808396 \\ 8688161 & 23904403.372750 \\ 9488513 & 26068028.870131 \\ 14613773 & 39788531.398671 \\ 16477553 & 43721343.379590 \\ 17353643 & 44095839.633036 \\ 18209801 & 52783288.770296 \\ 19196813 & 52633382.613328 \\ 19446881 & 51387164.594867 \\ 19914539 & 54729047.586607 \\ 20281169 & 53486260.159788 \\ 21745121 & 57252172.105084 \\ 23301797 & 61614171.225781 \\ 23444777 & 61469132.178084 \\ 20006741 & 46521260.090157 \\ 2000003749\end{array}$

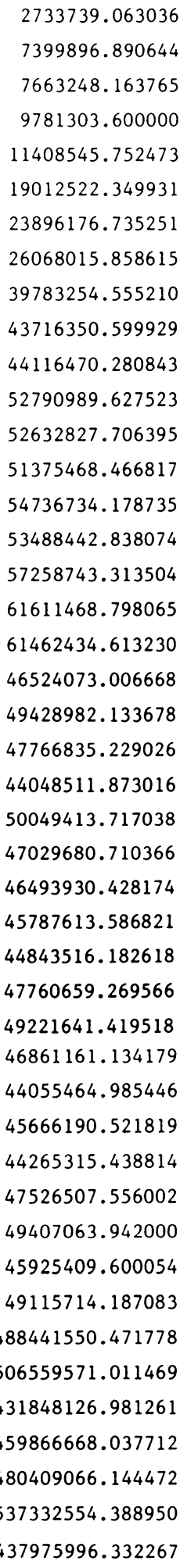

341717.382879

3699948.445321

7663248.163765

4890651.800000

2852136.438118

9506261.174966

5974044.183831

2606801.585862

4972906.819401

63541.207267

8823294.056169

13197747.406881

26316413.853197

51375468.446817

5473673.417873

53488442.838074

57258743.313504

12322293.759613

61462434.613230

46524073.006668

24714491.066839

23883417.614513

3146322.276644

25024706.858519

47029680.710366

46493930.428174

22893806.793410

44843516.182618

47760569.269566

12305410.354880

5857645.141772

22027732.492723

22833095.260910

44265315.438814

4752650.755600

24703531.971000

22962704.800027

49115714.187083

122110387.617945

253279785.505735

431848126.981261

459866668.037712

240204533.072236

134333138.597237

437975996.332267
8

2

1

2

4

2

4

10

8

688

5

4

2

1

10

1

1

5

1

1

2

2

14

2

1

1

2

1

1

4

8

2

2

1

10

2

2

1

4

2

1

1

2

4

1 
TABLE 3 (continued)

\begin{tabular}{|c|c|c|c|c|}
\hline D & E & $\mathrm{hR}$ & $\mathbf{R}$ & $\mathrm{h}$ \\
\hline 200099381 & 497603952.415591 & 497590195.640789 & 124397548.910197 & 4 \\
\hline 200100389 & 424196870.367996 & 424203442.573298 & 424203442.573298 & 1 \\
\hline 200100503 & 451182094.347744 & 451197215.352740 & 451197215.352740 & 1 \\
\hline 200107571 & 480433899.513446 & 480444645.440266 & 480444645.440266 & 1 \\
\hline 200112401 & 472253194.115455 & 472167872.629630 & 118041968.157408 & 4 \\
\hline 200114699 & 404492099.980046 & 404488912.856795 & 202244456.428397 & 2 \\
\hline 200122889 & 464342463.344755 & 464299134.881723 & 58037391.860215 & 8 \\
\hline 200164271 & 441701163.856313 & 441705327.026624 & 220852663.513312 & 2 \\
\hline 200171999 & 518597740.739617 & 518594546.969083 & 518594546.969083 & 1 \\
\hline 200182403 & 440166276.950028 & 440210372.576431 & 220105186.288216 & 2 \\
\hline 200182529 & 447398884.585019 & 447390972.406384 & 22369548.620319 & 20 \\
\hline 200237591 & 458593160.418426 & 458631734.208582 & 229315867.104291 & 2 \\
\hline 1000002821 & 2224268272.85790 & 2224244048.137217 & 2224244048.137217 & 1 \\
\hline 1000021079 & 2415725386.65008 & 2415802816.494235 & 603950704.123559 & 4 \\
\hline 1000022213 & 2176263035.09785 & 2176209325.109052 & 272026165.638631 & 8 \\
\hline 1000027001 & 2378191472.68543 & 2378124348.205241 & 1189062174.102620 & 2 \\
\hline 1000050017 & 2131249352.94466 & 2131259056.681105 & 2131259056.681105 & 1 \\
\hline 1000069643 & 2178195190.70341 & 2178254057.347236 & 544563514.336809 & 4 \\
\hline 1000091399 & 2235417592.91327 & 2235363727.828276 & 1117681863.914138 & 2 \\
\hline 1000115579 & 2340780764.18363 & 2340733780.230313 & 37753770.648876 & 62 \\
\hline 2000001359 & 5097379160.99403 & 5097475496.226634 & 1274368874.056659 & 4 \\
\hline 2000009129 & 4675237416.61298 & 4675293317.830901 & 4675293317.830901 & 1 \\
\hline 2000012477 & 4148134404.14319 & 4148034373.232793 & 4148034373.232793 & 1 \\
\hline 2000029403 & 4826616791.70067 & 4826399013.912892 & 2413199506.956446 & 2 \\
\hline 2000052137 & 4983495105.51258 & 4983602513.601523 & 4983602513.601523 & 1 \\
\hline 2000108111 & 4605978642.80285 & 4606048408.531975 & 1151512102.132994 & 4 \\
\hline 2000131223 & 4840963289.88280 & 4840696491.272803 & 121017412.281820 & 40 \\
\hline 2000145629 & 4937983529.32301 & 4937740516.447692 & 4937740516.447692 & 1 \\
\hline
\end{tabular}

For $D=2000145629$, we found the value of $h R$ in 3.6 minutes of CPU time. To test the primes up to 151 as possible divisors of $h$ required 1.8 minutes, and a further 8.6 minutes was needed for the search routine in step (vii)- 2 to execute. The total time required to find $R$ and $h$ was 14 minutes. Probably these times could be reduced by careful tuning of the values of the input parameters $b, t, k$. To find $R$ using Voronoi's algorithm alone would probably require about $(1 / 3)\left(4.3 \times 10^{-3}\right) R / 1.12$ seconds or 73 CPU days.

11. Acknowledgements. The authors gratefully acknowledge suggestions of $\mathrm{H}$. W. Lenstra, Jr., and R. Schoof for improving the speed of this algorithm. These suggestions led to the development of the search algorithm. They also wish to thank Daniel Shanks for originally suggesting this problem and for his continued interest and encouragement.

Department of Computer Science

University of Manitoba

Winnipeg, Manitoba, Canada R3T 2N2 
1. P. Barrucand, H. C. Williams \& L. Baniuk, "A computational technique for determining the class number of a pure cubic field," Math. Comp., v. 30, 1976, pp. 312-323.

2. W. E. H. BERwick, "The classification of ideal numbers that depend on a cubic irrationality." Proc. London Math. Soc., v. 12, 1913, pp. 393-429.

3. W. E. H. BerWICK, "The arithmetic of quadratic number fields," Math. Gazette, v. 14, 1928, pp. $1-11$.

4. J. W. S. CASSELS, "The rational solutions of the diophantine equation $Y^{2}=X^{3}-D$," Acta Math.. v. 82,1950, pp. $243-273$.

5. G. Cornell \& L. Washington, "Class numbers of cyclotomic fields," J. Number Theory. (To appear.)

6. B. N. Delone \& D. K. FaddeEv, The Theory of Irrationalities of the Third Degree. Transl. Math. Mono., vol. 10, Amer. Math. Soc.. Providence, R.1., 1964.

7. J. C. Lagarias \& A. M. Odlyzko. "Effective versions of the Chebotarev density theorem." Algebraic Number Fields, Academic Press, New York, 1977, pp. 409-464.

8. E. LANDAU, Vorlesungen über Zahlentheorie, Vol. II, Chelsea, New York. 1955.

9. H. W. Lenstra, JR., On the Calculation of Regulators and Class Numbers of Quadratic Fields. Report 80-08, Mathematisch Instituut, Amsterdam, 1980.

10. J. Oesterle, "Versions effectives du théoreme de Chebotarev sous l'hypothése de Riemann généralisée," Astérisque, v. 61, 1979, pp. 165-167.

11. R. J. Schoof, Quadratic fields and factorization, Studic Week Getaltheorie en Computers, Math. Centrum, Amsterdam, 1980, pp. 165-206.

12. D. Shanks, The Infrastructure of a Real Quadratic Field and its Applications. Proc. 1972 Number Theory Conference, Boulder, 1972, pp. 217-224.

13. D. Shanks, "A survey of quadratic, cubic and quartic algebraic number ficlds (from a computational point of view)," Congressus Numerantium, v. 17, 1976, pp. 15-40.

14. G. F. Voronor, Concerning Algebraic Integers Dericable from a Root of an Eiquation of the Third Degree, Master's Thesis, St. Petersburg, 1894. (Russian)

15. G. F. Voronol, On a Generalization of the Algorithm of Continued Fractions, Doctoral Disscrtation. Warsaw, 1896. (Russian)

16. H. C. Williams, "Improving the speed of calculating the regulator of certain pure cubic ficlds." Math. Comp., v. 34, 1980, pp. 1423-1434.

17. H. C. Williams, "Some results concerning Voronoi's Continued Fraction over $Q(\sqrt[3]{D})$," Math. Comp., v. 36, 1981, pp. 631-652.

18. H. C. Williams, (;. Cormack \& E. Seah, "Calculation of the regulator of a pure cubic field." Math. Comp., v. 34, 1980, pp. 567-611.

19. H. C. Williams \& D. Shanks, “A note on class-number one in pure cubic ficlds," Math. Comp.. v. 33, 1979, pp. 1317-1320. 